\title{
The Asymmetric Piers Hydrosilylation
}

Lars Süsse, Julia Hermeke, and Martin Oestreich*

Institut für Chemie, Technische Universität Berlin,

Straße des 17. Juni 115, D-10623 Berlin, Germany

martin.oestreich@tu-berlin.de

\section{Supporting Information}

Table of Contents

1 General Information

S2

$2 \quad$ General Procedures (GPs)

2.1 GP 1: General Procedure for the Optimization of the Borane-Catalyzed S4 Hydrosilylation of Acetophenone (6)

2.2 GP 2: General Procedure for Borane-Catalyzed Hydrosilylation of Ketones S4

2.3 GP 3: General Procedure for Preparation of Racemic Alcohols

3 Experimental Details for the Preparation of Borane Adducts (S)-3.3,3-DMO and (S)-3.DMS

3.1 (S)-4-(Pentafluorophenyl)-4,5-dihydro-3H-(3,3'-diphenyl)dinaphtho[2,1-c:1',2'-e] S5 borepine $[(S)-3]$

3.2 (S)-4-(Pentafluorophenyl)-4,5-dihydro-3H-(3,3'-diphenyl)dinaphtho[2,1-c:1',2'-e] S6 borepine 3,3-Dimethyloxetane Adduct [(S)-3·3,3-DMO]

3.3 (S)-4-(Pentafluorophenyl)-4,5-dihydro-3H-(3,3'-diphenyl)dinaphtho[2,1-c:1',2'-e] S7 borepine Dimethyl Sulfide Adduct [(S)-3·DMS]

4 Optimization of the Borane-Catalyzed Hydrosilylation of Acetophenone S10

$5 \quad$ Procedures and Characterization Data for Alcohols 7 and 23-37 S12

$6 \quad$ NMR Spectra of All Compounds $\quad$ S24

$7 \quad$ HPLC Traces of Alcohols 7 and 23-37 S73

8 References $\quad$ S89 


\section{General Information}

All reactions were performed in flame-dried glassware using an MBraun glove box $\left(\mathrm{O}_{2}<10 \mathrm{ppm}, \mathrm{H}_{2} \mathrm{O}<0.5 \mathrm{ppm}\right)$ or conventional Schlenk techniques under a static pressure of argon (glove box) or nitrogen. Liquids and solutions were transferred with syringes. 1,2-Diflourobenzene was purified and dried following a standard procedure. $\mathrm{CH}_{2} \mathrm{Cl}_{2}$ and $n$-hexane were purified and dried using an MBraun solvent system. Technical grade solvents for chromatography (n-pentane and ethyl acetate) were distilled prior to use. Dihydrostannepine $(S)-4,{ }^{[\mathrm{S} 1]}\left(\mathrm{C}_{6} \mathrm{~F}_{5}\right) \mathrm{BCl}_{2}$, ${ }^{[\mathrm{S} 2]}$ and $t$-BuSiH${ }_{3}{ }^{[\mathrm{S} 3]}$ were prepared according to reported procedures. $\mathrm{MesSiH}_{3}$ was prepared according to a modified procedure. ${ }^{[\mathrm{S} 4]} \mathrm{EtMe}_{2} \mathrm{SiH}$ (purchased from Sigma Aldrich) and $\mathrm{PhSiH}_{3}$ (purchased from abcr) were degassed and stored in a glove box over $4 \AA$ molecular sieves. $\mathrm{Me}_{2} \mathrm{PhSiH}$ (purchased from abcr), $\mathrm{Ph}_{2} \mathrm{SiH}_{2}$ (purchased from abcr), and acetophenone (6, purchased from Sigma Aldrich) were distilled, degassed, and stored in a glove box over $4 \AA$ molecular sieves. Ketone 8 was purified by flash chromatography prior to use. Other ketones 9-22, tetracosane, and mesitylene were purchased from commercially sources, dried via azeotropic distillation (benzene $3 \times 3 \mathrm{~mL}$ ), degassed, and stored in a glove box. $C_{2} \mathrm{Cl}_{2}$ (purchased from Eurisotop) and $\mathrm{C}_{6} \mathrm{D}_{6}$ (purchased from Sigma Aldrich) were degassed and stored in a glove box over $4 \AA$ molecular sieves. $\mathrm{CDCl}_{3}$ (purchased from Eurisotop) was stored over $4 \AA$ molecular sieves. ${ }^{1} \mathrm{H},{ }^{11} \mathrm{~B},{ }^{13} \mathrm{C}$, ${ }^{19} \mathrm{~F},{ }^{29} \mathrm{Si}$, and ${ }^{119} \mathrm{Sn} N M R$ spectra were recorded in $\mathrm{CDCl}_{3}, \mathrm{C}_{6} \mathrm{D}_{6}$, or $\mathrm{CD}_{2} \mathrm{Cl}_{2}$ on Bruker AV 400 , Bruker AV 500 and Bruker AV 700 instruments. Chemical shifts are reported in parts per million (ppm) downfield from tetramethylsilane and are referenced to the residual solvent resonance as the internal standard $\left(\mathrm{CHCl}_{3}: \delta=7.26 \mathrm{ppm}\right.$ for ${ }^{1} \mathrm{H} \mathrm{NMR}$ and $\mathrm{CDCl}_{3}: \delta=77.16$ ppm for ${ }^{13} \mathrm{C}$ NMR, $\mathrm{C}_{6} \mathrm{D}_{5} \mathrm{H}: \delta=7.16 \mathrm{ppm}$ for ${ }^{1} \mathrm{H}$ NMR and $\mathrm{C}_{6} \mathrm{D}_{6}: \delta=128.06 \mathrm{ppm}$ for ${ }^{13} \mathrm{C}$ NMR, $\mathrm{CHDCl}_{2}: \delta=5.32 \mathrm{ppm}$ for ${ }^{1} \mathrm{H}$ NMR and $\mathrm{CD}_{2} \mathrm{Cl}_{2}: \delta=53.84 \mathrm{ppm}$ for $\left.{ }^{13} \mathrm{C} \mathrm{NMR}\right) \cdot{ }^{11} \mathrm{~B},{ }^{19} \mathrm{~F},{ }^{29} \mathrm{Si}$, and ${ }^{119} \mathrm{Sn}$ NMR spectra were calibrated according to the IUPAC recommendation using a unified chemical shift scale based on the proton resonance of trimethylsilane as primary reference. Data are reported as follows: chemical shift, multiplicity $\left(s_{b r}=\right.$ broad singlet, $s=$ singlet, $d=$ doublet, $t=$ triplet, $q=$ quartet, $m=$ multiplet, $m_{c}=$ centrosymmetric multiplet), coupling constant $(\mathrm{Hz})$, and integration. High-resolution mass spectrometry (HRMS) was performed by the Analytical Facility of the Institut für Chemie, Technische Universität Berlin. Analytical thin-layer chromatography (TLC) was performed on silica gel 60 F254 glass plates from Merck. Flash column chromatography was performed on silica gel 60 (40-63 $\mu \mathrm{m}, 230$ 400 mesh, ASTM) by Grace using the indicated solvents. Gas liquid chromatography (GLC) was performed on an Agilent Technologies 7820A gas chromatograph equipped with a FS-SE-54 capillary column $(30 \quad \mathrm{~m} \quad x \quad 0.32 \quad \mathrm{~mm}, \quad 0.25 \mu \mathrm{m}$ film thickness $)$ by CS-Chromatographie Service using the following program: $\mathrm{N}_{2}$ carrier gas, injection 
temperature $250^{\circ} \mathrm{C}$, detector temperature $300^{\circ} \mathrm{C}$, flow rate $1.7 \mathrm{~mL} / \mathrm{min}$; temperature program: start temperature $40^{\circ} \mathrm{C}$, heating rate $10^{\circ} \mathrm{C} / \mathrm{min}$, end temperature $280^{\circ} \mathrm{C}$ for $10 \mathrm{~min}$. Infrared (IR) spectra were recorded on a Agilent Technologies Cary 630 FTIR spectrophotometer equipped with an ATR unit and are reported as wave numbers $\left(\mathrm{cm}^{-1}\right)$. Melting points (M.p.) were determined with a Leica Galen III apparatus from Wagner \& Munz and are not corrected. Optical rotations were measured on a Schmidt \& Haensch Polatronic H532 polarimeter with $[\alpha]_{\lambda}$ values reported in $10^{-1}\left({ }^{\circ} \mathrm{cm}^{2} \mathrm{~g}^{-1}\right)$; concentration $c$ is in $\mathrm{g} / 100 \mathrm{~mL}$ and $\lambda$ as indicated. Enantiomeric excesses were determined by analytical high performance liquid chromatography (HPLC) analysis on an Agilent Technologies 1290 Infinity instrument with a chiral stationary phase using a Daicel Chiralcel OJ-H column, a Daicel Chiralcel OD-H column, or a Daicel Chiralcel AD-H column ( $n$-heptane/iso-propanol mixtures as solvent), or on an Agilent Technologies 1200 Infinity instrument with a stationary phase using a Daicel Chiralcel OJ-RH column (acetonitrile/water mixtures as solvent). Data for the single crystal structure determination were collected with an Agilent SuperNova diffractometer equipped with a CCD area Atlas detector and a mirror monochromator by utilizing $\mathrm{Cu}-\mathrm{K}_{\mathrm{a}}$ radiation $(\lambda=$ $1.5418 \AA$ ). Software packages used: CrysAlis PRO for data collection, cell refinement, and data reduction, ${ }^{[\mathrm{S} 5]}$ SHELXS-97 for structure solution, ${ }^{\left[{ }^{[6]}\right.}$ SHELXL-97 for structure refinement ${ }^{[S 7]}$ and Mercury 3.1.1 ${ }^{[\mathrm{S} 8]}$ for graphics. 


\section{General Procedures (GPs)}

\subsection{GP 1: General Procedure for the Optimization of the Borane-Catalyzed Hydrosilylation of Acetophenone (6)}

In a glove box, catalyst (S)-3 (2.4 mol \%), (S)-3·3,3-DMO (2.4 mol \%), or (S)-3.DMS (1.0-5.0 mol \%), acetophenone (6, 1.0 equiv), the indicated hydrosilane (1.0-5.0 equiv), and tetracosane ( $\sim 2 \mathrm{mg}$, as internal standard) are weighed into a GLC vial and 1,2- $\mathrm{F}_{2} \mathrm{C}_{6} \mathrm{H}_{4}$ (four drops) or no solvent is added. The resulting reaction mixture is stirred at room temperature for the indicated time. The progress of the reaction is monitored by GLC analysis. Direct hydrolysis and purification of the mixture by flash chromatography on silica gel ( $n$-pentane:ethyl acetate $=50: 1 \rightarrow 9: 1$ ) affords the title compound $(S)-7$ as a colorless liquid.

\subsection{GP 2: General Procedure for Borane-Catalyzed Hydrosilylation of Ketones}

In a glove box, catalyst (S)-3.DMS (2.4 mol \%), the indicated ketone (1.0 equiv), $\mathrm{PhSiH}_{3}(3.0$ or 6.0 equiv), and mesitylene ( $3 \mathrm{mg}$, as internal standard) are weighed into a GLC vial and $1,2-\mathrm{F}_{2} \mathrm{C}_{6} \mathrm{H}_{4}$ (ten drops) or no solvent is added. The resulting reaction mixture is stirred at room temperature for the indicated time. The progress of the reaction is monitored by GLC analysis. Direct hydrolysis and purification of the mixture by flash chromatography on silica gel ( $n$-pentane:ethyl acetate $=50: 1 \rightarrow 9: 1)$ affords the enantiomerically enriched alcohol.

\subsection{GP 3: General Procedure for Preparation of Racemic Alcohols}

The indicated ketone (300-600 mg, 1.0 equiv) is dissolved in iso-propanol $(5.0-10 \mathrm{~mL})$ or methanol $(5.0 \mathrm{~mL})$ and $\mathrm{NaBH}_{4}\left(0.4-0.6\right.$ equiv) is added at $0^{\circ} \mathrm{C}$. After $5 \mathrm{~min}$, the reaction mixture is warmed to room temperature and stirred overnight. The progress of the reaction is monitored by TLC analysis. After full conversion the reaction mixture is warmed to $60^{\circ} \mathrm{C}$ for $10 \mathrm{~min}$. The mixture is cooled to room temperature, and the volatiles are removed under reduced pressure. The residue is diluted with ethyl acetate $(50 \mathrm{~mL})$ and washed with $\mathrm{H}_{2} \mathrm{O}(3$ $\times 15 \mathrm{~mL})$, saturated aqueous $\mathrm{NaHCO}_{3}$ solution $(2 \times 15 \mathrm{~mL})$, and saturated aqueous $\mathrm{NaCl}$ solution $(15 \mathrm{~mL})$. The organic phase is dried over $\mathrm{MgSO}_{4}$ and concentrated under reduced pressure. Purification of the residue by flash chromatography on silica gel ( $n$-pentane:ethyl acetate $=50: 1 \rightarrow 9: 1)$ affords the racemic alcohol. 


\section{Experimental Details for the Preparation of Borane Adducts (S)-3·3,3-DMO and (S)-3.DMS}

3.1 (S)-4-(Pentafluorophenyl)-4,5-dihydro-3H-(3,3'-diphenyl)dinaphtho[2,1-c:1',2'-e] borepine [(S)-3]

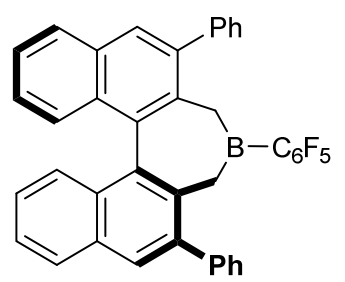

(S)-3

$$
\mathrm{C}_{40} \mathrm{H}_{24} \mathrm{BF}_{5}
$$

$\mathrm{M}=610.43 \mathrm{~g} / \mathrm{mol}$

In a glove box, a Schlenk tube was charged with dihydrostannepine (S)-4 (307 mg, 423 umol, 1.00 equiv) and $\mathrm{CH}_{2} \mathrm{Cl}_{2}(1.2 \mathrm{~mL}) .\left(\mathrm{C}_{6} \mathrm{~F}_{5}\right) \mathrm{BCl}_{2}$ (123 mg, $494 \mu \mathrm{mol}, 1.17$ equiv) was added. The Schlenk tube was closed, and the mixture was stirred at room temperature for six days. The progress of the reaction was monitored by NMR analysis. After full conversion, the reaction mixture was removed from the glove box and connected to a Schlenk line under nitrogen atmosphere. The volatiles were removed in high vacuum, and the resulting orange residue was transferred to a glove box. The residue was dissolved in $1,2-\mathrm{F}_{2} \mathrm{C}_{6} \mathrm{H}_{4}(2 \mathrm{~mL})$ and the mixture was stored at $-35^{\circ}$ overnight. The cold mixture was filtered over a PTFE syringe filter. Removal of the solvent of the filtrate in high vacuum at $50^{\circ} \mathrm{C}$ afforded dihydroborepine (S)-3 (243 mg, $319 \mu \mathrm{mol}, 94 \%)$ as an orange solid along with $\mathrm{TMSM}_{2} \mathrm{SnCl}_{2}$ (5) in a ratio of 1:0.8.

Analytical Data for dihydroborepin (S)-3: ${ }^{1} \mathrm{H}$ NMR $\left(500 \mathrm{MHz}, \mathrm{CD}_{2} \mathrm{Cl}_{2}\right): \delta=2.83$ (d, AB spin system, $J=14.6 \mathrm{~Hz}, 2 \mathrm{H}, \mathrm{BCH}{ }_{2}$ ), 3.17 (d, AB spin system, $\mathrm{J}=14.6 \mathrm{~Hz}, 2 \mathrm{H}, \mathrm{BCH}_{2}$ ), 7.20-7.30 $(\mathrm{m}, 8 \mathrm{H}), 7.31-7.41(\mathrm{~m}, 6 \mathrm{H}), 7.46-7.52(\mathrm{~m}, 2 \mathrm{H}), 7.87(\mathrm{~s}, 2 \mathrm{H}), 7.94(\mathrm{~d}, J=8.2 \mathrm{~Hz}, 2 \mathrm{H}) \mathrm{ppm}$. ${ }^{13} \mathrm{C}\left\{{ }^{1} \mathrm{H}\right\}$ NMR $\left(126 \mathrm{MHz}, \mathrm{CD}_{2} \mathrm{Cl}_{2}\right): \delta=31.0\left(\mathrm{~s}_{\mathrm{br}}, \mathrm{BCH}_{2}\right), 126.3,126.5,127.5,127.6,128.5$, $128.6,129.7,129.9,130.0,132.2,132.7,138.1,141.7,141.9 \mathrm{ppm}$. The signal for the ipso-carbon atom of $\mathrm{C}_{6} \mathrm{~F}_{5}$ was not detected. ${ }^{11} \mathrm{~B} \mathrm{NMR}\left(161 \mathrm{MHz}, \mathrm{CD}_{2} \mathrm{Cl}_{2}\right): \delta=54.2\left(\mathrm{~s}_{\mathrm{br}}\right) \mathrm{ppm}$. ${ }^{19} \mathrm{~F}\left\{{ }^{1} \mathrm{H}\right\}$ NMR $\left(471 \mathrm{MHz}, \mathrm{CD}_{2} \mathrm{Cl}_{2}\right): \delta=-163.9\left(\mathrm{~m}_{\mathrm{c}}, 2 \mathrm{~F}, m-\mathrm{C}_{6} \mathrm{~F}_{5}\right),-152.8(\mathrm{t}, \mathrm{J}=20.0 \mathrm{~Hz}, 1 \mathrm{~F}$, p- $\left.\mathrm{C}_{6} \mathrm{~F}_{5}\right),-131.9\left(\mathrm{~m}_{\mathrm{c}}, 2 \mathrm{~F}, \mathrm{o}-\mathrm{C}_{6} \mathrm{~F}_{5}\right)$ ppm. ${ }^{19} \mathrm{~F}{ }^{13} \mathrm{C}$ HMQC NMR $\left(659 / 176 \mathrm{MHz}, \mathrm{CD}_{2} \mathrm{Cl}_{2}\right): \delta=$ $-163.9 / 136.9\left(m-\mathrm{C}_{6} \mathrm{~F}_{5}\right),-152.8 / 142.0\left(p-\mathrm{C}_{6} \mathrm{~F}_{5}\right),-131.9 / 147.7\left(o-\mathrm{C}_{6} \mathrm{~F}_{5}\right)$ ppm. HRMS (APCI) calculated for $\mathrm{C}_{34} \mathrm{H}_{23}{ }^{+}\left[\left(\mathrm{M}+\mathrm{H}-\mathrm{H}_{2} \mathrm{BC}_{6} \mathrm{~F}_{5}\right)^{+}\right]$: 431.1794; found: 431.1792. 
Analytical Data for $\mathrm{TMSM}_{2} \mathrm{SnCl}_{2}$ (5): ${ }^{1} \mathrm{H}$ NMR $\left(500 \mathrm{MHz}, \mathrm{CD}_{2} \mathrm{Cl}_{2}\right.$ ): $\delta=0.19$ (s, 18H), 0.85 (s, 4H) ppm. ${ }^{13} \mathrm{C}\left\{{ }^{1} \mathrm{H}\right\}$ NMR $\left(126 \mathrm{MHz}, \mathrm{CD}_{2} \mathrm{Cl}_{2}\right): \delta=1.1,13.5 .{ }^{29} \mathrm{Si}\left\{{ }^{1} \mathrm{H}\right\}$ DEPT NMR (99 MHz, $\left.\mathrm{CD}_{2} \mathrm{Cl}_{2}\right): \delta=2.7 \mathrm{ppm} .{ }^{119} \mathrm{Sn}\left\{{ }^{1} \mathrm{H}\right\} \operatorname{NMR}\left(187 \mathrm{MHz}, \mathrm{CD}_{2} \mathrm{Cl}_{2}\right): \delta=148.7\left(\mathrm{~s}_{\mathrm{br}}\right) \mathrm{ppm}$.

The analytical data are in accordance with those reported. ${ }^{[\mathrm{S} 1]}$

\section{2 (S)-4-(Pentafluorophenyl)-4,5-dihydro-3H-(3,3'-diphenyl)dinaphtho[2,1-c:1',2'-e]} borepine 3,3-Dimethyloxetane Adduct [(S)-3·3,3-DMO]

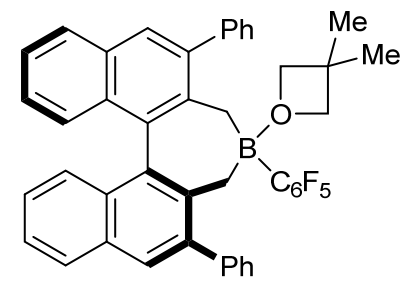

$$
\begin{gathered}
(\mathrm{S})-3 \cdot 3,3-\mathrm{DMO} \\
\mathrm{C}_{45} \mathrm{H}_{34} \mathrm{BF}_{5} \mathrm{O} \\
\mathrm{M}=696.57 \mathrm{~g} / \mathrm{mol}
\end{gathered}
$$

In a glove box, dihydroborepine (S)-3 (contaminated with 5, $21.0 \mathrm{mg}, 21.5 \mu \mathrm{mol}, 1.00$ equiv) was weighed into a GLC vial, and a solution of 3,3-dimethyloxetane (3,3-DMO) and $n$-hexane $(1: 20,0.3 \mathrm{~mL})$ was added. The suspension was shaken for $5 \mathrm{~min}$ until a white solid precipitated. The suspension was maintained overnight at room temperature. The supernatant was removed, and the precipitate was washed with $n$-hexane $(2 \times 0.2 \mathrm{~mL})$. Drying in high vacuum at $50^{\circ} \mathrm{C}$ afforded the title compound (S)-3.3,3-DMO (12.0 mg, 17.2 $\mu \mathrm{mol}, 80 \%)$ as a white solid with only traces of 5 in a ratio of $1: \leq 0.01$.

${ }^{1} \mathrm{H}$ NMR (400 MHz, $\mathrm{C}_{6} \mathrm{D}_{6}$ ): $\delta=1.07(\mathrm{~s}, 6 \mathrm{H}), 1.77$ (d, AB spin system, $\mathrm{J}=13.3 \mathrm{~Hz}, 2 \mathrm{H}$ ), 2.64 (d, AB spin system, $J=13.3 \mathrm{~Hz}, 2 \mathrm{H}$ ), 3.95 (d, A'B' spin system, $\mathrm{J}=5.6 \mathrm{~Hz}, 2 \mathrm{H}$ ), 4.02 (d, A'B' spin system, $J=5.6 \mathrm{~Hz}, 2 \mathrm{H}), 7.16-7.21(\mathrm{~m}, 4 \mathrm{H}), 7.28-7.45(\mathrm{~m}, 12 \mathrm{H}), 7.75(\mathrm{~s}, 2 \mathrm{H}), 7.88$ (d, $\mathrm{J}=8.1 \mathrm{~Hz}, 2 \mathrm{H}) \mathrm{ppm} .{ }^{13} \mathrm{C}\left\{{ }^{1} \mathrm{H}\right\}$ NMR $\left(126 \mathrm{MHz}, \mathrm{CD}_{2} \mathrm{Cl}_{2}\right): \delta=25.1,25.7,33.5,88.9,125.0$, $125.8,127.0,128.3,128.4,128.8,130.3,131.9,132.2,135.6,137.6,140.6,142.8$ ppm. The signal for the ipso-carbon atom of $\mathrm{C}_{6} \mathrm{~F}_{5}$ was not detected. ${ }^{11} \mathrm{~B}$ NMR $\left(161 \mathrm{MHz}, \mathrm{CD}_{2} \mathrm{Cl}_{2}\right)$ : $\delta=15.2\left(\mathrm{~s}_{\mathrm{br}}\right) \mathrm{ppm} .{ }^{19} \mathrm{~F}\left\{{ }^{1} \mathrm{H}\right\}$ NMR $\left(471 \mathrm{MHz}, \mathrm{CD}_{2} \mathrm{Cl}_{2}\right): \delta=-164.3\left(\mathrm{~m}_{\mathrm{c}}, 2 \mathrm{~F}, m-\mathrm{C}_{6} \mathrm{~F}_{5}\right),-157.1$ $\left(\mathrm{m}_{\mathrm{c}}, 1 \mathrm{~F}, p-\mathrm{C}_{6} F_{5}\right),-130.8\left(\mathrm{~m}_{\mathrm{c}}, 2 \mathrm{~F}, o-\mathrm{C}_{6} F_{5}\right)$ ppm. ${ }^{19} \mathrm{Fl}^{13} \mathrm{C}$ HMQC NMR $\left(659 / 176 \mathrm{MHz}, \mathrm{CD}_{2} \mathrm{Cl}_{2}\right)$ : $\delta=-164.3 / 136.9\left(m-\mathrm{C}_{6} \mathrm{~F}_{5}\right),-157.1 / 140.1\left(p-\mathrm{C}_{6} \mathrm{~F}_{5}\right),-130.8 / 148.9\left(o-\mathrm{C}_{6} \mathrm{~F}_{5}\right) \mathrm{ppm}$. 


\section{3 (S)-4-(Pentafluorophenyl)-4,5-dihydro-3H-(3,3'-diphenyl)dinaphtho[2,1-c:1',2'-e]} borepine Dimethyl Sulfide Adduct [(S)-3-DMS]

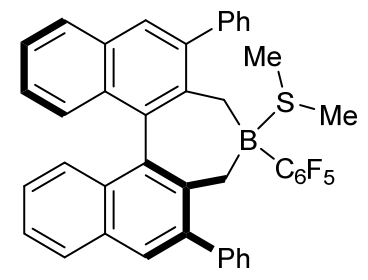

$$
\begin{gathered}
(\mathrm{S})-3 \cdot \mathrm{DMS} \\
\mathrm{C}_{42} \mathrm{H}_{30} \mathrm{BF}_{5} \mathrm{~S} \\
M=672.56 \mathrm{~g} / \mathrm{mol}
\end{gathered}
$$

In a glove box, dihydroborepine (S)-3 (contaminated with 5, $57.0 \mathrm{mg}, 58.5 \mu \mathrm{mol}, 1.00$ equiv) was weighed into a GLC vial, and a solution of dimethyl sulfide (DMS) and $n$-hexane (1:20, $0.3 \mathrm{~mL}$ ) was added. The suspension was shaken for $5 \mathrm{~min}$ and stirred with a spatula until a white solid precipitated. The supernatant was removed, and the precipitate was washed with a solution of DMS and $n$-hexane $(6 \times 0.2 \mathrm{~mL})$. Drying in high vacuum afforded the title compound (S)-3.DMS (20.8 mg, $30.9 \mu \mathrm{mol}, 53 \%)$ as a white solid with only traces of $\mathbf{5}$ in a ratio of $1: \leq 0.01$. Singe crystals of (S)-3.DMS suitable for X-ray diffraction were obtained by slow vaporization of a $n$-hexane solution.

${ }^{1} \mathrm{H}$ NMR $\left(700 \mathrm{MHz}, \mathrm{CD}_{2} \mathrm{Cl}_{2}\right): \delta=1.62(\mathrm{~s}, 6 \mathrm{H}), 2.15(\mathrm{~d}, \mathrm{AB}$ spin system, $\mathrm{J}=13.8 \mathrm{~Hz}, 2 \mathrm{H}), 2.98$ (d, AB spin system, $J=13.8 \mathrm{~Hz}, 2 \mathrm{H}), 7.21-7.24(\mathrm{~m}, 4 \mathrm{H}), 7.28-7.39(\mathrm{~m}, 10 \mathrm{H}), 7.40-7.44(\mathrm{~m}$, $2 \mathrm{H}), 7.77(\mathrm{~s}, 2 \mathrm{H}), 7.90(\mathrm{~d}, \mathrm{~J}=8.1 \mathrm{~Hz}, 2 \mathrm{H}) \mathrm{ppm} .{ }^{13} \mathrm{C}\left\{{ }^{1} \mathrm{H}\right\}$ NMR $\left(126 \mathrm{MHz}, \mathrm{CD}_{2} \mathrm{Cl}_{2}\right): \delta=18.5$, 26.1, 125.3, 125.9, 127.1, 127.2, 128.4, 128.4, 129.0, 130.5, 132.1, 135.9, 136.4, 141.0, $142.7 \mathrm{ppm}$. The signal for the ipso-carbon atom of $\mathrm{C}_{6} \mathrm{~F}_{5}$ was not detected. ${ }^{11} \mathrm{~B}$ NMR (161 $\left.\mathrm{MHz}, \mathrm{CD}_{2} \mathrm{Cl}_{2}\right): \delta=-21.5\left(\mathrm{~s}_{\mathrm{br}}\right) \mathrm{ppm} .{ }^{19} \mathrm{~F}\left\{{ }^{1} \mathrm{H}\right\}$ NMR $\left(471 \mathrm{MHz}, \mathrm{CD}_{2} \mathrm{Cl}_{2}\right): \delta=-163.7\left(\mathrm{~m}_{\mathrm{c}}, 2 \mathrm{~F}\right.$, $\left.m-\mathrm{C}_{6} F_{5}\right),-156.0\left(\mathrm{~m}_{\mathrm{c}}, 1 \mathrm{~F}, p-\mathrm{C}_{6} F_{5}\right),-129.2\left(\mathrm{~m}_{\mathrm{c}}, 2 \mathrm{~F}, \mathrm{o}_{-} \mathrm{C}_{6} \mathrm{~F}_{5}\right)$ ppm. ${ }^{19} \mathrm{~F} /{ }^{13} \mathrm{C}$ HMQC NMR $\left(659 / 176 \mathrm{MHz}, \mathrm{CD}_{2} \mathrm{Cl}_{2}\right): \delta=-163.7 / 136.8\left(m-\mathrm{C}_{6} \mathrm{~F}_{5}\right),-156.0 / 140.3\left(p-\mathrm{C}_{6} \mathrm{~F}_{5}\right),-129.2 / 148.9$ $\left(0-\mathrm{C}_{6} \mathrm{~F}_{5}\right)$ ppm. Molecular structure and X-ray data: CCDC-1469970 contains the supplementary crystallographic data for this compound. These data can be obtained free of charge at www.ccdc.cam.ac.uk/conts/retrieving.html [or from the Cambridge Crystallographic Data Centre, 12 Union Road, Cambridge CB2 1EZ, UK; fax: (internat.) +44(1223)336-033, Email: deposit@ccdc.cam.ac.uk]. 


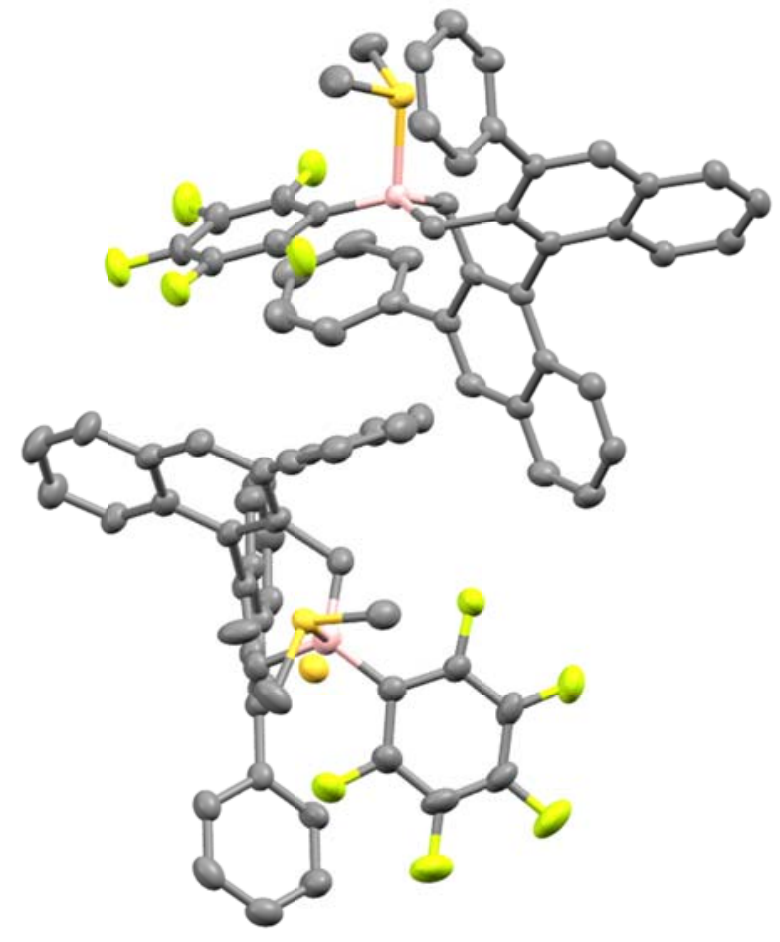

Figure S1. Molecular structure of (S)-3.DMS. Thermal ellipsoids represent 50\% probability level. $\mathrm{H}$ atoms are omitted for clarity.

Empirical formula

Formula weight

Temperature

Wavelength

Crystal system

Space group

Unit cell dimensions

Volume

Z

Density (calculated)

Absorption coefficient

$\mathrm{F}(000)$

Crystal size

Theta range for data collection

Index ranges
$\mathrm{C}_{42} \mathrm{H}_{30} \mathrm{BF}_{5} \mathrm{~S}$ [(S)-3.DMS]

$672.53 \mathrm{~g} / \mathrm{mol}$

$150.00(10) \mathrm{K}$

\section{$1.54184 \AA$}

Monoclinic

P21

$$
\begin{array}{ll}
a=11.1346(2) \AA & \alpha=90^{\circ} . \\
b=10.5539(2) \AA & \beta=98.7877(17)^{\circ} . \\
c=28.3860(6) \AA & \gamma=90^{\circ} .
\end{array}
$$$$
\text { 3296.56(11) } \AA^{3}
$$

4

$1.355 \mathrm{Mg} / \mathrm{m}^{3}$

$1.371 \mathrm{~mm}^{-1}$

1392

$0.13 \times 0.11 \times 0.11 \mathrm{~mm}^{3}$

3.15 to $67.49^{\circ}$.

$-13<=\mathrm{h}<=13,-12<=\mathrm{k}<=10,-34<=\mathrm{l}<=33$ 
Reflections collected

Independent reflections

Completeness to theta $=67.49^{\circ}$

Absorption correction

Max. and min. transmission

Refinement method

Data / restraints / parameters

Goodness-of-fit on $\mathrm{F}^{2}$

Final $\mathrm{R}$ indices [l>2sigma(l)]

$\mathrm{R}$ indices (all data)

Absolute structure parameter

Largest diff. peak and hole
12650

$8306[R($ int $)=0.0333]$

$100.0 \%$

Semi-empirical from equivalents

0.8638 and 0.8419

Full-matrix least-squares on $\mathrm{F}^{2}$

8306 / 1 / 904

1.065

$\mathrm{R} 1=0.0425, \mathrm{wR} 2=0.1003$

$\mathrm{R} 1=0.0513, \mathrm{wR} 2=0.1124$

$0.035(18)$

0.251 and -0.265 e. $\AA^{-3}$ 


\section{Optimization of the Borane-Catalyzed Hydrosilylation of Acetophenone}

Table S1. Screening of Hydrosilanes. ${ }^{a}$<smiles>CC(=O)c1ccccc1</smiles>

6
(S)-3·DMS (2.4 mol \%)

hydrosilane ( 1.0 equiv)

$1,2-\mathrm{F}_{2} \mathrm{C}_{6} \mathrm{H}_{4}$

r.t.

$2 d$

$(S)-7$ (after hydrolysis)<smiles>CN(C)B1Cc2c(-c3ccccc3)cc3ccccc3c2-c2c(-c3ccccc3)cc3ccccc3c2C1</smiles>

(S)-3·DMS

\begin{tabular}{cccc} 
entry & hydrosilane & conv. $(\%)^{b}$ & ee $(\%)^{c}$ \\
\hline 1 & $\mathrm{Ph}_{3} \mathrm{SiH}$ & - & - \\
2 & $\mathrm{Me}_{2} \mathrm{PhSiH}$ & - & - \\
3 & $\mathrm{EtMe}_{2} \mathrm{SiH}$ & - & - \\
4 & $\mathrm{MePhSiH}_{2}$ & quant. & 81 \\
5 & $\mathrm{PhSiH}_{3}$ & quant. & 87 \\
6 & $\mathrm{MesSiH}_{3}$ & quant. & -
\end{tabular}

${ }^{a}$ All reactions were performed according to GP $1 .{ }^{b}$ Determined by GLC analysis using tetracosane as internal standard. ${ }^{c}$ Determined by HPLC analysis using a chiral stationary phase.

Table S2. Screening of the Amount of $\mathrm{PhSiH}_{3}{ }^{a}$<smiles>N#CC(=O)c1ccccc1</smiles>

6
(S)-3.DMS (2.4 mol \%)<smiles>C[C@H](O)c1ccccc1</smiles>

(S)-7 (after hydrolysis)<smiles></smiles>

(S)-3.DMS

\begin{tabular}{ccccc} 
entry & $\mathrm{PhSiH}_{3}$ (equiv) & solvent & conv. $(\%)^{b}$ & ee $(\%)^{c}$ \\
\hline 1 & 2.0 & $1,2-\mathrm{F}_{2} \mathrm{C}_{6} \mathrm{H}_{4}$ & quant. & 93 \\
2 & 3.0 & $1,2-\mathrm{F}_{2} \mathrm{C}_{6} \mathrm{H}_{4}$ & quant. & 93 \\
3 & 3.0 & neat & quant. & 93 \\
4 & 5.0 & neat & quant. & 93
\end{tabular}

${ }^{a}$ All reactions were performed according to GP 1. ${ }^{b}$ Determined by GLC analysis using tetracosane as internal standard. ${ }^{c}$ Determined by HPLC analysis using a chiral stationary phase. 
Table S3. Screening of Catalyst Loading. ${ }^{a}$<smiles>CC(=O)c1ccccc1</smiles>

6
(S)-3·DMS<smiles>CC(O)c1ccccc1</smiles>

(S)-7 (after hydrolysis)<smiles>CN(C)B1Cc2c(-c3ccccc3)cc3ccccc3c2-c2c(-c3ccccc3)cc3ccccc3c2C1</smiles>

(S)-3.DMS

\begin{tabular}{cccc} 
entry & $(\mathrm{S})-3 \cdot \mathrm{DMS}(\mathrm{mol} \%)$ & conv. $(\%)^{b}$ & ee $(\%)^{c}$ \\
\hline 1 & 1.0 & 42 & 80 \\
2 & 2.4 & quant. & 93 \\
3 & 5.0 & quant. & 94
\end{tabular}

${ }^{a}$ All reactions were performed according to GP $1 .{ }^{b}$ Determined by GLC analysis using tetracosane as internal standard. ${ }^{c}$ Determined by HPLC analysis using a chiral stationary phase.

Table S4. Hydrosilylation with Different Catalysts. ${ }^{a}$<smiles>CC(=O)c1ccccc1</smiles>

6<smiles>CC(O)c1ccccc1</smiles>

(S)-7 (after hydrolysis)

\begin{tabular}{cccccc} 
entry & borane & time & conv. $(\%)^{b}$ & yield $(\%)$ & ee $(\%)^{c}$ \\
\hline 1 & $(S)-3^{d}$ & $1 \mathrm{~d}$ & quant. & 86 & 92 \\
2 & $(S)-3 \cdot 3,3-\mathrm{DMO}$ & $2 \mathrm{~d}$ & quant. & 56 & 90 \\
3 & $(S)-3 \cdot \mathrm{DMS}$ & $1 \mathrm{~d}$ & quant. & 62 & 93
\end{tabular}

${ }^{a}$ All reactions were performed according to GP $1 .{ }^{b}$ Determined by GLC analysis using tetracosane as internal standard. ${ }^{c}$ Determined by HPLC analysis using a chiral stationary phase. ${ }^{d}(S)-3$ along with $\mathrm{TMSM}_{2} \mathrm{SnCl}_{2}(\mathbf{5})$ in a ratio of 1:0.8. 


\section{$5 \quad$ Procedures and Characterization Data for Alcohols 7 and 23-37}

(S)-1-Phenylethanol [(S)-7]

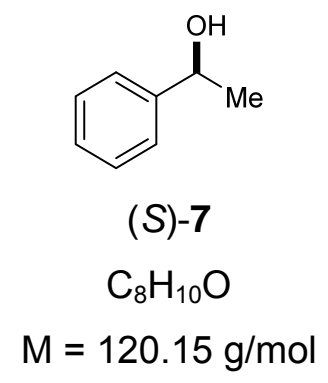

Prepared according to GP 2 from (S)-3.DMS (3.0 mg, $4.0 \mu \mathrm{mol}, 2.4 \mathrm{~mol} \%$ ), acetophenone (6, $22.0 \mathrm{mg}, 0.183 \mathrm{mmol}, 1.00$ equiv), and $\mathrm{PhSiH}_{3}$ (59.0 mg, $549 \mu \mathrm{mol}, 3.00$ equiv). Purification after one day afforded the title compound (S)-7 (13.9 mg, $114 \mu \mathrm{mol}, 62 \%, 93 \%$ ee) as a colorless liquid.

${ }^{1} \mathrm{H}$ NMR (400 MHz, $\left.\mathrm{C}_{6} \mathrm{D}_{6}\right): \delta=1.13\left(\mathrm{~s}_{\mathrm{br}}, 1 \mathrm{H}\right), 1.27(\mathrm{~d}, \mathrm{~J}=6.5 \mathrm{~Hz}, 3 \mathrm{H}), 4.51(\mathrm{q}, \mathrm{J}=6.5,1 \mathrm{H})$, 7.08 (tt, $J=7.3 \mathrm{~Hz}, J=1.5 \mathrm{~Hz}, 1 \mathrm{H}), 7.14-7.18(\mathrm{~m}, 2 \mathrm{H}), 7.19-7.23(\mathrm{~m}, 2 \mathrm{H}) \mathrm{ppm} .{ }^{13} \mathrm{C}\left\{{ }^{1} \mathrm{H}\right\}$ NMR $\left(101 \mathrm{MHz}, \mathrm{C}_{6} \mathrm{D}_{6}\right): \delta=25.6,70.2,125.6,127.4,128.6,146.8$ ppm. HRMS (APCI) calculated for $\mathrm{C}_{8} \mathrm{H}_{11} \mathrm{O}^{+}\left[\mathrm{M}^{+}\right]$: 123.0804; found: 123.0791. IR (ATR): $\tilde{v}=3269,2012,1602$, 1493, 1450, 1408, 1368, 1203, 1075, 1029, 1009, 897, 758, $697 \mathrm{~cm}^{-1}$. Optical rotation: $[\alpha]_{D}^{20}=-48^{\circ}\left(\mathrm{c}=0.17, \mathrm{CHCl}_{3}, 93 \%\right.$ ee $)$. The enantiomeric excess was determined by HPLC analysis on a chiral stationary phase (Daicel Chiralcel OJ-H column, column temperature $20^{\circ} \mathrm{C}$, solvent $n$-heptane:iso-propanol $=98: 2$, flow rate $0.8 \mathrm{~mL} / \mathrm{min}, \lambda=210 \mathrm{~nm}$ ): $t_{\mathrm{R}}=25.8$ $\min$ for $(S)-7, t_{\mathrm{R}}=30.8 \mathrm{~min}$ for $(R)-7$.

The analytical data are in accordance with those reported. ${ }^{[\mathrm{S} 9]}$

(S)-1-(4'-Trifluoromethylphenyl)ethanol [(S)-23]<smiles>CC(O)c1ccc(C(F)(F)F)cc1</smiles>

(S)-23

$\mathrm{C}_{9} \mathrm{H}_{9} \mathrm{~F}_{3} \mathrm{O}$

$\mathrm{M}=190.17 \mathrm{~g} / \mathrm{mol}$ 
Prepared according to GP 2 from (S)-3.DMS (2.6 mg, $3.9 \mu \mathrm{mol}, 2.4 \mathrm{~mol} \%$ ), 4-trifluoromethylacetophenone (9, $22.0 \mathrm{mg}, 117 \mu \mathrm{mol}, 1.00$ equiv), and $\mathrm{PhSiH}_{3}(38.0 \mathrm{mg}, 351$ $\mu \mathrm{mol}, 3.00$ equiv). Purification after four days afforded the title compound (S)-23 (12.8 mg, $67.3 \mu \mathrm{mol}, 57 \%, 82 \%$ ee) as a colorless liquid.

${ }^{1} \mathrm{H}$ NMR $\left(500 \mathrm{MHz}, \mathrm{CDCl}_{3}\right): \delta=1.51(\mathrm{~d}, \mathrm{~J}=6.5 \mathrm{~Hz}, 3 \mathrm{H}), 1.91(\mathrm{~d}, \mathrm{~J}=3.6 \mathrm{~Hz}, 1 \mathrm{H}), 4.97\left(\mathrm{~m}_{\mathrm{c}}\right.$, $1 \mathrm{H}), 7.49\left(\mathrm{~m}_{\mathrm{c}}, 2 \mathrm{H}\right), 7.61\left(\mathrm{~m}_{\mathrm{c}}, 2 \mathrm{H}\right) \mathrm{ppm} .{ }^{13} \mathrm{C}\left\{{ }^{1} \mathrm{H}\right\} \mathrm{NMR}\left(126 \mathrm{MHz}, \mathrm{CDCl}_{3}\right): \delta=25.6,70.0$, 123.2, 125.6, 125.7, 129.8, 149.8 ppm. $\left.{ }^{13} \mathrm{~F}^{1} \mathrm{H}\right\}$ NMR (471 MHz, $\left.\mathrm{CDCl}_{3}\right): \delta=-62.5 \mathrm{ppm}$. HRMS (El) calculated for $\mathrm{C}_{9} \mathrm{H}_{9} \mathrm{~F}_{3} \mathrm{O}^{+}\left[\mathrm{M}^{+}\right]$: 190.0600; found: 190.0605. IR (ATR): $\tilde{v}=3305$, 2161, 1621, 1450, 1414, 1372, 1322, 1206, 1162, 1114, 1088, 1065, 1015, 898, 838, 737 $\mathrm{cm}^{-1}$. Optical rotation: $[\alpha]_{D}^{20}=-32^{\circ}\left(\mathrm{c}=0.39, \mathrm{CHCl}_{3}, 82 \%\right.$ ee $)$. The enantiomeric excess was determined by HPLC analysis on a chiral stationary phase (Daicel Chiralcel AD-H column, column temperature $20^{\circ} \mathrm{C}$, solvent $n$-heptane:iso-propanol $=99.7: 0.3$, flow rate 0.8 $\mathrm{mL} / \mathrm{min}, \lambda=210 \mathrm{~nm}): t_{\mathrm{R}}=75.1 \mathrm{~min}$ for $(S)-23, t_{\mathrm{R}}=79.3 \mathrm{~min}$ for $(R)-23$.

The analytical data are in accordance with those reported. ${ }^{[\mathrm{S} 9]}$

\section{(S)-1-(4'-Nitrophenyl)ethanol [(S)-24]}

$$
\begin{aligned}
& (S)-24 \\
& C_{8} \mathrm{H}_{9} \mathrm{NO}_{3} \\
& M=167.16 \mathrm{~g} / \mathrm{mol}
\end{aligned}
$$

Prepared according to GP 2 from (S)-3.DMS (2.1 mg, $3.2 \mu \mathrm{mol}, 2.4 \mathrm{~mol} \%$ ), 4-nitroacetophenone (9, $22.0 \mathrm{mg}, 133 \mu \mathrm{mol}, 1.00$ equiv), and $\mathrm{PhSiH}_{3}(86.0 \mathrm{mg}, 799 \mu \mathrm{mol}$, 6.00 equiv). Purification after two days afforded the title compound (S)-24 (12.5 mg, 74.8 $\mu \mathrm{mol}, 56 \%, 80 \%$ ee) as a yellow solution.

${ }^{1} \mathrm{H}$ NMR $\left(400 \mathrm{MHz}, \mathrm{CDCl}_{3}\right): \delta=1.52(\mathrm{~d}, \mathrm{~J}=6.6 \mathrm{~Hz}, 3 \mathrm{H}), 1.93(\mathrm{~d}, \mathrm{~J}=3.8 \mathrm{~Hz}, 1 \mathrm{H}), 4.99-5.07$ $(\mathrm{m}, 1 \mathrm{H}), 7.55\left(\mathrm{~m}_{\mathrm{c}}, 2 \mathrm{H}\right), 8.21\left(\mathrm{~m}_{\mathrm{c}}, 2 \mathrm{H}\right) \mathrm{ppm} .{ }^{13} \mathrm{C}\left\{{ }^{1} \mathrm{H}\right\} \mathrm{NMR}\left(101 \mathrm{MHz}, \mathrm{CDCl}_{3}\right): \delta=25.7,69.7$, 123.9, 126.3, 147.4, 153.1 ppm. HRMS (El) calculated for $\mathrm{C}_{7} \mathrm{H}_{6} \mathrm{NO}_{3}{ }^{+}\left[\left(\mathrm{M}-\mathrm{CH}_{3}\right)^{+}\right]$: 152.0342 ; found: 152.0340. IR (ATR): $\tilde{v}=2981,2215,1602,1511,1452,1412,1341,1288,1200$, $1108,1087,1011,899,852,769,751,698 \mathrm{~cm}^{-1}$. Optical rotation: $[\alpha]_{D}^{20}=-17^{\circ}(\mathrm{c}=0.65$, $\mathrm{CHCl}_{3}, 80 \%$ ee). The enantiomeric excess was determined by HPLC analysis on a chiral 
stationary phase (Daicel Chiralcel $\mathrm{OJ}-\mathrm{H}$ column, column temperature $20^{\circ} \mathrm{C}$, solvent $n$-heptane:iso-propanol $=95: 5$, flow rate $0.8 \mathrm{~mL} / \mathrm{min}, \lambda=210 \mathrm{~nm}): t_{\mathrm{R}}=40.6 \mathrm{~min}$ for $(S)-24, t_{\mathrm{R}}$ $=44.8 \mathrm{~min}$ for $(R)-24$.

The analytical data are in accordance with those reported. ${ }^{[\mathrm{S} 9]}$

\section{(S)-Methyl-4-(1-hydroxyethyl)benzoate [(S)-25]}<smiles>CC(=O)c1ccc(C(C)O)cc1</smiles>

(S)-25

$\mathrm{C}_{10} \mathrm{H}_{12} \mathrm{O}_{3}$

$\mathrm{M}=180.20 \mathrm{~g} / \mathrm{mol}$

Prepared according to GP 2 from (S)-3.DMS (1.6 mg, $2.4 \mu \mathrm{mol}, 2.4 \mathrm{~mol} \%$ ), methyl 4-acetylbenzoate (10, 18.0 mg, $101 \mu \mathrm{mol}, 1.00$ equiv), $\mathrm{PhSiH}_{3}(33.0 \mathrm{mg}, 303 \mu \mathrm{mol}, 3.00$ equiv), and 1,2- $\mathrm{F}_{2} \mathrm{C}_{6} \mathrm{H}_{4}$ (10 drops). Purification after one days afforded the title compound (S)-25 (15.9 mg, $88.2 \mu \mathrm{mol}, 87 \%, 98 \%$ ee) as a colorless liquid.

${ }^{1} \mathrm{H}$ NMR $\left(400 \mathrm{MHz}, \mathrm{CDCl}_{3}\right): \delta=1.50(\mathrm{~d}, \mathrm{~J}=6.6 \mathrm{~Hz}, 3 \mathrm{H}), 1.90(\mathrm{~d}, \mathrm{~J}=3.8 \mathrm{~Hz}, 1 \mathrm{H}), 3.91(\mathrm{~s}$, $3 \mathrm{H}), 4.92-5.01(\mathrm{~m}, 1 \mathrm{H}), 7.44\left(\mathrm{~m}_{\mathrm{c}}, 2 \mathrm{H}\right), 8.02\left(\mathrm{~m}_{\mathrm{c}}, 2 \mathrm{H}\right) \mathrm{ppm} .{ }^{13} \mathrm{C}\left\{{ }^{1} \mathrm{H}\right\}$ NMR $\left(101 \mathrm{MHz}, \mathrm{CDCl}_{3}\right): \delta$ $=25.5,52.2,70.1,125.4,129.4,130.0,151.0,167.1$ ppm. HRMS (EI) calculated for $\mathrm{C}_{10} \mathrm{H}_{12} \mathrm{O}_{3}{ }^{+}\left[\mathrm{M}^{+}\right]:$180.0781; found: 180.0778. IR (ATR): $\tilde{v}=2988,2158,2007,1718,1701$, $1611,1576,1435,1414,1368,1274,1192,1109,1087,1017,964,898,857,830,773,706$ $\mathrm{cm}^{-1}$. Optical rotation: $[\alpha]_{D}^{20}=-38^{\circ}\left(\mathrm{c}=1.36, \mathrm{CHCl}_{3}, 98 \%\right.$ ee $)$. The enantiomeric excess was determined by HPLC analysis on a chiral stationary phase (Daicel Chiralcel OJ-H column, column temperature $20^{\circ} \mathrm{C}$, solvent $n$-heptane:iso-propanol $=97: 3$, flow rate 0.8 $\mathrm{mL} / \mathrm{min}, \lambda=210 \mathrm{~nm}): t_{\mathrm{R}}=50.3 \mathrm{~min}$ for $(S)-25, t_{\mathrm{R}}=54.8 \mathrm{~min}$ for $(R)-25$.

The analytical data are in accordance with those reported. ${ }^{[\mathrm{S} 10]}$ 


\section{(S)-1-(4'-Chlorophenyl)ethanol [(S)-26]}<smiles>CC(O)c1ccc(Cl)cc1</smiles>

(S)-26

$\mathrm{C}_{8} \mathrm{H}_{9} \mathrm{ClO}$

$M=156.61 \mathrm{~g} / \mathrm{mol}$

Prepared according to GP 2 from (S)-3.DMS (2.3 mg, $3.4 \mu \mathrm{mol}, 2.4 \mathrm{~mol} \%$ ), 4-chloroacetophenone (11, 22 mg, $142 \mu \mathrm{mol}, 1.00$ equiv), and $\mathrm{PhSiH}_{3}(46.0 \mathrm{mg}, 427 \mu \mathrm{mol}$, 3.00 equiv). Purification after one day afforded the title compound (S)-26 (14.2 mg, 90.7 $\mu \mathrm{mol}, 63 \%, 95 \%$ ee) as a colorless liquid.

${ }^{1} \mathrm{H}$ NMR $\left(400 \mathrm{MHz}, \mathrm{C}_{6} \mathrm{D}_{6}\right): \delta=0.95(\mathrm{~d}, \mathrm{~J}=3.8 \mathrm{~Hz}, 1 \mathrm{H}), 1.12(\mathrm{~d}, \mathrm{~J}=6.2 \mathrm{~Hz}, 3 \mathrm{H}), 4.30$ (dq, $J=6.2 \mathrm{~Hz}, J=3.8 \mathrm{~Hz}, 1 \mathrm{H}), 6.89\left(\mathrm{~m}_{\mathrm{c}}, 2 \mathrm{H}\right), 7.11\left(\mathrm{~m}_{\mathrm{c}}, 2 \mathrm{H}\right) \mathrm{ppm} .{ }^{13} \mathrm{C}\left\{{ }^{1} \mathrm{H}\right\} \operatorname{NMR}\left(101 \mathrm{MHz}, \mathrm{C}_{6} \mathrm{D}_{6}\right)$ : $\delta=25.5,69.4,127.0,128.6,133.0,145.2 \mathrm{ppm}$. HRMS (EI) calculated for $\mathrm{C}_{8} \mathrm{H}_{9} \mathrm{ClO}^{+}\left[\mathrm{M}^{+}\right]$: 156.0336; found: 156.0336. IR (ATR): $\tilde{v}=2979$, 1904, 1655, 1597, 1492, 1450, 1406, 1370, 1338, 1294, 1200, 1085, 1012, 896, 825, 777, $718 \mathrm{~cm}^{-1}$. Optical rotation: $[\alpha]_{D}^{20}=-38^{\circ}$ (c $=0.28, \mathrm{CHCl}_{3}, 95 \%$ ee). The enantiomeric excess was determined by HPLC analysis on a chiral stationary phase (Daicel Chiralcel OJ-H column, column temperature $20^{\circ} \mathrm{C}$, solvent $n$-heptane:iso-propanol $=98: 2$, flow rate $0.8 \mathrm{~mL} / \mathrm{min}, \lambda=210 \mathrm{~nm}): t_{\mathrm{R}}=25.3 \mathrm{~min}$ for $(S)-\mathbf{2 6}$, $t_{\mathrm{R}}=28.1 \mathrm{~min}$ for $(R)-26$.

The analytical data are in accordance with those reported. ${ }^{[\mathrm{S} 9]}$

\section{(S)-1-(4'-Methylphenyl)ethanol [(S)-27]}

$$
\begin{aligned}
& (S)-27 \\
& M=136.19 \mathrm{~g} / \mathrm{mol}
\end{aligned}
$$

Prepared according to GP 2 from (S)-3.DMS (2.6 mg, $3.9 \mu \mathrm{mol}, 2.4 \mathrm{~mol} \%$ ), 4-methylacetophenone (12, $22.0 \mathrm{mg}, 164 \mu \mathrm{mol}, 1.00$ equiv), and $\mathrm{PhSiH}_{3}(53.0 \mathrm{mg}, 492 \mu \mathrm{mol}$, 
3.00 equiv). Purification after two days afforded the title compound (S)-27 (15.1 mg, 111 $\mu \mathrm{mol}, 67 \%, 85 \%$ ee) as a colorless liquid.

${ }^{1} \mathrm{H}$ NMR $\left(400 \mathrm{MHz}, \mathrm{CDCl}_{3}\right): \delta=1.48(\mathrm{~d}, \mathrm{~J}=6.5 \mathrm{~Hz}, 3 \mathrm{H}), 1.74(\mathrm{bs}, 1 \mathrm{H}), 2.35(\mathrm{~s}, 3 \mathrm{H}), 4.88$ (q, $J=6.5 \mathrm{~Hz}, 1 \mathrm{H}), 7.16(\mathrm{~d}, \mathrm{~J}=7.8 \mathrm{~Hz}, 2 \mathrm{H}), 7.27(\mathrm{~d}, \mathrm{~J}=7.8 \mathrm{~Hz}, 2 \mathrm{H}) \mathrm{ppm} .{ }^{13} \mathrm{C}\left\{{ }^{1} \mathrm{H}\right\}$ NMR $(101$ $\mathrm{MHz}_{\mathrm{CDCl}}$ ): $\delta=21.2,25.2,70.4,125.5,129.3,137.3,143.0$ ppm. HRMS (EI) calculated for $\mathrm{C}_{9} \mathrm{H}_{12} \mathrm{O}^{+}\left[\mathrm{M}^{+}\right]:$136.0883; found: 136.0888. IR (ATR): $\tilde{v}=3274,2985,2008,1905,1615$, $1513,1449,1413,1367,1342,1303,1201,1180,1070,1008,941,897,815,726 \mathrm{~cm}^{-1}$. Optical rotation: $[\alpha]_{D}^{20}=-49^{\circ}\left(\mathrm{c}=0.33, \mathrm{CHCl}_{3}, 85 \%\right.$ ee) . The enantiomeric excess was determined by HPLC analysis on a chiral stationary phase (Daicel Chiralcel OJ-H column, column temperature $20^{\circ} \mathrm{C}$, solvent $n$-heptane:iso-propanol $=98: 2$, flow rate $0.8 \mathrm{~mL} / \mathrm{min}, \lambda=$ $210 \mathrm{~nm}): t_{\mathrm{R}}=27.4 \mathrm{~min}$ for $(S)-27, t_{\mathrm{R}}=31.9 \min$ for $(R)-27$.

The analytical data are in accordance with those reported. ${ }^{[\mathrm{S} 9]}$

\section{(S)-1-(4-Biphenylyl)ethanol [(S)-28]}

$$
\begin{aligned}
& (S)-28 \\
& C_{14} \mathrm{H}_{14} \mathrm{O} \\
& M=198.27 \mathrm{~g} / \mathrm{mol}
\end{aligned}
$$

Prepared according to GP 2 from (S)-3.DMS (1.7 mg, $2.6 \mu \mathrm{mol}, 2.4 \mathrm{~mol} \%$ ), 4'-phenylacetophenone (13, 21.0 mg, $107 \mu \mathrm{mol}, 1.00$ equiv), $\mathrm{PhSiH}_{3}(35.0 \mathrm{mg}, 321 \mu \mathrm{mol}$, 3.00 equiv), and $1,2-\mathrm{F}_{2} \mathrm{C}_{6} \mathrm{H}_{4}$ (10 drops). Purification after two days afforded the title compound (S)-28 (13.6 mg, $68.6 \mu \mathrm{mol}, 64 \%, 70 \%$ ee) as a white solid.

M.p.: $97-98^{\circ} \mathrm{C}$ (ethyl acetate). ${ }^{1} \mathrm{H}$ NMR $\left(400 \mathrm{MHz}, \mathrm{CDCl}_{3}\right): \delta=1.55(\mathrm{~d}, \mathrm{~J}=6.6 \mathrm{~Hz}, 3 \mathrm{H}), 1.80$ $(\mathrm{d}, J=3.6 \mathrm{~Hz}, 1 \mathrm{H}), 4.93-5.00(\mathrm{~m}, 1 \mathrm{H}), 7.32-7.37(\mathrm{~m}, 1 \mathrm{H}), 7.41-7.48(\mathrm{~m}, 4 \mathrm{H}), 7.56-7.61(\mathrm{~m}$, $4 \mathrm{H}) \mathrm{ppm} .{ }^{13} \mathrm{C}\left\{{ }^{1} \mathrm{H}\right\}$ NMR $\left(101 \mathrm{MHz}, \mathrm{CDCl}_{3}\right): \delta=25.3,70.3,126.0,127.2,127.4,128.9,140.6$, 141.0, 144.9 ppm. HRMS (El) calculated for $\mathrm{C}_{14} \mathrm{H}_{14} \mathrm{O}^{+}\left[\mathrm{M}^{+}\right]:$198.1039; found: 198.1045. IR (ATR): $\tilde{v}=3033,2974,2930,1563,1482,1450,1400,1343,1288,1243,1192,1121,1067$, $1021,1004,944,893,834,759,726,685 \mathrm{~cm}^{-1}$. Optical rotation: $[\alpha]_{D}^{20}=-30^{\circ}(\mathrm{c}=0.94$, $\mathrm{CHCl}_{3}, 70 \%$ ee). The enantiomeric excess was determined by HPLC analysis on a chiral stationary phase (Daicel Chiralcel $\mathrm{OD}-\mathrm{RH}$ column, column temperature $20^{\circ} \mathrm{C}$, solvent 
acetonitrile:water $=40: 60$, flow rate $0.4 \mathrm{~mL} / \mathrm{min}, \lambda=210 \mathrm{~nm}): t_{\mathrm{R}}=38.7 \mathrm{~min}$ for $(S)-28, t_{\mathrm{R}}=$ $42.8 \mathrm{~min}$ for $(R)-28$.

The analytical data are in accordance with those reported. ${ }^{[11]}$

\section{(S)-1-(4'-Methoxyphenyl)ethanol [(S)-29]}

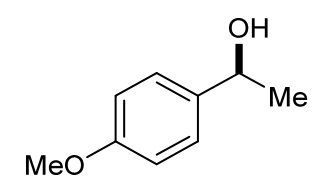

(S)-29

$$
\mathrm{C}_{9} \mathrm{H}_{12} \mathrm{O}_{2}
$$

$\mathrm{M}=152.19 \mathrm{~g} / \mathrm{mol}$

Prepared according to GP 2 from (S)-3.DMS (2.6 mg, $3.9 \mu \mathrm{mol}, 2.4 \mathrm{~mol} \%$ ), 4-methoxyacetophenone (14, $22.0 \mathrm{mg}, 146 \mu \mathrm{mol}, 1.00$ equiv), and $\mathrm{PhSiH}_{3}$ (48.0 mg, 439 $\mu \mathrm{mol}, 3.00$ equiv). Purification after one day afforded the title compound (S)-29 (14.3 mg, $94.0 \mu \mathrm{mol}, 64 \%, 28 \%$ ee) as a colorless liquid.

${ }^{1} \mathbf{H}$ NMR $\left(400 \mathrm{MHz}, \mathrm{CDCl}_{3}\right): \delta=1.48(\mathrm{~d}, \mathrm{~J}=6.5 \mathrm{~Hz}, 3 \mathrm{H}), 1.67(\mathrm{~d}, \mathrm{~J}=3.5 \mathrm{~Hz}, 1 \mathrm{H}), 3.81(\mathrm{~s}$, $3 \mathrm{H}), 4.86\left(\mathrm{~m}_{\mathrm{c}}, 1 \mathrm{H}\right), 6.88\left(\mathrm{~m}_{\mathrm{c}}, 2 \mathrm{H}\right), 7.30\left(\mathrm{~m}_{\mathrm{c}}, 2 \mathrm{H}\right) \mathrm{ppm} .{ }^{13} \mathrm{C}\left\{{ }^{1} \mathrm{H}\right\}$ NMR $\left(101 \mathrm{MHz}, \mathrm{CDCl}_{3}\right): \delta=$ 25.2, 55.4, 70.1, 114.0, 126.8, 138.1, 159.1 ppm. HRMS (EI) calculated for $\mathrm{C}_{9} \mathrm{H}_{11} \mathrm{O}^{+}[\mathrm{M}-\mathrm{H}]^{+}$: 151.0758; found: 151.0754. IR (ATR): $\tilde{v}=3387,2978,2161,1611,1586,1510,1458,1420$, $1368,1325,1301,1240,1204,1173,1116,1086,1067,1033,1004,895,829 \mathrm{~cm}^{-1}$. Optical rotation: $[\alpha]_{D}^{20}=-13^{\circ}\left(\mathrm{c}=0.18, \mathrm{CHCl}_{3}, 28 \%\right.$ ee $)$. The enantiomeric excess was determined by HPLC analysis on a chiral stationary phase (Daicel Chiralcel OD-H column, column temperature $20^{\circ} \mathrm{C}$, solvent $n$-heptane:iso-propanol $=98: 2$, flow rate $0.8 \mathrm{~mL} / \mathrm{min}, \lambda=210 \mathrm{~nm}$ ): $t_{\mathrm{R}}=31.1 \mathrm{~min}$ for $(R)-29, t_{\mathrm{R}}=34.7 \mathrm{~min}$ for $(S)-29$.

The analytical data are in accordance with those reported. ${ }^{[\mathrm{S} 12]}$ 


\section{(S)-1-(2',4',6'-Trimethylphenyl)ethanol [(S)-30]}

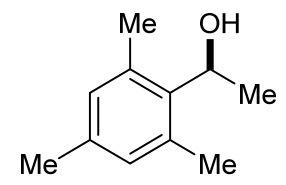

(S)-30

$\mathrm{C}_{11} \mathrm{H}_{16} \mathrm{O}$

$M=164.25 \mathrm{~g} / \mathrm{mol}$

Prepared according to GP 2 from (S)-3.DMS (2.6 mg, $3.9 \mu \mathrm{mol}, 2.4 \mathrm{~mol} \%$ ), 2,4,6-trimethylacetophenone (15, $22.0 \mathrm{mg}, 136 \mu \mathrm{mol}, 1.00$ equiv), and $\mathrm{PhSiH}_{3}$ (44.0 mg, 407 $\mu \mathrm{mol}, 3.00$ equiv). Purification after four days afforded the title compound (S)-30 (2.8 mg, $17.0 \mu \mathrm{mol}, 12 \%, 17 \%$ ee) as a colorless solid.

M.p.: $65-67^{\circ} \mathrm{C}$ (ethyl acetate). ${ }^{1} \mathrm{H}$ NMR $\left(400 \mathrm{MHz}, \mathrm{CDCl}_{3}\right): \delta=1.52(\mathrm{~d}, \mathrm{~J}=6.7 \mathrm{~Hz}, 3 \mathrm{H}), 1.66$ $\left(\mathrm{s}_{\mathrm{br}}, 1 \mathrm{H}\right), 2.25(\mathrm{~s}, 3 \mathrm{H}), 2.42(\mathrm{~s}, 6 \mathrm{H}), 5.37(\mathrm{q}, \mathrm{J}=6.7 \mathrm{~Hz}, 1 \mathrm{H}), 6.82(\mathrm{~s}, 2 \mathrm{H}) \mathrm{ppm} .{ }^{13} \mathrm{C}\left\{{ }^{1} \mathrm{H}\right\} \mathrm{NMR}$ $\left(101 \mathrm{MHz}, \mathrm{CDCl}_{3}\right): \delta=20.7,20.8,21.7,67.7,130.3,135.8,136.6,137.7$ ppm. HRMS (EI) calculated for $\mathrm{C}_{11} \mathrm{H}_{16} \mathrm{O}[\mathrm{M}]^{+}:$164.1196; found: 164.1193. IR (ATR): $\tilde{v}=2975,2923,2872$, $2731,2185,1725,1610,1576,1445,1363,1311,1245,1210,1150,1072,1030,999,958$, 925, 891, 849, 743, 719, $662 \mathrm{~cm}^{-1}$. Optical rotation: $[\alpha]_{D}^{20}=-12^{\circ}\left(\mathrm{c}=0.29, \mathrm{CHCl}_{3}, 17 \%\right.$ ee $)$. The enantiomeric excess was determined by HPLC analysis on a chiral stationary phase (Daicel Chiralcel OD-H column, column temperature $20^{\circ} \mathrm{C}$, solvent $n$-heptane:iso-propanol = 99:1, flow rate $0.8 \mathrm{~mL} / \mathrm{min}, \lambda=210 \mathrm{~nm}): t_{\mathrm{R}}=22.4 \mathrm{~min}$ for $(S)-30, t_{\mathrm{R}}=25.0 \mathrm{~min}$ for $(R)-30$.

The analytical data are in accordance with those reported. ${ }^{[\mathrm{S} 10]}$

\section{(S)-1-(4'-Bromophenyl)ethanol [(S)-31]}<smiles>CC(O)c1ccc(Br)cc1</smiles>

(S)-31

$\mathrm{C}_{8} \mathrm{H}_{9} \mathrm{BrO}$

$M=201.06 \mathrm{~g} / \mathrm{mol}$

Prepared according to GP 2 from (S)-3.DMS (1.8 mg, $2.7 \mu \mathrm{mol}, 2.4 \mathrm{~mol} \%$ ), 4-bromoacetophenone (16, $22.0 \mathrm{mg}, 111 \mu \mathrm{mol}, 1.00$ equiv), and $\mathrm{PhSiH}_{3}(36.0 \mathrm{mg}, 332 \mu \mathrm{mol}$, 
3.00 equiv). Purification after one day afforded the title compound (S)-31 (14.0 mg, 69.6 $\mu \mathrm{mol}, 63 \%, 95 \%$ ee) as a colorless liquid.

${ }^{1} \mathrm{H}$ NMR (400 MHz, $\left.\mathrm{C}_{6} \mathrm{D}_{6}\right): \delta=0.99-1.07\left(\mathrm{~s}_{\mathrm{br}}, 1 \mathrm{H}\right), 1.21(\mathrm{~d}, \mathrm{~J}=6.3 \mathrm{~Hz}, 3 \mathrm{H}), 4.37$ (q, J = 6.3 $\mathrm{Hz}, 1 \mathrm{H}), 6.92\left(\mathrm{~m}_{\mathrm{c}}, 2 \mathrm{H}\right), 7.36\left(\mathrm{~m}_{\mathrm{c}}, 2 \mathrm{H}\right) \mathrm{ppm} .{ }^{13} \mathrm{C}\left\{{ }^{1} \mathrm{H}\right\}$ NMR $\left(101 \mathrm{MHz}, \mathrm{C}_{6} \mathrm{D}_{6}\right): \delta=25.5,69.4$, 121.1, 127.4, 131.6, 145.7 ppm. HRMS (El) calculated for $\mathrm{C}_{8} \mathrm{H}_{9} \mathrm{BrO}^{+}\left[\mathrm{M}^{+}\right]$: 199.9829; found: 199.9831. IR (ATR): $\tilde{v}=3300,2975,1903,1593,1488,1448,1401,1369,1335,1294,1200$, $1111,1068,1008,896,820,770,715 \mathrm{~cm}^{-1}$. Optical rotation: $[\alpha]_{D}^{20}=-32^{\circ}\left(\mathrm{c}=0.36, \mathrm{CHCl}_{3}\right.$, $95 \%$ ee). The enantiomeric excess was determined by HPLC analysis on a chiral stationary phase (Daicel Chiralcel OJ-H column, column temperature $20^{\circ} \mathrm{C}$, solvent $n$-heptane:iso-propanol $=98: 2$, flow rate $0.8 \mathrm{~mL} / \mathrm{min}, \lambda=210 \mathrm{~nm}$ ): $t_{\mathrm{R}}=28.7 \mathrm{~min}$ for $(S)-\mathbf{3 1}$, $t_{\mathrm{R}}=32.0 \mathrm{~min}$ for $(R)-31$.

The analytical data are in accordance with those reported. ${ }^{[\mathrm{S} 9]}$

\section{(S)-1-(3'-Bromophenyl)ethanol [(S)-32]}<smiles>CC(O)c1cccc(Br)c1</smiles>

(S)-32

$\mathrm{C}_{8} \mathrm{H}_{9} \mathrm{BrO}$

$M=201.06 \mathrm{~g} / \mathrm{mol}$

Prepared according to GP 2 from (S)-3.DMS (1.8 mg, $2.7 \mu \mathrm{mol}, 2.4 \mathrm{~mol} \%$ ), 3-bromoacetophenone (17, $22.0 \mathrm{mg}, 111 \mu \mathrm{mol}, 1.00$ equiv), and $\mathrm{PhSiH}_{3}(36.0 \mathrm{mg}, 332 \mu \mathrm{mol}$, 3.00 equiv). Purification after one day afforded the title compound (S)-32 (17.5 mg, 87.0 $\mu \mathrm{mol}, 78 \%, 99 \%$ ee) as a colorless liquid.

${ }^{1} \mathrm{H}$ NMR $\left(400 \mathrm{MHz}, \mathrm{C}_{6} \mathrm{D}_{6}\right): \delta=0.97-1.04\left(\mathrm{~s}_{\mathrm{br}}, 1 \mathrm{H}\right), 1.18(\mathrm{~d}, \mathrm{~J}=6.5 \mathrm{~Hz}, 3 \mathrm{H}), 4.37$ (q, J = 6.5 $\mathrm{Hz}, 1 \mathrm{H}), 6.85(\mathrm{t}, J=7.7 \mathrm{~Hz}, 1 \mathrm{H}), 7.02\left(\mathrm{~m}_{\mathrm{c}}, 1 \mathrm{H}\right), 7.27-7.31(\mathrm{~m}, 1 \mathrm{H}), 7.50-7.53(\mathrm{~m}, 1 \mathrm{H}) \mathrm{ppm}$. ${ }^{13} \mathrm{C}\left\{{ }^{1} \mathrm{H}\right\}$ NMR $\left(101 \mathrm{MHz}, \mathrm{C}_{6} \mathrm{D}_{6}\right): \delta=25.4,69.4,122.8,124.1,128.9,130.1,130.4,149.2 \mathrm{ppm}$. HRMS (EI) calculated for $\mathrm{C}_{8} \mathrm{H}_{9} \mathrm{BrO}^{+}\left[\mathrm{M}^{+}\right]$: 199.9841; found: 199.9831. IR (ATR): $\tilde{v}=3340$, 2985, 1595, 1568, 1475, 1449, 1426, 1369, 1334, 1296, 1257, 1198, 1107, 1066, 1009, 903, $780,694,663 \mathrm{~cm}^{-1}$. Optical rotation: $[\alpha]_{D}^{20}=-35^{\circ}$ (c $=0.32, \mathrm{CHCl}_{3}, 99 \%$ ee). The enantiomeric excess was determined by HPLC analysis on a chiral stationary phase (Daicel 
Chiralcel OJ-H column, column temperature $20^{\circ} \mathrm{C}$, solvent $n$-heptane:iso-propanol $=98: 2$, flow rate $0.8 \mathrm{~mL} / \mathrm{min}, \lambda=210 \mathrm{~nm}): t_{\mathrm{R}}=25.1 \mathrm{~min}$ for $(S)-32, t_{\mathrm{R}}=30.2 \mathrm{~min}$ for $(R)-32$.

The analytical data are in accordance with those reported. ${ }^{[\mathrm{S} 9]}$

(S)-1-(2'-Bromophenyl)ethanol [(S)-33]<smiles>CC(O)c1ccccc1Br</smiles>

(S)-33

$\mathrm{C}_{8} \mathrm{H}_{9} \mathrm{BrO}$

$M=201.06 \mathrm{~g} / \mathrm{mol}$

Prepared according to GP 2 from (S)-3.DMS (1.8 mg, $2.7 \mu \mathrm{mol}, 2.4 \mathrm{~mol} \%$ ), 2-bromoacetophenone (18, $22 \mathrm{mg}, 0.11 \mathrm{mmol}, 1.0$ equiv), and $\mathrm{PhSiH}_{3}$ (36 mg, $0.49 \mathrm{mmol}$, 3.0 equiv). Purification after four days afforded the title compound (S)-33 (10.0 mg, 49.7 $\mu \mathrm{mol}, 45 \%, 73 \%$ ee) as a colorless liquid.

${ }^{1} \mathrm{H}$ NMR $\left(500 \mathrm{MHz}, \mathrm{CDCl}_{3}\right): \delta=1.39(\mathrm{~d}, \mathrm{~J}=6.5 \mathrm{~Hz}, 3 \mathrm{H}), 1.91(\mathrm{~d}, \mathrm{~J}=3.3 \mathrm{~Hz}, 1 \mathrm{H}), 5.14$ (dq, $J=6.5 \mathrm{~Hz}, J=3.3 \mathrm{~Hz}, 1 \mathrm{H}), 7.03\left(\mathrm{~m}_{\mathrm{c}}, 1 \mathrm{H}\right), 7.24\left(\mathrm{~m}_{\mathrm{c}}, 1 \mathrm{H}\right), 7.41\left(\mathrm{~m}_{\mathrm{c}}, 1 \mathrm{H}\right), 7.49\left(\mathrm{~m}_{\mathrm{c}}, 1 \mathrm{H}\right) \mathrm{ppm}$. ${ }^{13} \mathrm{C}\left\{{ }^{1} \mathrm{H}\right\}$ NMR $\left(126 \mathrm{MHz}, \mathrm{CDCl}_{3}\right): \delta=23.7,69.4,121.9,126.8,128.0,128.9,132.8,144.7$ ppm. HRMS (EI) calculated for $\mathrm{C}_{8} \mathrm{H}_{9} \mathrm{BrO}^{+}\left[\mathrm{M}^{+}\right]$: 199.9836; found: 199.9831. IR (ATR): $\tilde{v}=3392,2933,1591,1568,1468,1439,1367,1343,1261,1199,1127,1090,1069,1045$, $1023,1006,945,897,750,722,667 \mathrm{~cm}^{-1}$. Optical rotation: $[\alpha]_{D}^{20}=-44^{\circ}\left(\mathrm{c}=0.29, \mathrm{CHCl}_{3}\right.$, $73 \%$ ee). The enantiomeric excess was determined by HPLC analysis on a chiral stationary phase (Daicel Chiralcel OJ-H column, column temperature $20^{\circ} \mathrm{C}$, solvent $n$-heptane:iso-propanol $=98: 2$, flow rate $0.8 \mathrm{~mL} / \mathrm{min}, \lambda=210 \mathrm{~nm}$ ): $t_{\mathrm{R}}=20.0 \mathrm{~min}$ for $(S)-33$, $t_{\mathrm{R}}=21.7 \mathrm{~min}$ for $(R)-33$.

The analytical data are in accordance with those reported. ${ }^{[\mathrm{S} 9]}$ 


\section{(S)-1,2-Diphenylethanol [(S)-34]}<smiles>OC(Cc1ccccc1)c1ccccc1</smiles>

(S)-34

$\mathrm{C}_{14} \mathrm{H}_{14} \mathrm{O}$

$M=198.27 \mathrm{~g} / \mathrm{mol}$

Prepared according to GP 2 from (S)-3.DMS (1.8 mg, $2.7 \mu \mathrm{mol}, 2.4 \mathrm{~mol} \%$ ), 2-phenylacetophenone (19, $22.0 \mathrm{mg}, 112 \mu \mathrm{mol}, 1.00$ equiv), and $\mathrm{PhSiH}_{3}(36.0 \mathrm{mg}, 336 \mu \mathrm{mol}$, 3.00 equiv). Purification after four days afforded the title compound (S)-34 (9.0 mg, 45.4 $\mu \mathrm{mol}, 40 \%, 74 \%$ ee) as a white solid.

M.p.: $66-67^{\circ} \mathrm{C}$ (ethyl acetate). ${ }^{1} \mathbf{H}$ NMR $\left(400 \mathrm{MHz}, \mathrm{CDCl}_{3}\right): \delta=1.93(\mathrm{~d}, \mathrm{~J}=3 \mathrm{~Hz}, 1 \mathrm{H}), 3.00$ (dd, $J=13.3 \mathrm{~Hz}, J=8.6 \mathrm{~Hz}, 1 \mathrm{H}), 3.06(\mathrm{dd}, J=13.3 \mathrm{~Hz}, J=8.6 \mathrm{~Hz}, 1 \mathrm{H}), 4.90\left(\mathrm{~m}_{\mathrm{c}}, 1 \mathrm{H}\right), 7.19$ $7.22(\mathrm{~m}, 2 \mathrm{H}), 7.23-7.26(\mathrm{~m}, 1 \mathrm{H}), 7.28-7.33(\mathrm{~m}, 3 \mathrm{H}), 7.33-7.38(\mathrm{~m}, 4 \mathrm{H}) \mathrm{ppm} .{ }^{13} \mathrm{C}\left\{{ }^{1} \mathrm{H}\right\}$ NMR $\left(175 \mathrm{MHz}, \mathrm{CDCl}_{3}\right): \delta=46.3,75.5,126.0,126.8,127.8,128.6,128.7,129.7,138.2,144.0$ ppm. HRMS (APCl) calculated for $\mathrm{C}_{14} \mathrm{H}_{14} \mathrm{O}^{+}\left[\mathrm{M}^{+}\right]$: 198.1039; found: 198.1040. IR (ATR): $\tilde{v}=$ 2887, 1602, 1495, 1454, 1407, 1316, 1273, 1208, 1150, 1072, 1026, 952, 917, 830, 778, 760, 741, $695 \mathrm{~cm}^{-1}$. Optical rotation: $[\alpha]_{D}^{20}=-9^{\circ}\left(\mathrm{c}=0.77, \mathrm{CHCl}_{3}, 74 \%\right.$ ee). The enantiomeric excess was determined by HPLC analysis on a chiral stationary phase (Daicel Chiralcel OD-RH column, column temperature $20^{\circ} \mathrm{C}$, solvent acetonitrile:water $=40: 60$, flow rate $0.4 \mathrm{~mL} / \mathrm{min}, \lambda=210 \mathrm{~nm}): t_{\mathrm{R}}=6.5 \mathrm{~min}$ for $(R)-34, t_{\mathrm{R}}=11.2 \mathrm{~min}$ for $(S)-34$.

The analytical data are in accordance with those reported. ${ }^{[313]}$

\section{(S)-Cyclohexyl(phenyl)methanol [(S)-35]}

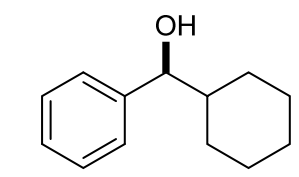

(S)-35

$$
\begin{gathered}
\mathrm{C}_{13} \mathrm{H}_{18} \mathrm{O} \\
M=190.29 \mathrm{~g} / \mathrm{mol}
\end{gathered}
$$

Prepared according to GP 2 from (S)-3.DMS (2.6 mg, $3.9 \mu \mathrm{mol}, 2.4 \mathrm{~mol} \%$ ), cyclohexyl phenyl ketone (20, $22.0 \mathrm{mg}, 117 \mu \mathrm{mol}, 1.00$ equiv), and $\mathrm{PhSiH}_{3}(38.0 \mathrm{mg}, 351 \mu \mathrm{mol}, 3.00$ 
equiv). Purification after four days afforded the title compound (S)-35 (5.3 mg, $28 \mu \mathrm{mol}, 24 \%$, $62 \%$ ee) as a colorless liquid.

${ }^{1} \mathrm{H}$ NMR $\left(400 \mathrm{MHz}, \mathrm{CDCl}_{3}\right): \delta=0.81-1.30(\mathrm{~m}, 5 \mathrm{H}), 1.37\left(\mathrm{~m}_{\mathrm{c}}, 1 \mathrm{H}\right), 1.57-1.71(\mathrm{~m}, 3 \mathrm{H}), 1.73-$ $1.78(\mathrm{~m}, 1 \mathrm{H}), 1.80\left(\mathrm{~m}_{\mathrm{c}}, 1 \mathrm{H}\right), 1.99\left(\mathrm{~m}_{\mathrm{c}}, 1 \mathrm{H}\right), 4.37\left(\mathrm{~m}_{\mathrm{c}}, 1 \mathrm{H}\right), 7.26-7.37(\mathrm{~m}, 5 \mathrm{H}) \mathrm{ppm} .{ }^{13} \mathrm{C}\left\{{ }^{1} \mathrm{H}\right\}$ NMR $\left(101 \mathrm{MHz}, \mathrm{CDCl}_{3}\right): \delta=26.1,26.2,26.6,29.0,29.5,45.1,79.5,126.7,127.6,128.3$, 143.8 ppm. HRMS (EI) calculated for $\mathrm{C}_{13} \mathrm{H}_{18} \mathrm{O}^{+}\left[\mathrm{M}^{+}\right]$: 190.1352; found: 190.1356. IR (ATR): $\tilde{v}$ = 2936, 2854, 1725, 1603, 1492, 1449, 1306, 1256, 1197, 1067, 1013, 985, 912, 892, 848, $820,758,699 \mathrm{~cm}^{-1}$. Optical rotation: $[\alpha]_{D}^{20}=-14^{\circ}\left(\mathrm{c}=0.59, \mathrm{CHCl}_{3}, 62 \%\right.$ ee $)$. The enantiomeric excess was determined by HPLC analysis on a chiral stationary phase (Daicel Chiralcel AD-H column, column temperature $20^{\circ} \mathrm{C}$, solvent $n$-heptane:iso-propanol $=99: 1$, flow rate $0.8 \mathrm{~mL} / \mathrm{min}, \lambda=210 \mathrm{~nm}): t_{\mathrm{R}}=30.4 \mathrm{~min}$ for $(S)-35, t_{\mathrm{R}}=33.7 \mathrm{~min}$ for $(R)-35$.

The analytical data are in accordance with those reported. ${ }^{\left[{ }^{[14]}\right.}$

\section{(S)-1-(2'-Naphthyl)ethanol [(S)-36]}

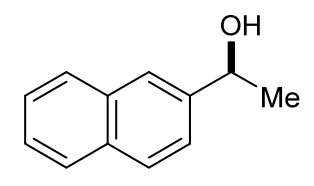

(S)-36

$\mathrm{C}_{12} \mathrm{H}_{12} \mathrm{O}$

$\mathrm{M}=172.23 \mathrm{~g} / \mathrm{mol}$

Prepared according to GP 2 from (S)-3.DMS (2.6 mg, $3.9 \mu \mathrm{mol}, 2.4 \mathrm{~mol} \%$ ), 2-acetonaphthone (21, $25.0 \mathrm{mg}, 147 \mu \mathrm{mol}, 1.00$ equiv), and $\mathrm{PhSiH}_{3}(48.0 \mathrm{mg}, 441 \mu \mathrm{mol}$, 3.00 equiv). Purification after one day afforded the title compound (S)-36 (19.5 mg, 113 $\mu \mathrm{mol}, 77 \%, 80 \%$ ee) as a white solid.

M.p.: $73-74^{\circ} \mathrm{C}$ (ethyl acetate). ${ }^{1} \mathrm{H}$ NMR $\left(400 \mathrm{MHz}, \mathrm{CDCl}_{3}\right): \delta=1.59(\mathrm{~d}, \mathrm{~J}=6.5 \mathrm{~Hz}, 3 \mathrm{H}), 1.87$ $(\mathrm{d}, J=3.5 \mathrm{~Hz}, 1 \mathrm{H}), 5.08\left(\mathrm{~m}_{\mathrm{c}}, 1 \mathrm{H}\right), 7.43-7.54(\mathrm{~m}, 3 \mathrm{H}), 7.80-7.87(\mathrm{~m}, 4 \mathrm{H}) \mathrm{ppm} .{ }^{13} \mathrm{C}\left\{{ }^{1} \mathrm{H}\right\}$ NMR $\left(101 \mathrm{MHz}, \mathrm{CDCl}_{3}\right): \delta=25.3,70.7,123.9,125.9,126.3,127.8,128.1,128.4,133.0,133.4$, 143.3 ppm. HRMS (El) calculated for $\mathrm{C}_{12} \mathrm{H}_{12} \mathrm{O}^{+}\left[\mathrm{M}^{+}\right]$: 172.0883; found: 172.0886. IR (ATR): $\tilde{v}=3270,3052,3021,2969,2921,2878,1598,1502,1450,1407,1361,1321,1273,1164$, 1123, 1071, 1023, 949, 900, 860, 823, 771, 741, $702 \mathrm{~cm}^{-1}$. Optical rotation: $[\alpha]_{D}^{20}=-47^{\circ}$ ( $\mathrm{c}=0.28, \mathrm{CHCl}_{3}, 80 \%$ ee). The enantiomeric excess was determined by HPLC analysis on a chiral stationary phase (Daicel Chiralcel $\mathrm{OJ}-\mathrm{H}$ column, column temperature $20^{\circ} \mathrm{C}$, solvent 
$n$-heptane:iso-propanol $=95: 5$, flow rate $0.8 \mathrm{~mL} / \mathrm{min}, \lambda=210 \mathrm{~nm}): t_{R}=35.9 \mathrm{~min}$ for $(S)-36$, $t_{\mathrm{R}}=47.9 \mathrm{~min}$ for $(R)-36$.

The analytical data are in accordance with those reported. ${ }^{[\mathrm{S} 9]}$

(S)-4'-Bromophenyl(phenyl)methanol [(S)-37]<smiles>OC(c1ccccc1)c1ccc(Br)cc1</smiles>

(S)-37

$\mathrm{C}_{13} \mathrm{H}_{11} \mathrm{BrO}$

$M=263.13 \mathrm{~g} / \mathrm{mol}$

Prepared according to GP 2 from (S)-3.DMS (1.4 mg, $2.0 \mu \mathrm{mol}, 2.4 \mathrm{~mol} \%$ ), 4-bromobenzophenone (22, $22.0 \mathrm{mg}, 84.0 \mu \mathrm{mol}, 1.00$ equiv), and $\mathrm{PhSiH}_{3}$ (55.0 mg, 506 $\mu \mathrm{mol}, 6.00$ equiv). Purification after four days afforded the title compound (S)-37 (14.2 mg, $54.0 \mu \mathrm{mol}, 64 \%, 14 \%$ ee) as a white solid.

M.p.: $50-52^{\circ} \mathrm{C}$ (ethyl acetate). ${ }^{1} \mathrm{H}$ NMR $\left(400 \mathrm{MHz}, \mathrm{CDCl}_{3}\right): \delta=2.20(\mathrm{~d}, \mathrm{~J}=3.5 \mathrm{~Hz}, 1 \mathrm{H}), 5.81$ $(\mathrm{d}, \mathrm{J}=3.5 \mathrm{~Hz}, 1 \mathrm{H}), 7.25-7.31(\mathrm{~m}, 3 \mathrm{H}), 7.33-7.36(\mathrm{~m}, 4 \mathrm{H}), 7.44-7.48(\mathrm{~m}, 2 \mathrm{H}) \mathrm{ppm} .{ }^{13} \mathrm{C}\left\{{ }^{1} \mathrm{H}\right\}$ NMR $\left(101 \mathrm{MHz}, \mathrm{CDCl}_{3}\right): \delta=75.9,121.6,126.7,128.1,128.4,128.8,131.7,142.9,143.5$ ppm. HRMS (APCI) calculated for $\mathrm{C}_{13} \mathrm{H}_{10} \mathrm{Br}^{+}\left[(\mathrm{M}-\mathrm{OH})^{+}\right]$: 244.9960; found: 244.9970. IR (ATR): $\tilde{v}=2138,2008,1904,1591,1483,1451,1398,1237,1182,1105,1070,1034,1008$, 917, 843, 789, 749, $697 \mathrm{~cm}^{-1}$. Optical rotation: $[\alpha]_{D}^{20}=+4^{\circ}\left(\mathrm{c}=0.27, \mathrm{CHCl}_{3}, 14 \%\right.$ ee). The enantiomeric excess was determined by HPLC analysis on chiral stationary phase (Daicel Chiralcel AD-H column, column temperature $20^{\circ} \mathrm{C}$, solvent $n$-heptane:iso-propanol $=95: 5$, flow rate $0.8 \mathrm{~mL} / \mathrm{min}, \lambda=210 \mathrm{~nm}): t_{\mathrm{R}}=18.9 \mathrm{~min}$ for $(R)-37, t_{\mathrm{R}}=21.6 \mathrm{~min}$ for $(S)-37$.

The analytical data are in accordance with those reported. ${ }^{[S 15]}$ 
$6 \quad$ NMR Spectra of All Compounds 
(S)-4-(Pentafluorophenyl)-4,5-dihydro-3H-(3,3'-diphenyl)dinaphtho[2,1-c:1',2'-e] borepine [(S)-3] ${ }^{1} \mathrm{H}$ NMR $\left(500 \mathrm{MHz}, \mathrm{CD}_{2} \mathrm{Cl}_{2}\right):\left({ }^{*}=\mathrm{TMSM}_{2} \mathrm{SnCl}_{2}(\mathbf{5})\right)$
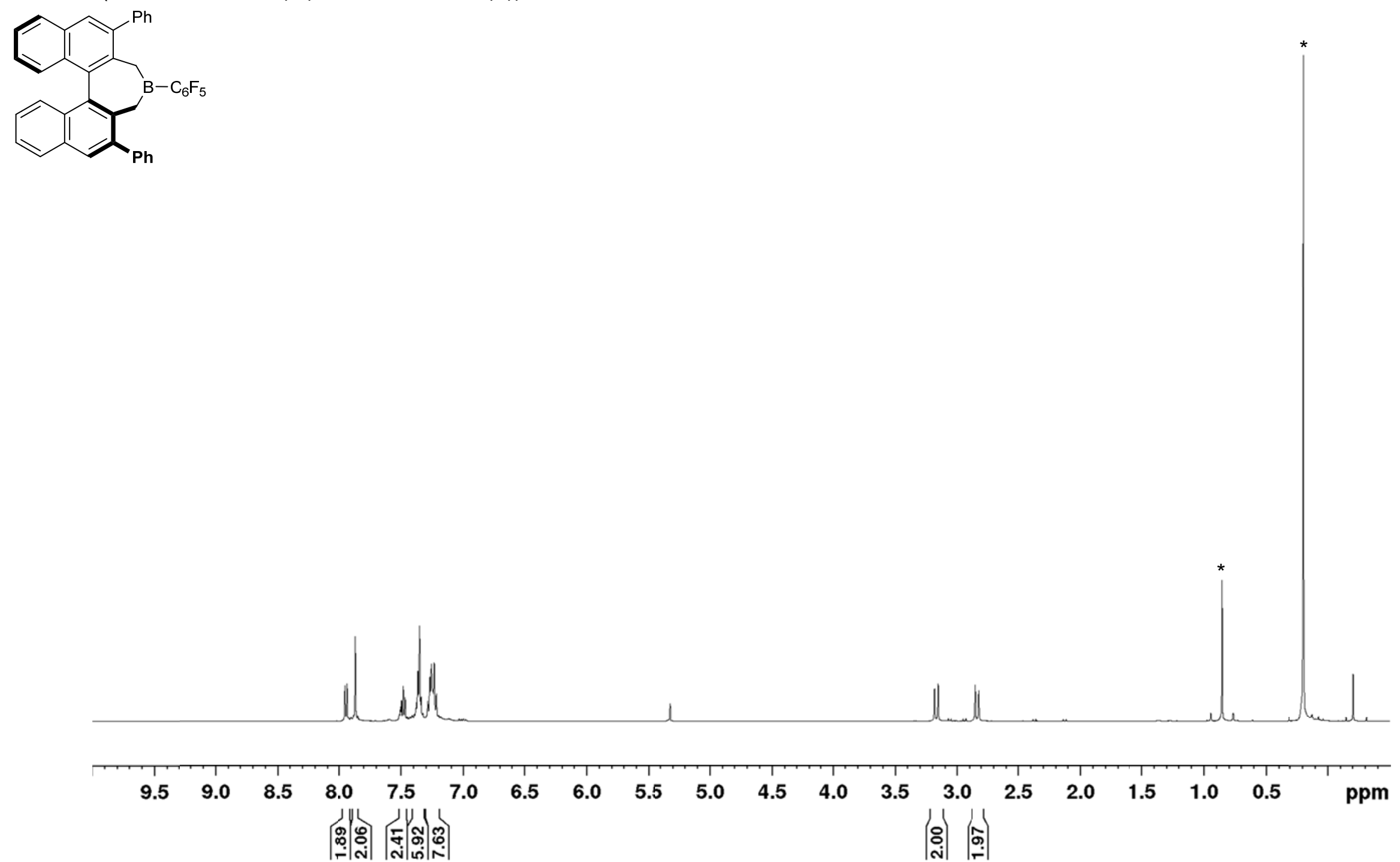
${ }^{13} \mathrm{C}\left\{{ }^{1} \mathrm{H}\right\}$ NMR $\left(126 \mathrm{MHz}, \mathrm{CD}_{2} \mathrm{Cl}_{2}\right):\left({ }^{*}=\mathrm{TMSM}_{2} \mathrm{SnCl}_{2}(5)\right)$

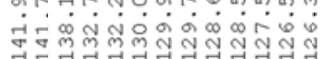

WNill

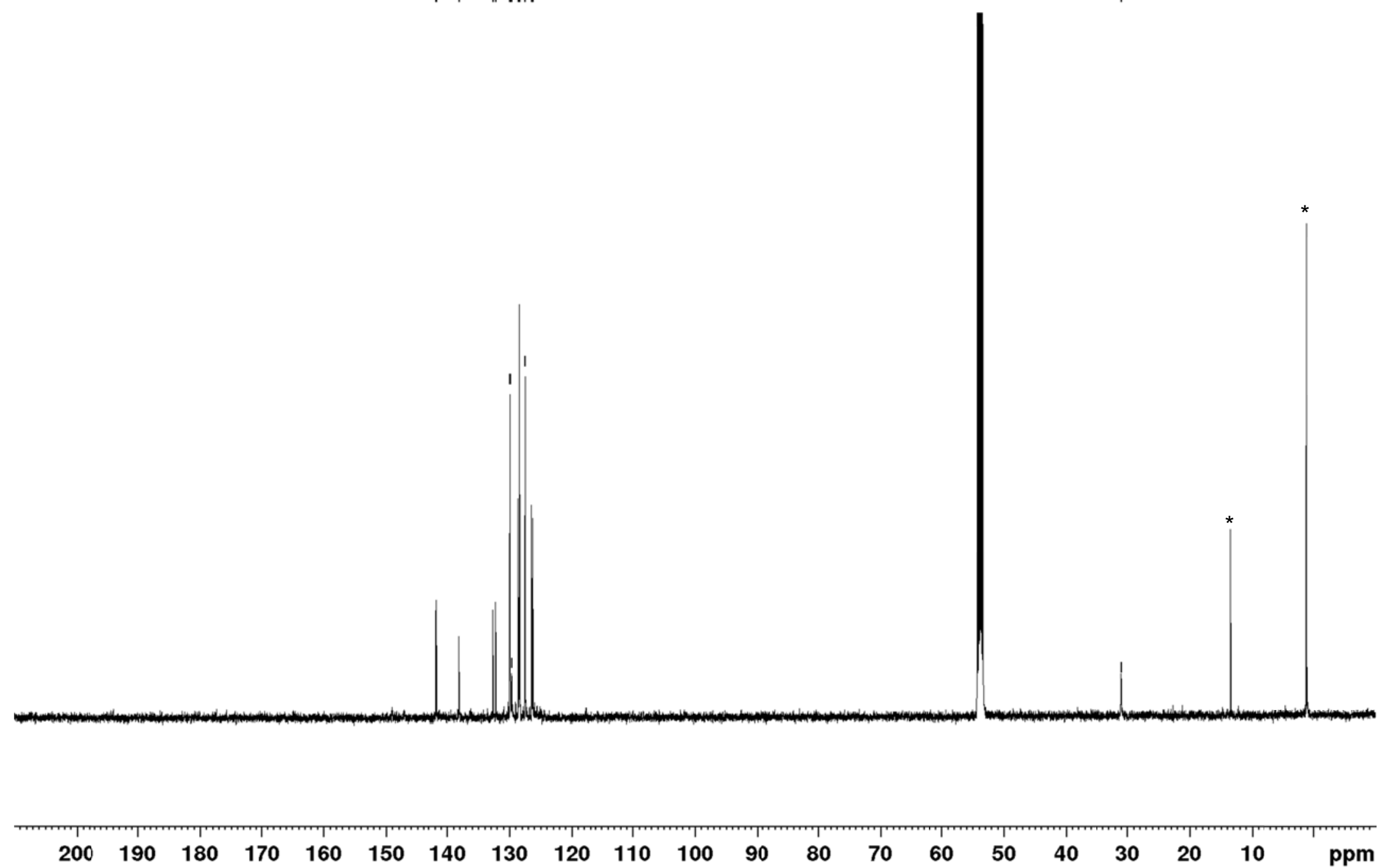


${ }^{11} \mathrm{~B} \operatorname{NMR}\left(161 \mathrm{MHz}, \mathrm{CD}_{2} \mathrm{Cl}_{2}\right)$ :

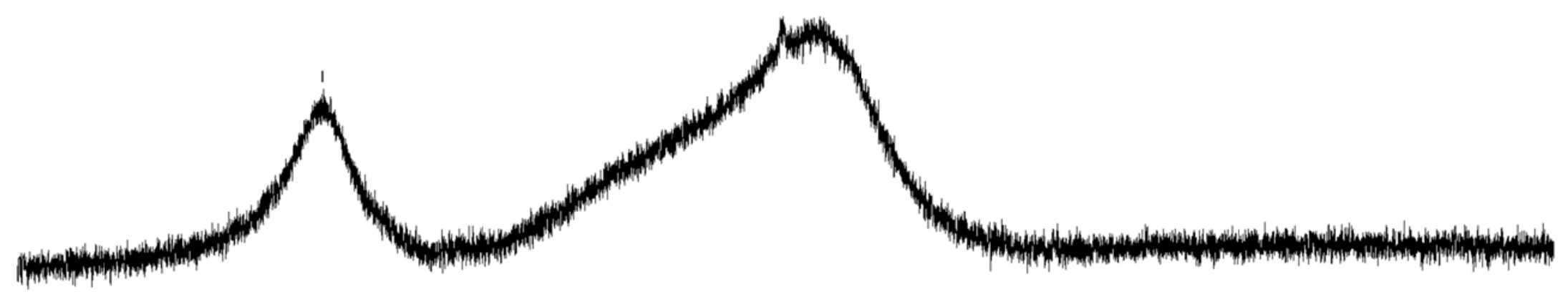

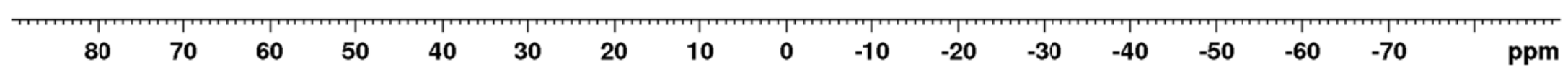


${ }^{19} \mathrm{~F}\left\{{ }^{1} \mathrm{H}\right\}$ NMR $\left(471 \mathrm{MHz}, \mathrm{CD}_{2} \mathrm{Cl}_{2}\right)$ :

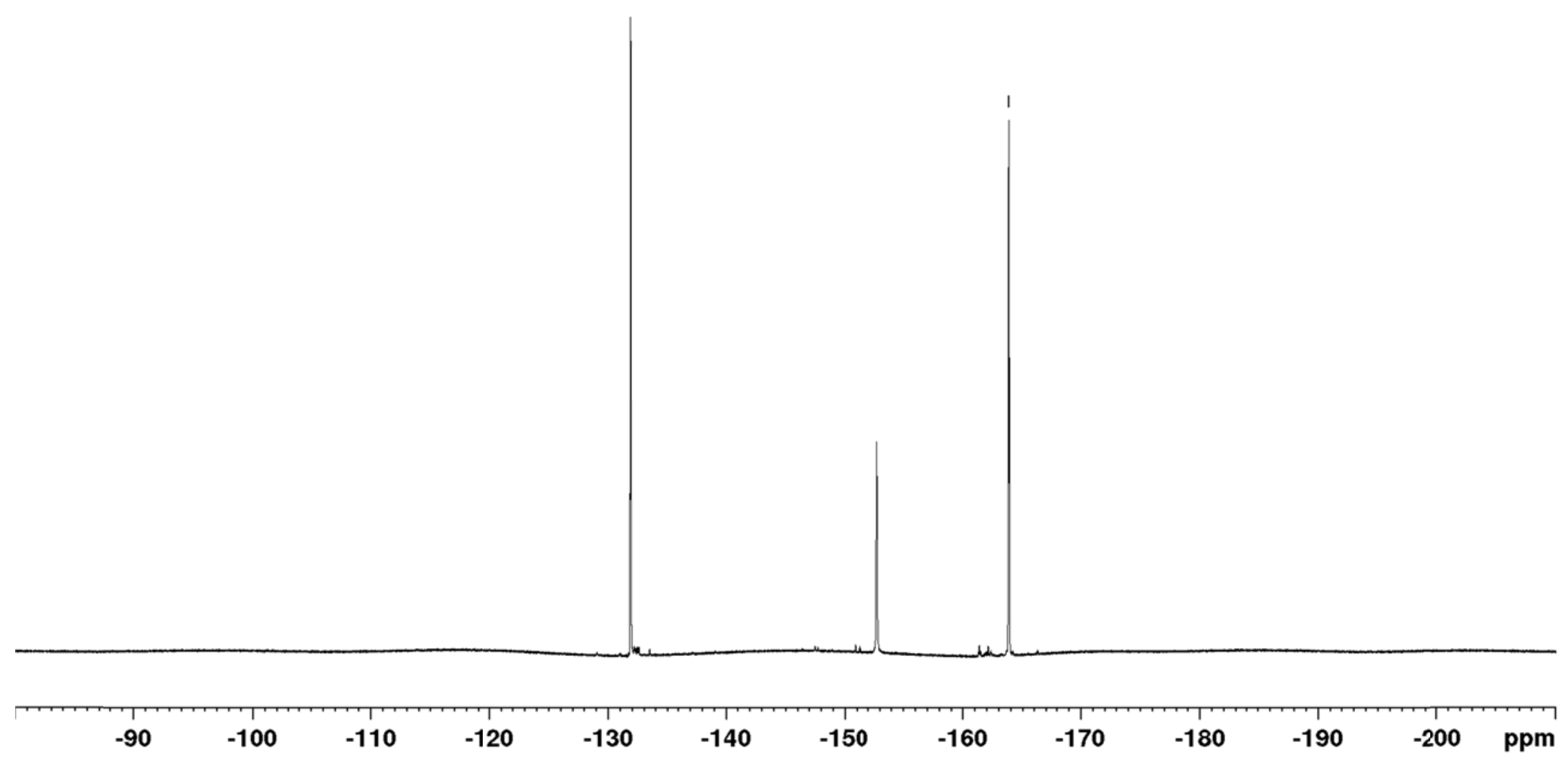


${ }^{19} \mathrm{~F} /{ }^{13} \mathrm{C}$ HMQC NMR (659/176 MHz, $\left.\mathrm{CD}_{2} \mathrm{Cl}_{2}\right):$

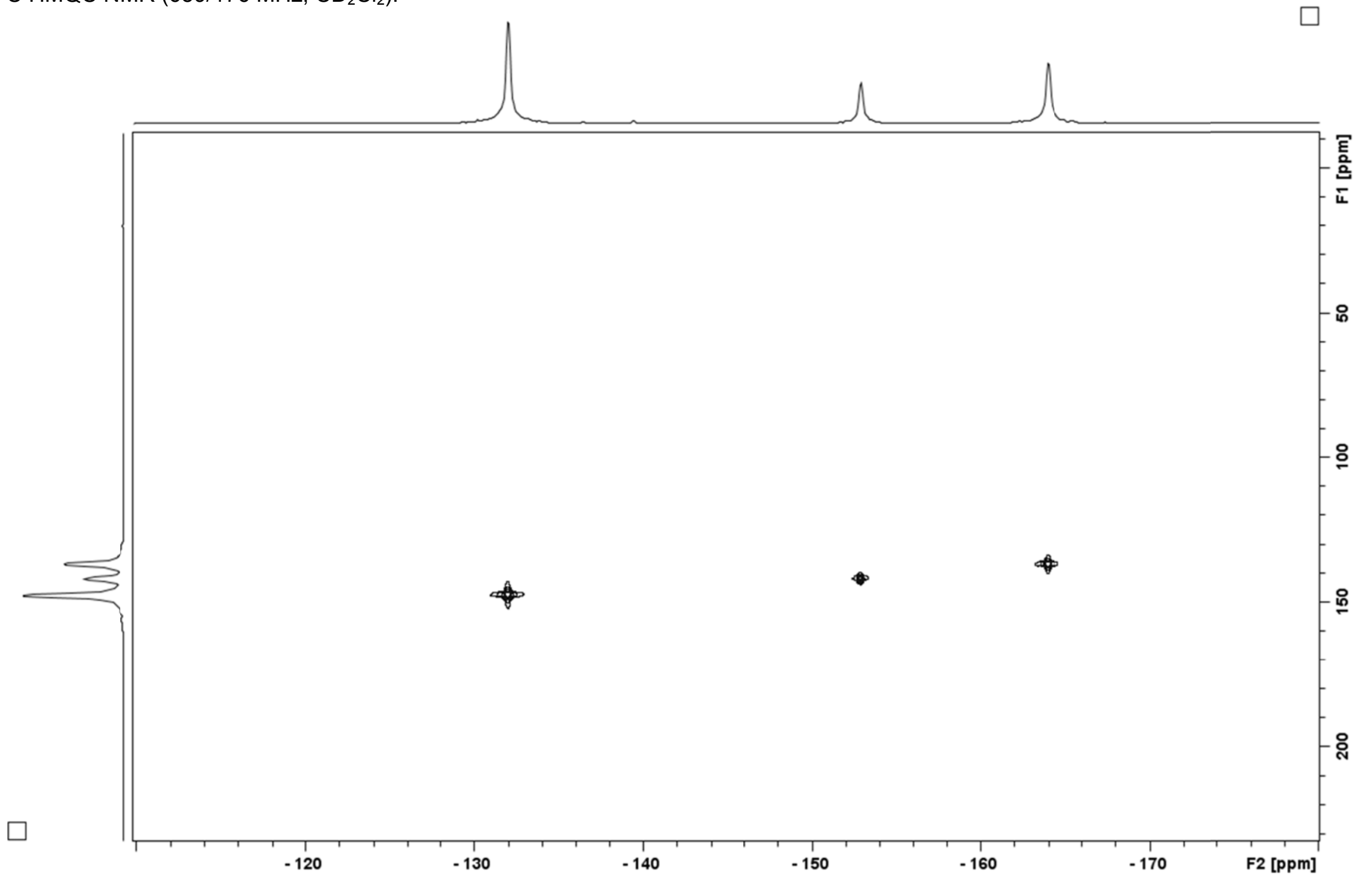


(S)-4-(Pentafluorophenyl)-4,5-dihydro-3H-(3,3'-diphenyl)dinaphtho[2,1-c:1',2'-e] borepine 3,3-Dimethyloxetane Adduct [(S)-3-3,3-DMO] ${ }^{1} \mathrm{H}$ NMR $\left(400 \mathrm{MHz}, \mathrm{CD}_{2} \mathrm{Cl}_{2}\right)$ :
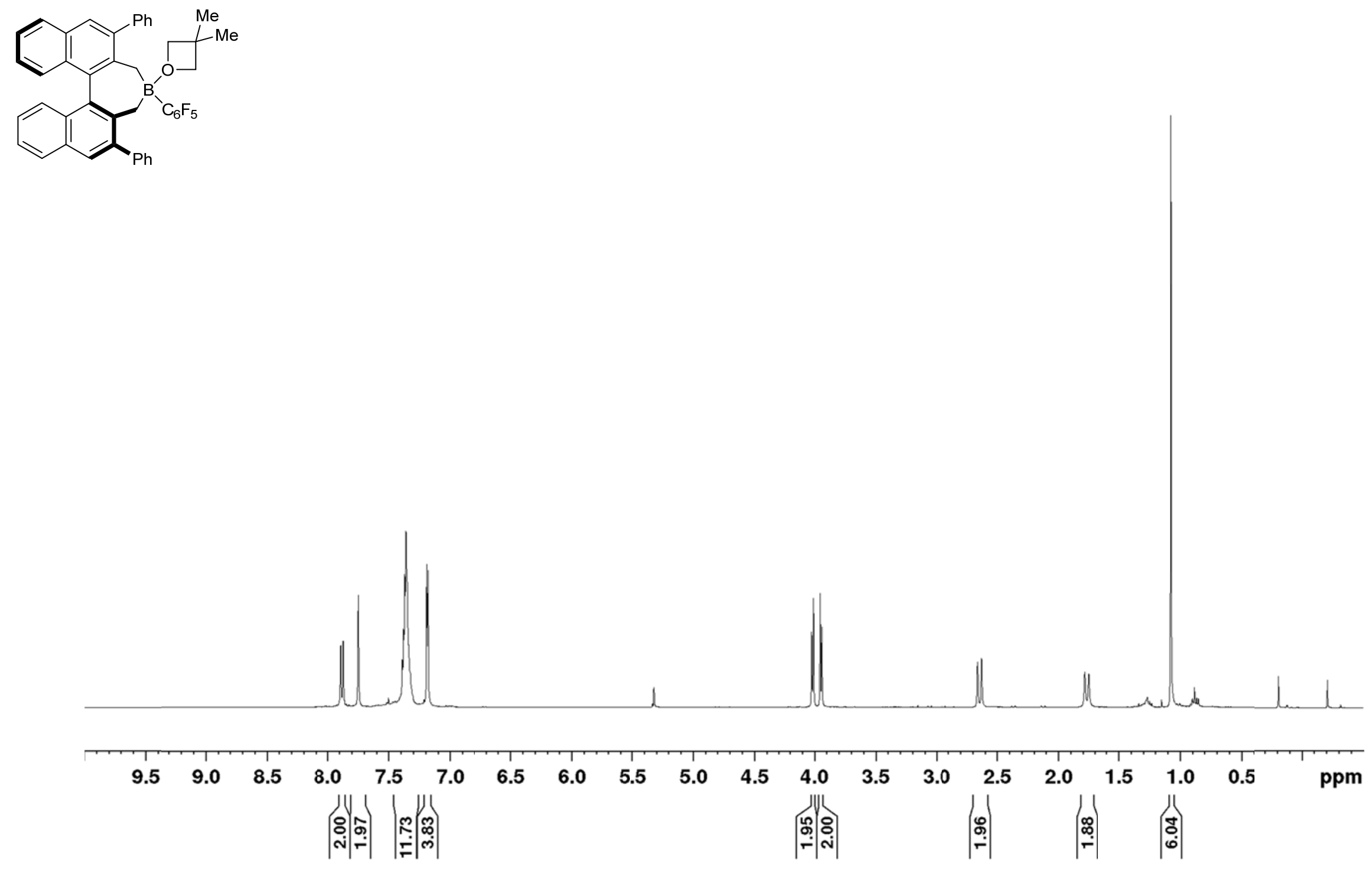
${ }^{13} \mathrm{C}\left\{{ }^{1} \mathrm{H}\right\}$ NMR $\left(126 \mathrm{MHz}, \mathrm{CD}_{2} \mathrm{Cl}_{2}\right):$
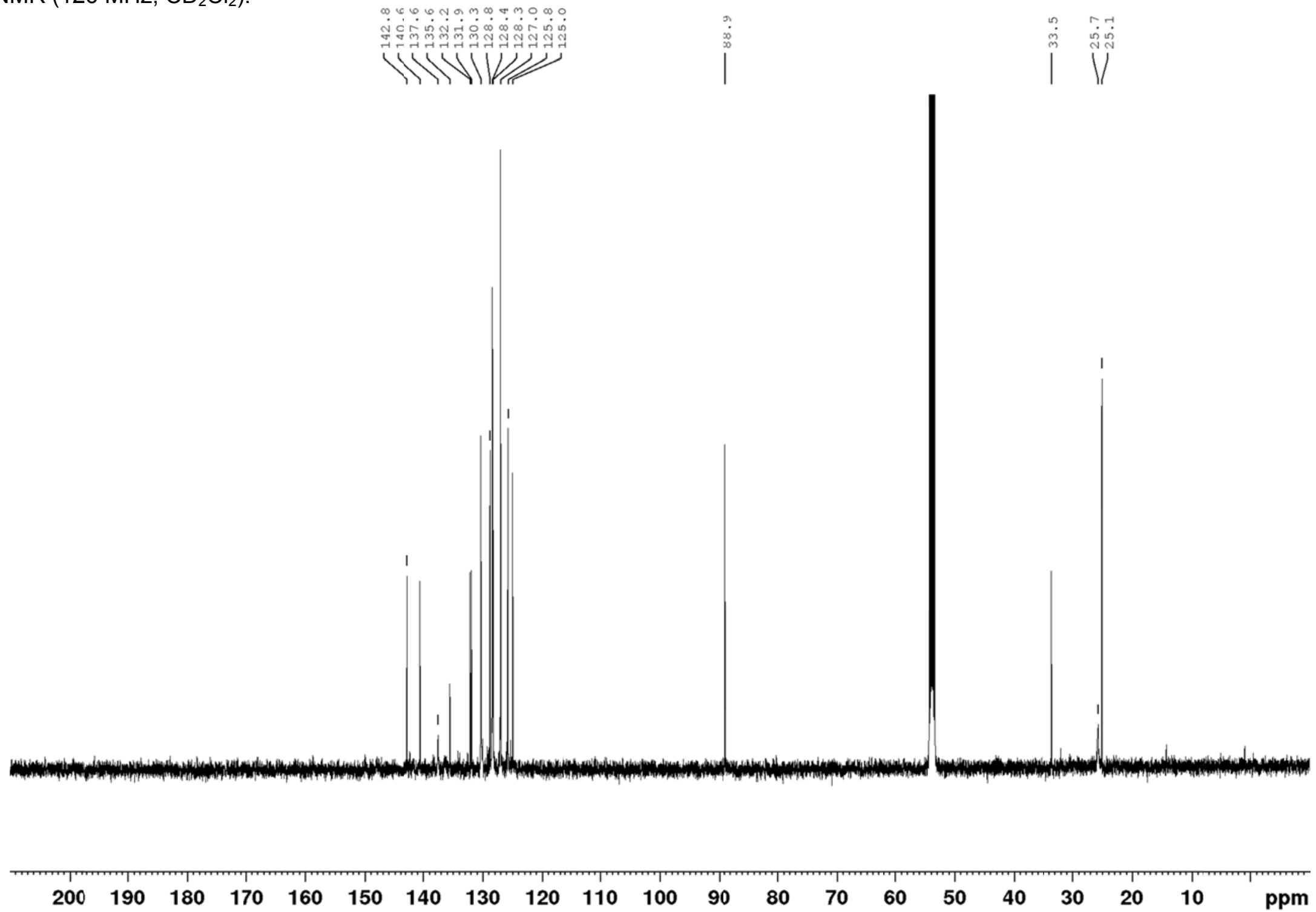
${ }^{11} \mathrm{~B}$ NMR $\left(161 \mathrm{MHz}, \mathrm{CD}_{2} \mathrm{Cl}_{2}\right)$ :

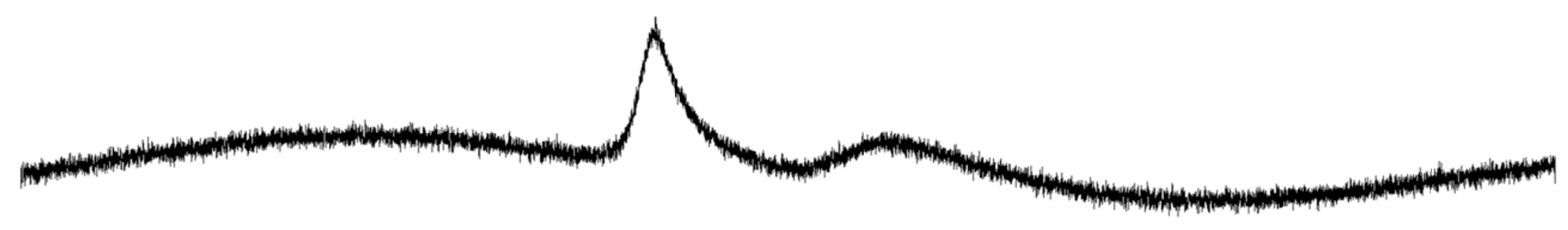

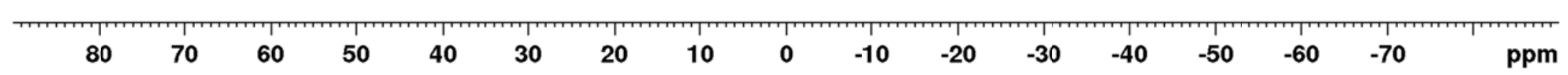


${ }^{19} \mathrm{~F}\left\{{ }^{1} \mathrm{H}\right\}$ NMR $\left(471 \mathrm{MHz}, \mathrm{CD}_{2} \mathrm{Cl}_{2}\right)$ :
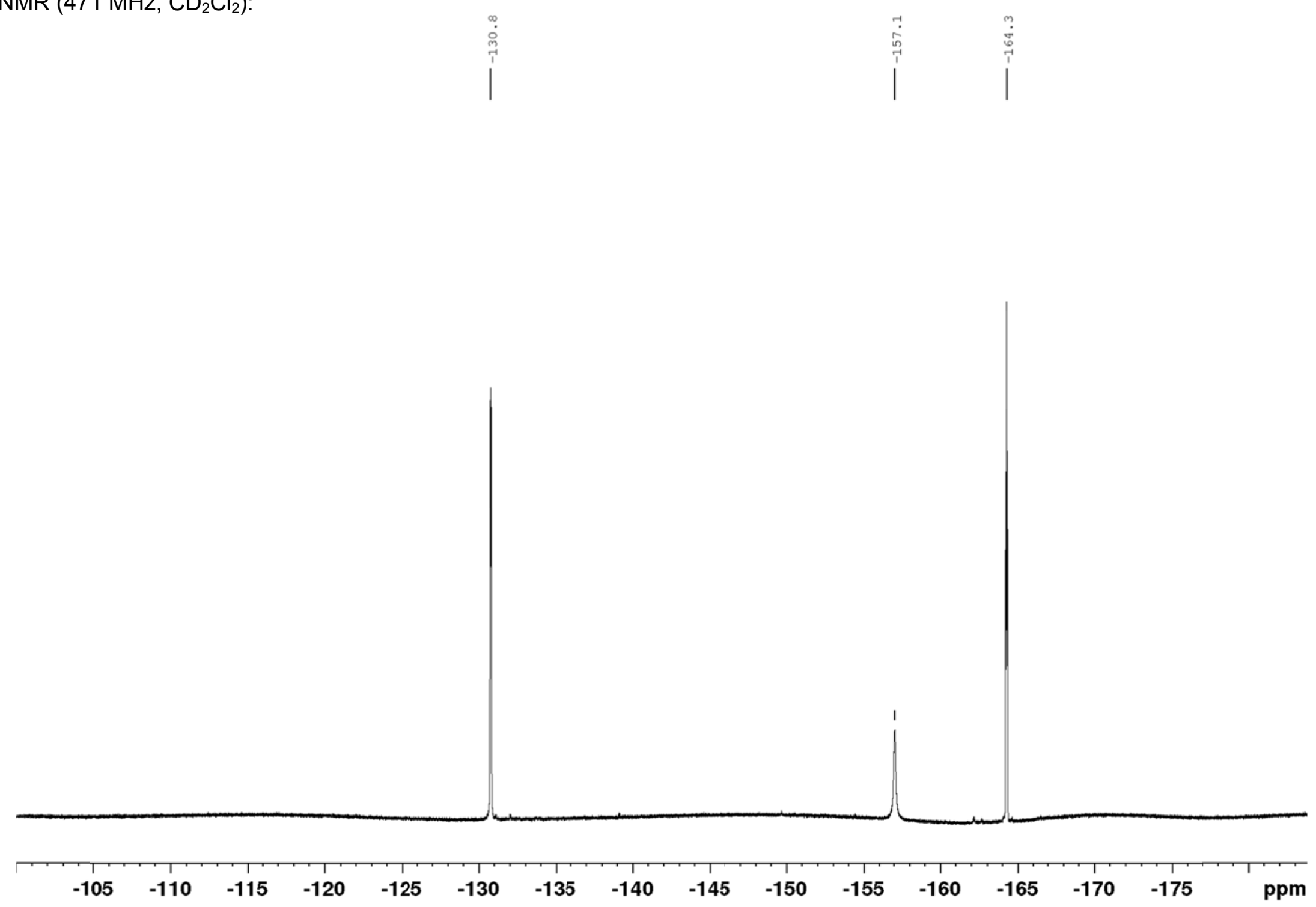
${ }^{19} \mathrm{~F} /{ }^{13} \mathrm{C}$ HMQC NMR (659/176 MHz, $\left.\mathrm{CD}_{2} \mathrm{Cl}_{2}\right)$ :

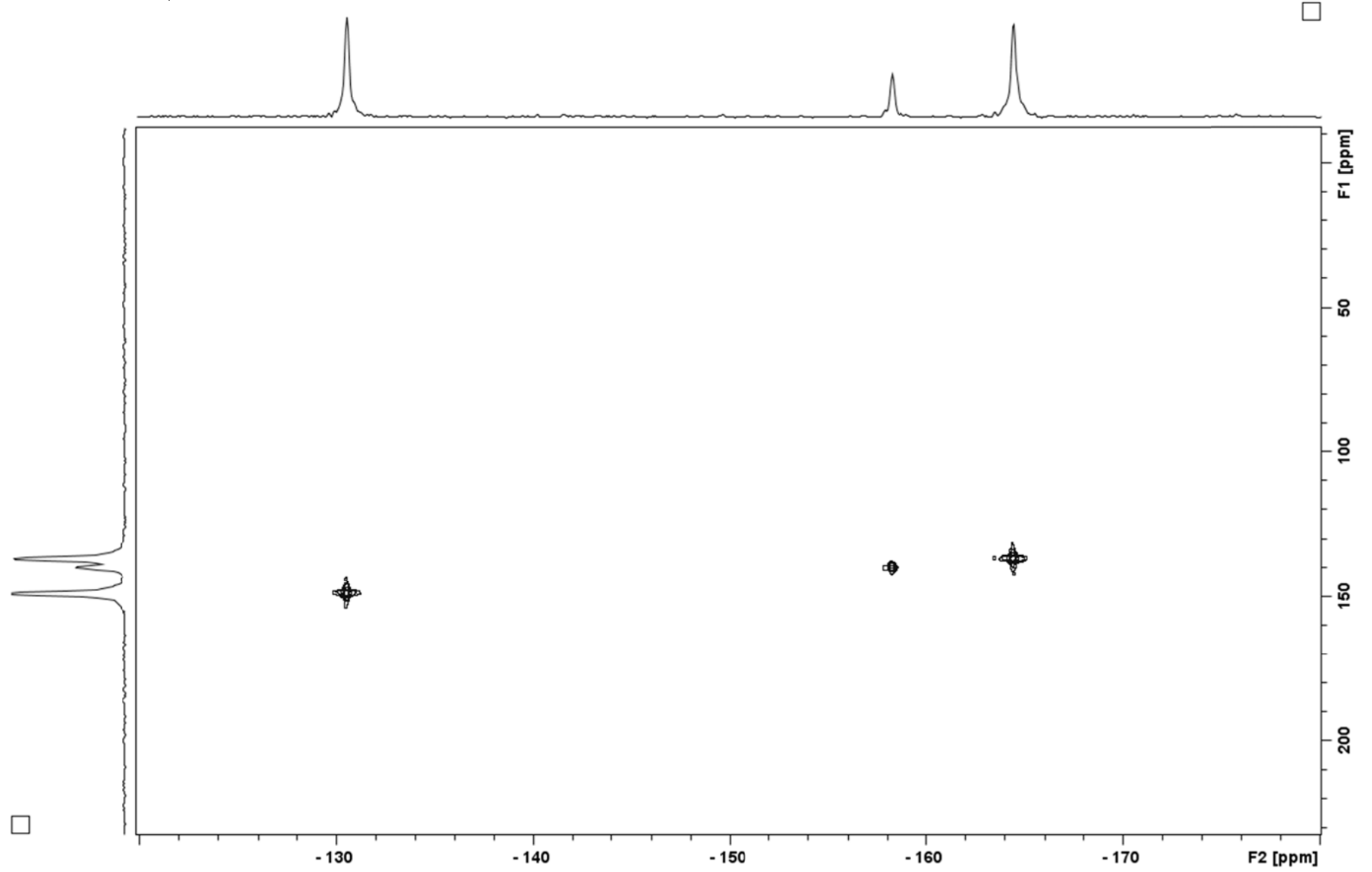


(S)-4-(Pentafluorophenyl)-4,5-dihydro-3H-(3,3'-diphenyl)dinaphtho[2,1-c:1',2'-e] borepine Dimethyl Sulfide Adduct [(S)-3·DMS] ${ }^{1} \mathrm{H}$ NMR $\left(700 \mathrm{MHz}, \mathrm{CD}_{2} \mathrm{Cl}_{2}\right)$ :
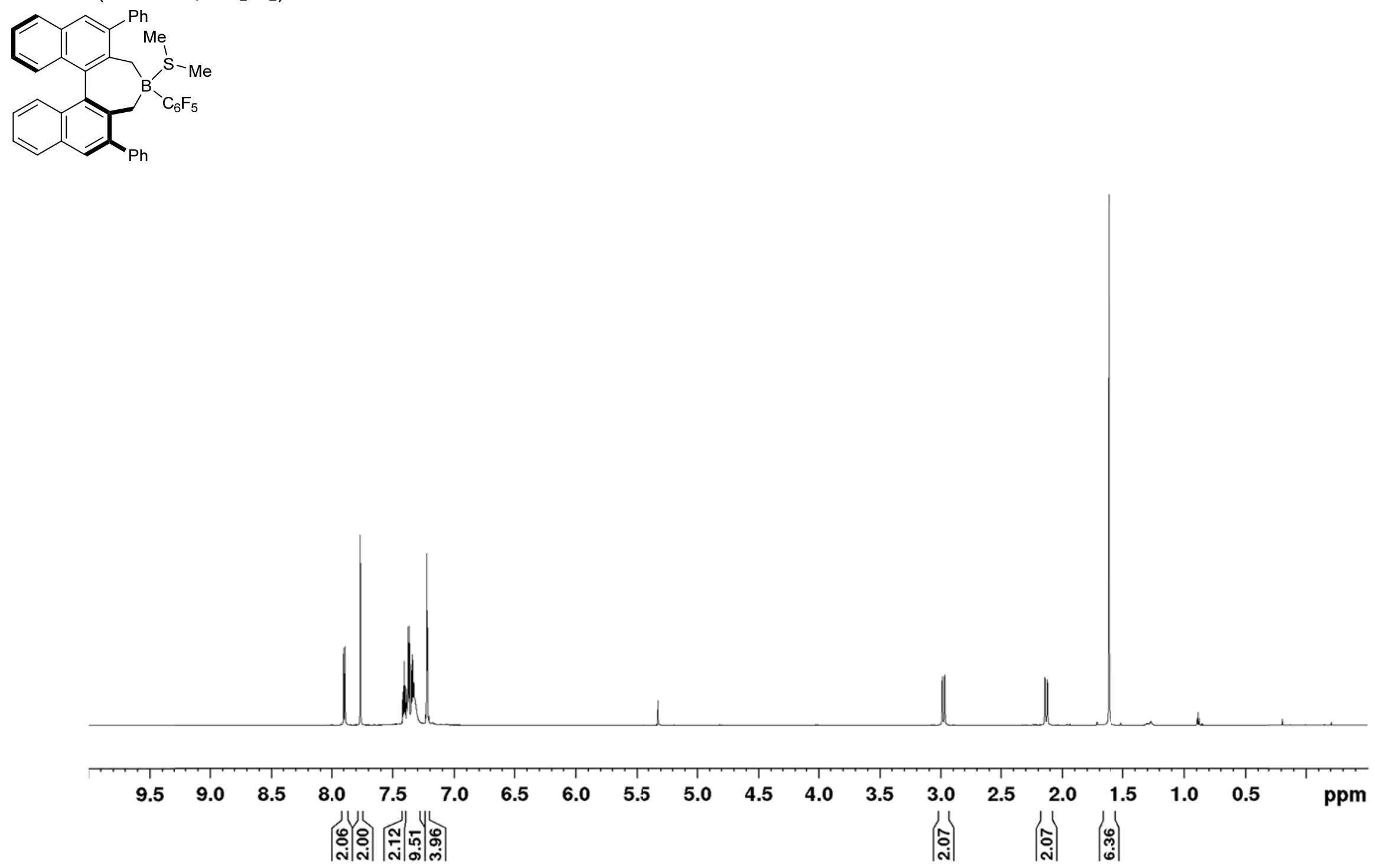


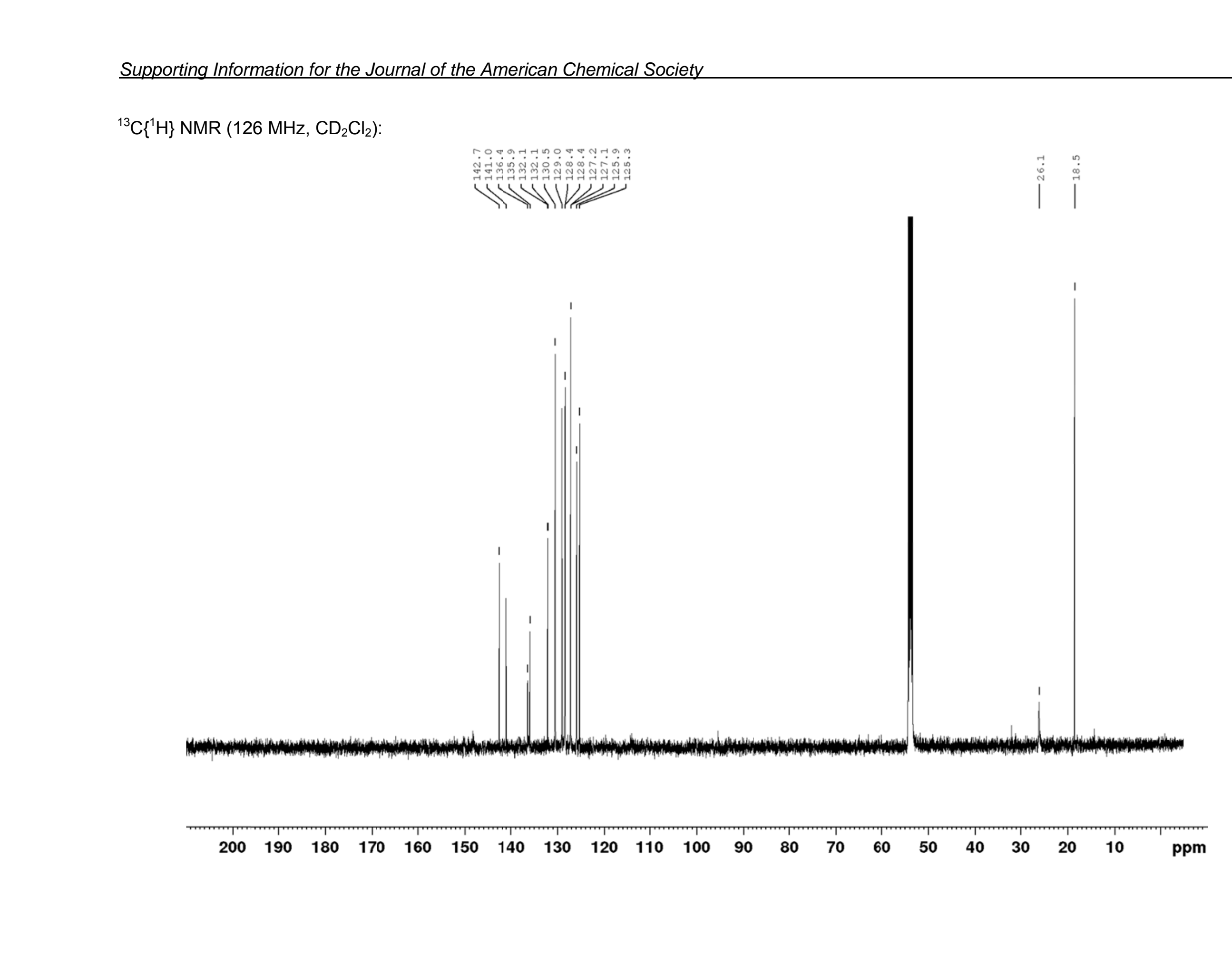


${ }^{11} \mathrm{~B} \operatorname{NMR}\left(161 \mathrm{MHz}, \mathrm{CD}_{2} \mathrm{Cl}_{2}\right)$ :

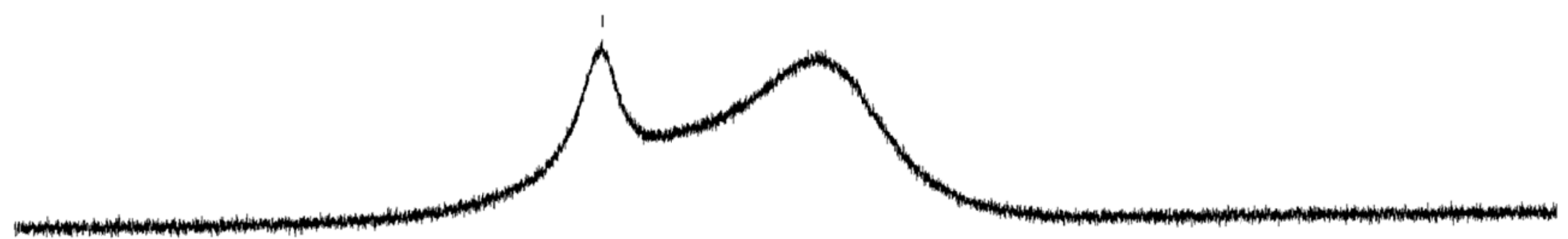

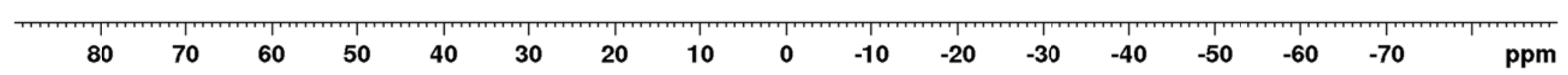


${ }^{19} \mathrm{~F}\left\{{ }^{1} \mathrm{H}\right\}$ NMR $\left(471 \mathrm{MHz}, \mathrm{CD}_{2} \mathrm{Cl}_{2}\right):$

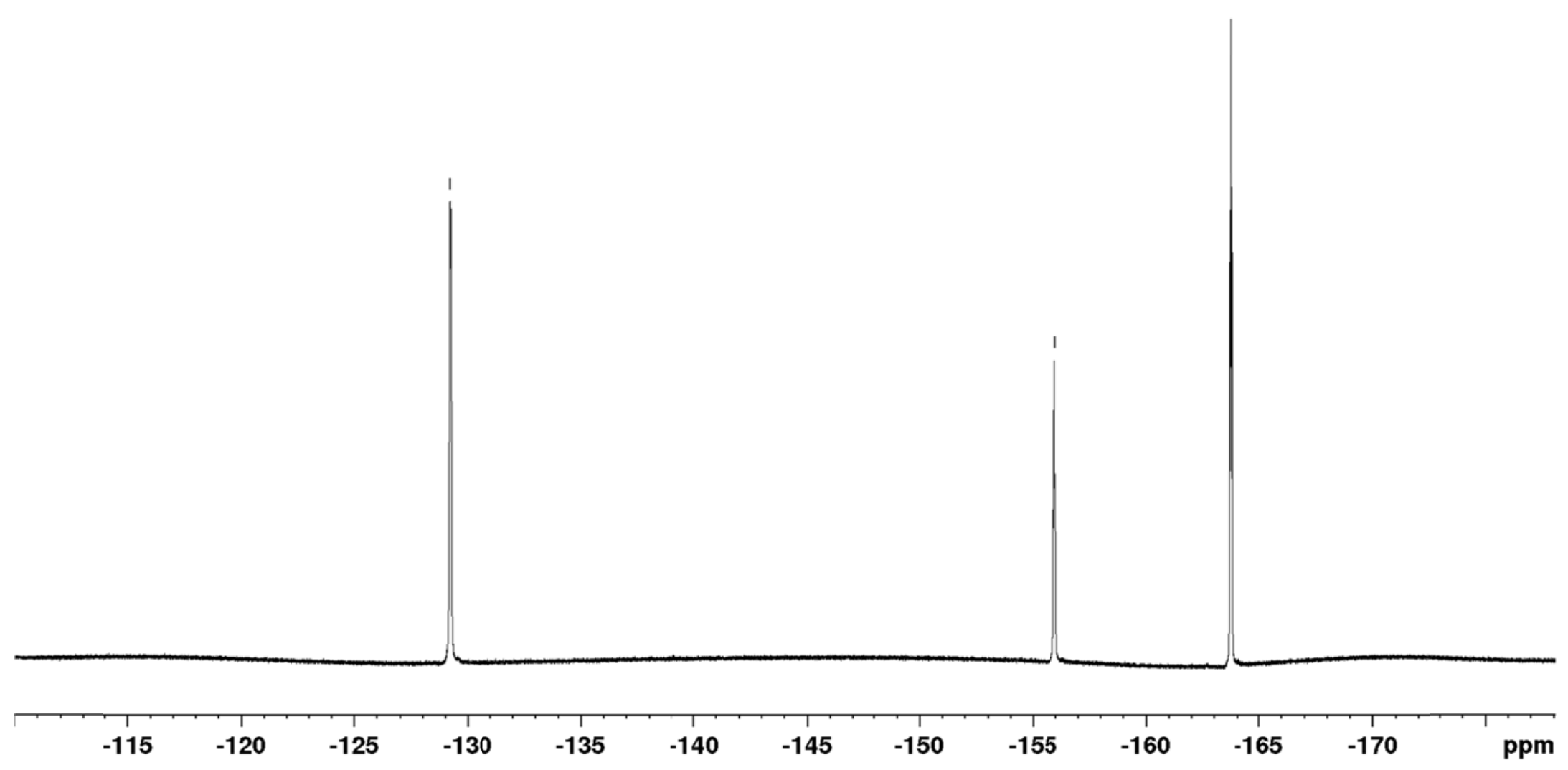


${ }^{19} \mathrm{~F} /{ }^{13} \mathrm{C}$ HMQC NMR (659/176 MHz, $\left.\mathrm{CD}_{2} \mathrm{Cl}_{2}\right)$ :

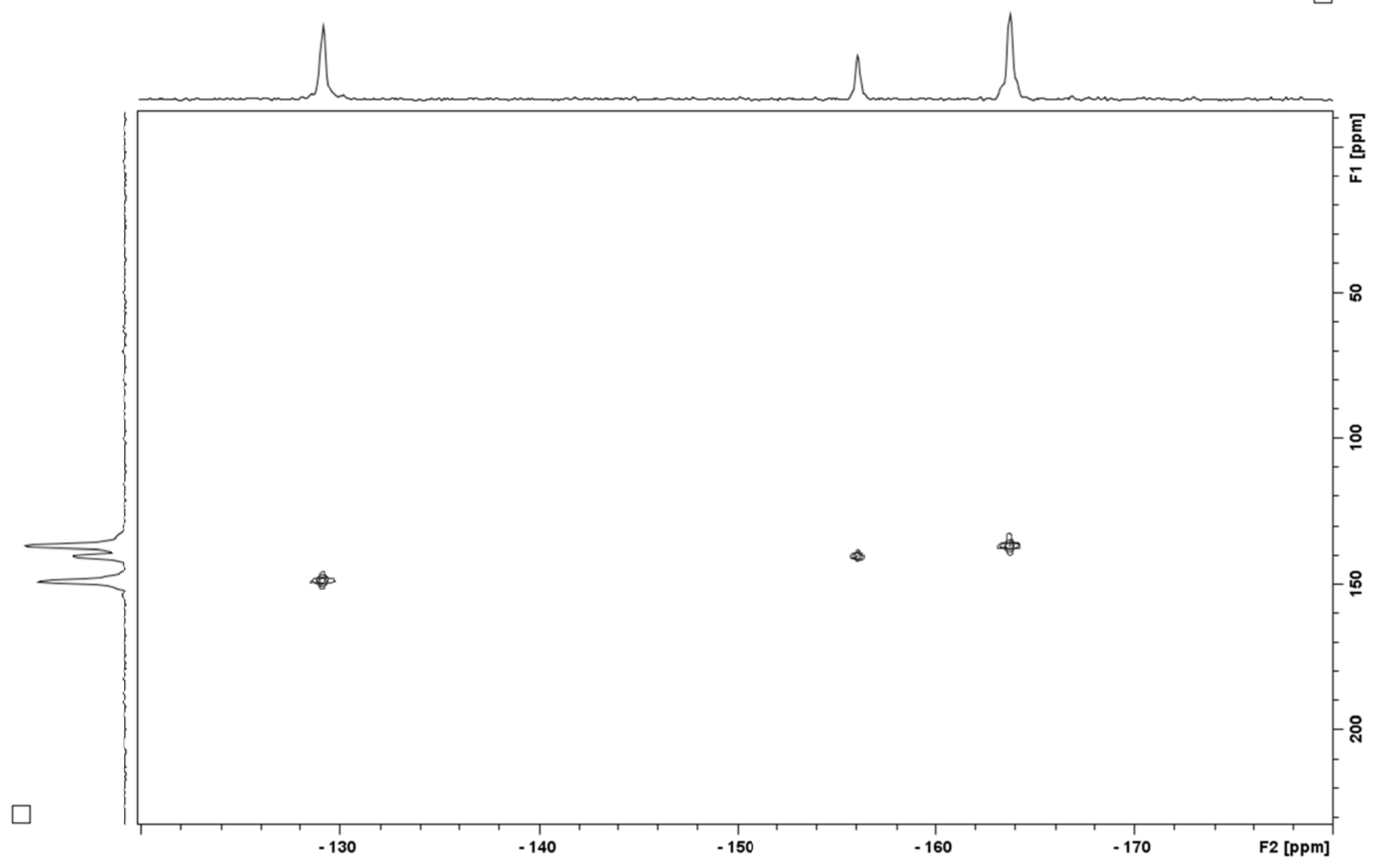




\section{(S)-1-Phenylethanol [(S)-7]}

${ }^{1} \mathrm{H}$ NMR (400 MHz, $\left.\mathrm{C}_{6} \mathrm{D}_{6}\right)$ :

${ }_{M e}^{O H}$

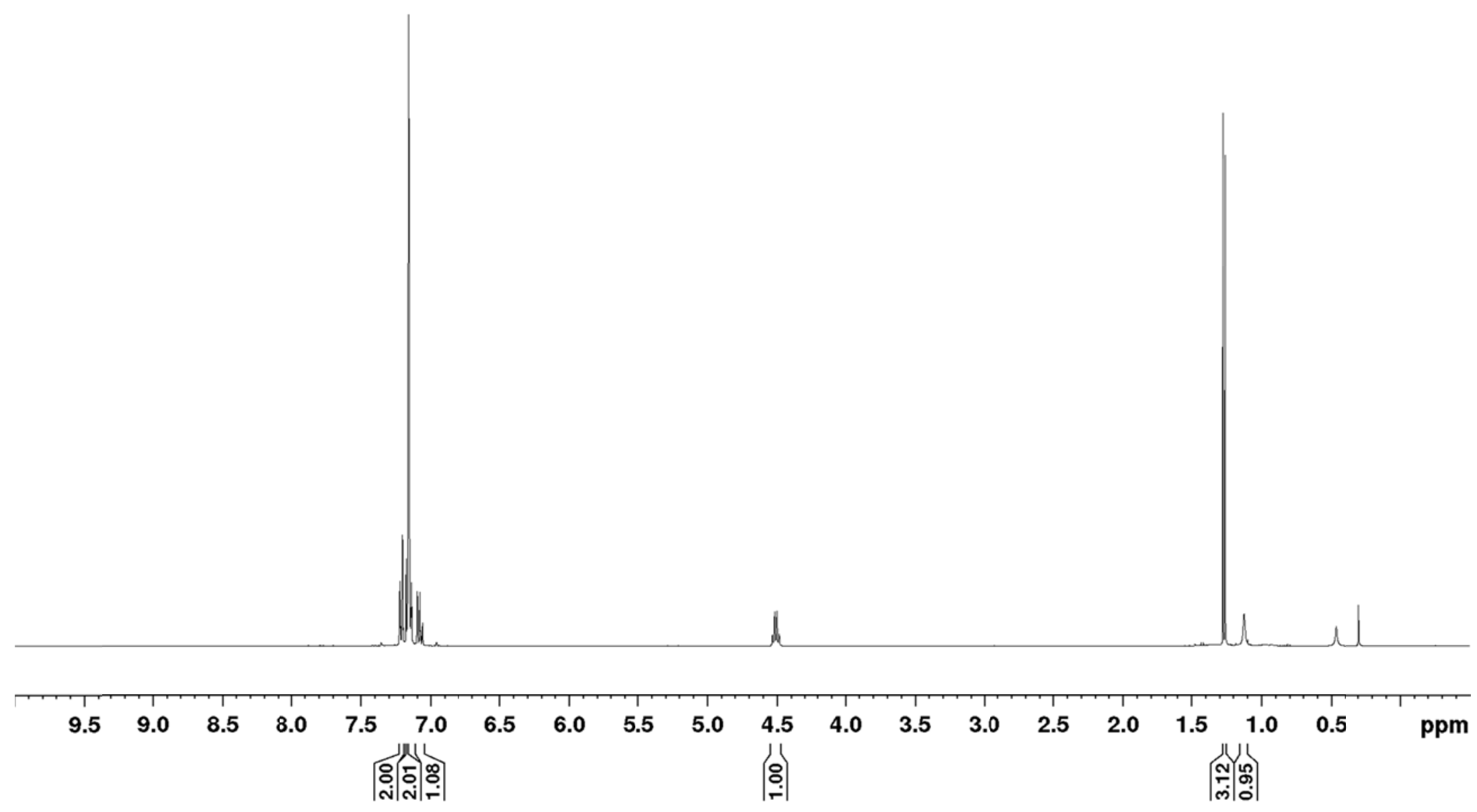


${ }^{13} \mathrm{C}\left\{{ }^{1} \mathrm{H}\right\} \operatorname{NMR}\left(101 \mathrm{MHz}, \mathrm{C}_{6} \mathrm{D}_{6}\right)$ :

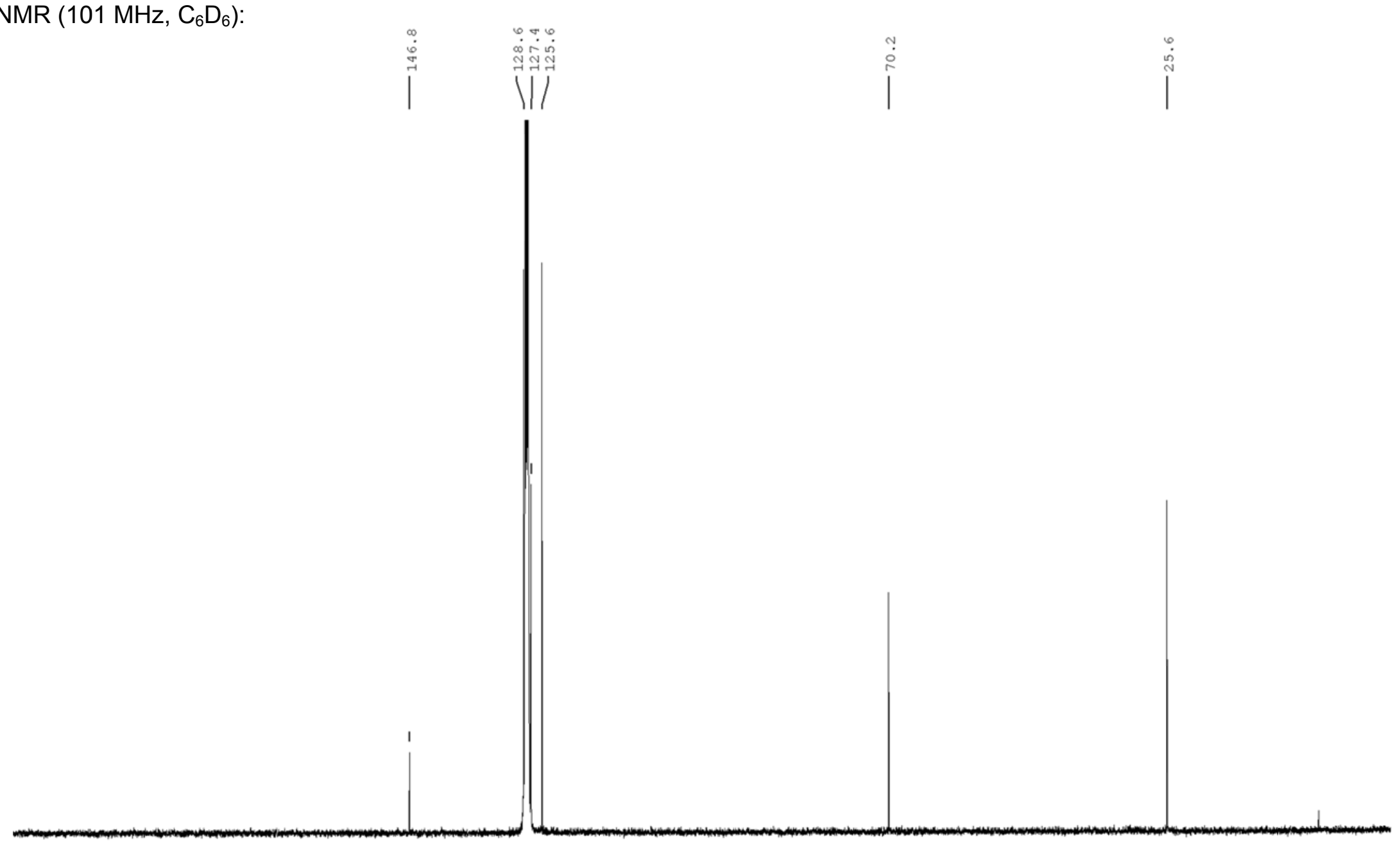

$\begin{array}{llllllllllllllllllllllll}200 & 190 & 180 & 170 & 160 & 150 & 140 & 130 & 120 & 110 & 100 & 90 & 80 & 70 & 60 & 50 & 40 & 30 & 20 & 10 & \text { ppm }\end{array}$ 


\section{(S)-1-(4'-Triflouromethylphenyl)ethanol [(S)-23]}

${ }^{1} \mathrm{H}$ NMR (500 MHz, $\left.\mathrm{CDCl}_{3}\right)$ :<smiles>COC(O)c1ccc(C(F)(F)F)cc1</smiles>

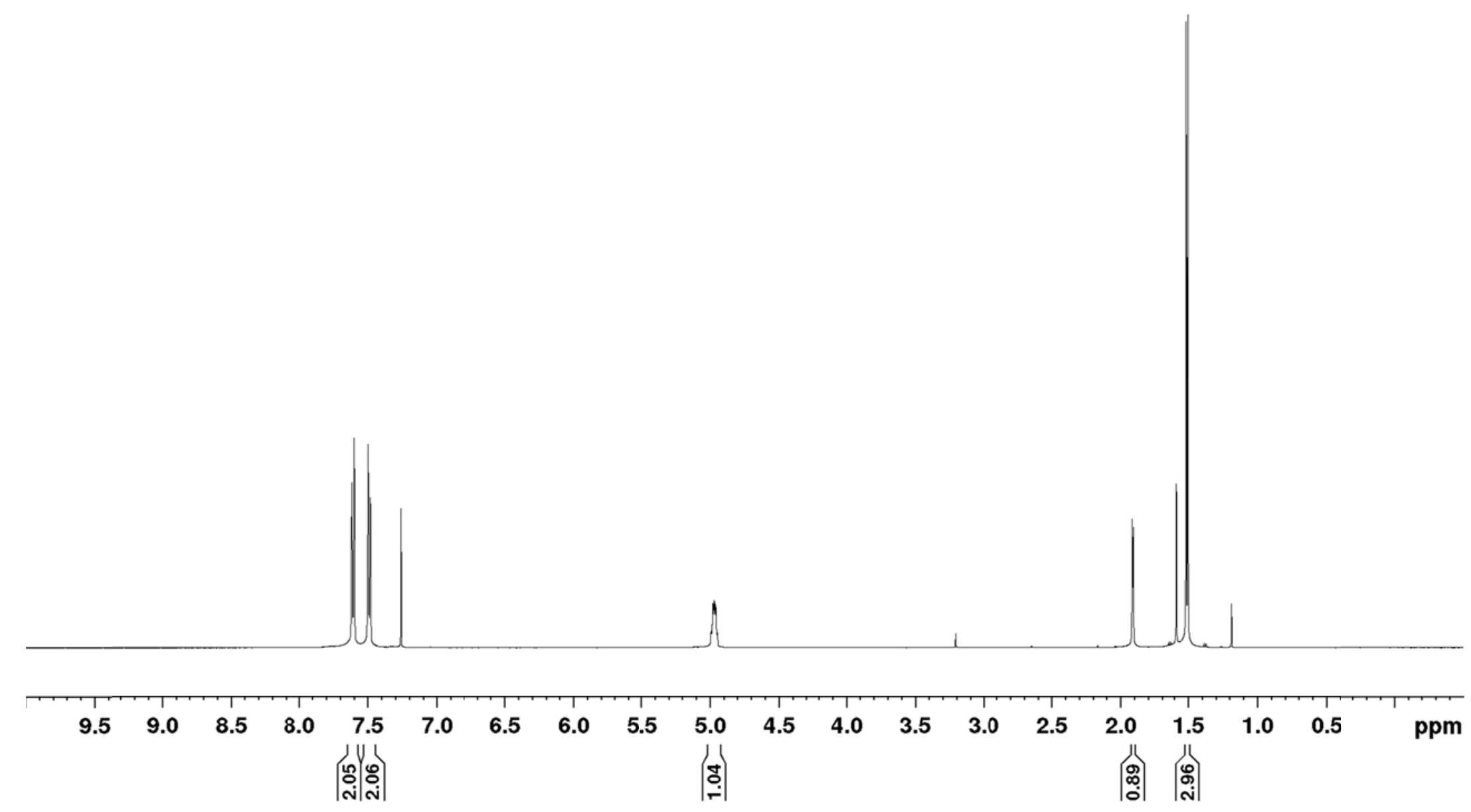


${ }^{13} \mathrm{C}\left\{{ }^{1} \mathrm{H}\right\} \operatorname{NMR}(126 \mathrm{MHz}, \mathrm{CDCl} 3):$

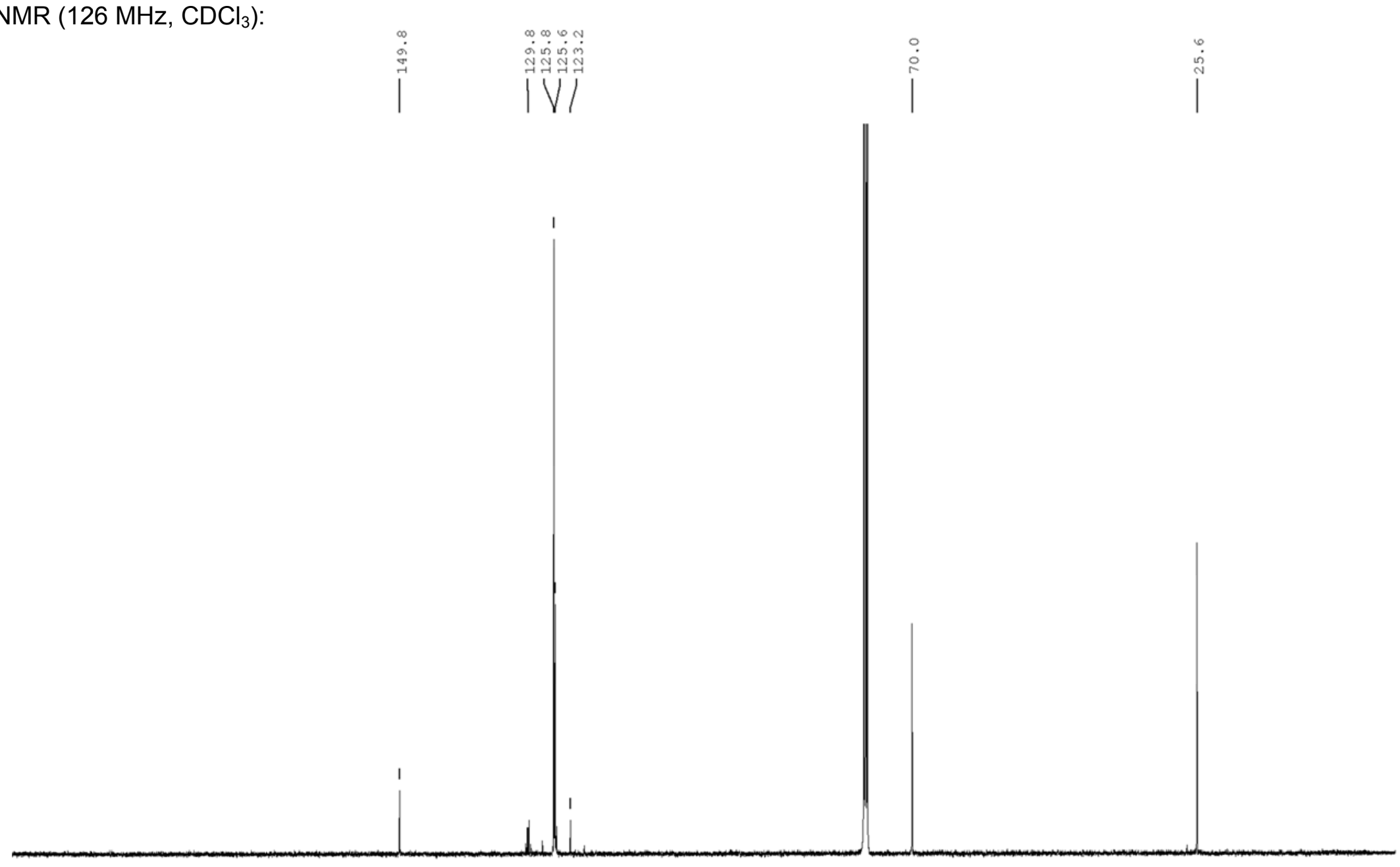

$\begin{array}{lllllllllllllllllllll}200 & 190 & 180 & 170 & 160 & 150 & 140 & 130 & 120 & 110 & 100 & 90 & 80 & 70 & 60 & 50 & 40 & 30 & 20 & 10 & \mathrm{ppm}\end{array}$ 
${ }^{19} \mathrm{~F}\left\{{ }^{1} \mathrm{H}\right\}$ NMR $\left(471 \mathrm{MHz}, \mathrm{CDCl}_{3}\right):$

$$
\text { | }
$$

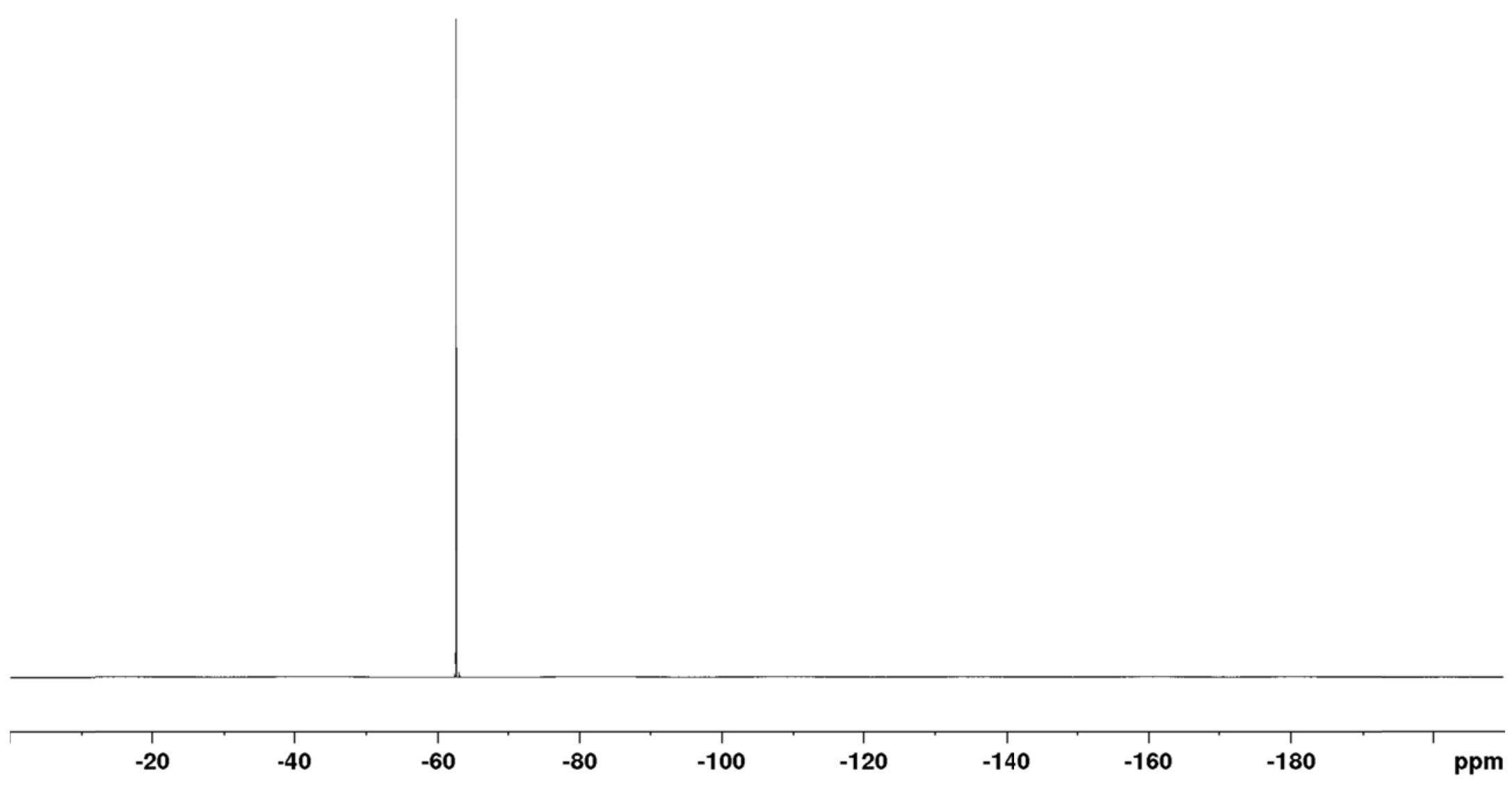




\section{(S)-1-(4'-Nitrophenyl)ethanol [(S)-24]}

${ }^{1} \mathrm{H}$ NMR (400 MHz, $\left.\mathrm{CDCl}_{3}\right)$ :
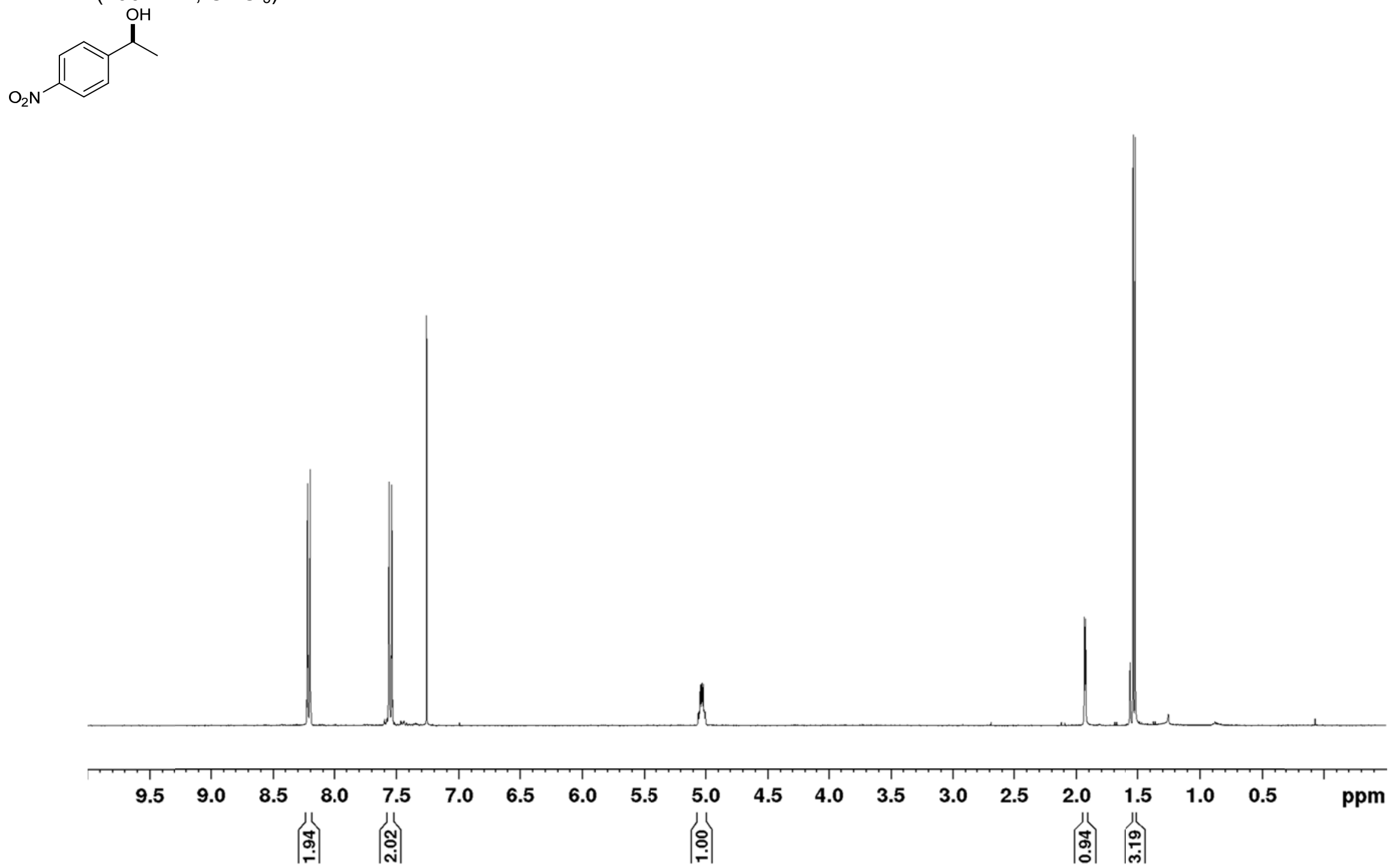
${ }^{13} \mathrm{C}\left\{{ }^{1} \mathrm{H}\right\}$ NMR $\left(101 \mathrm{MHz}, \mathrm{CDCl}_{3}\right):$
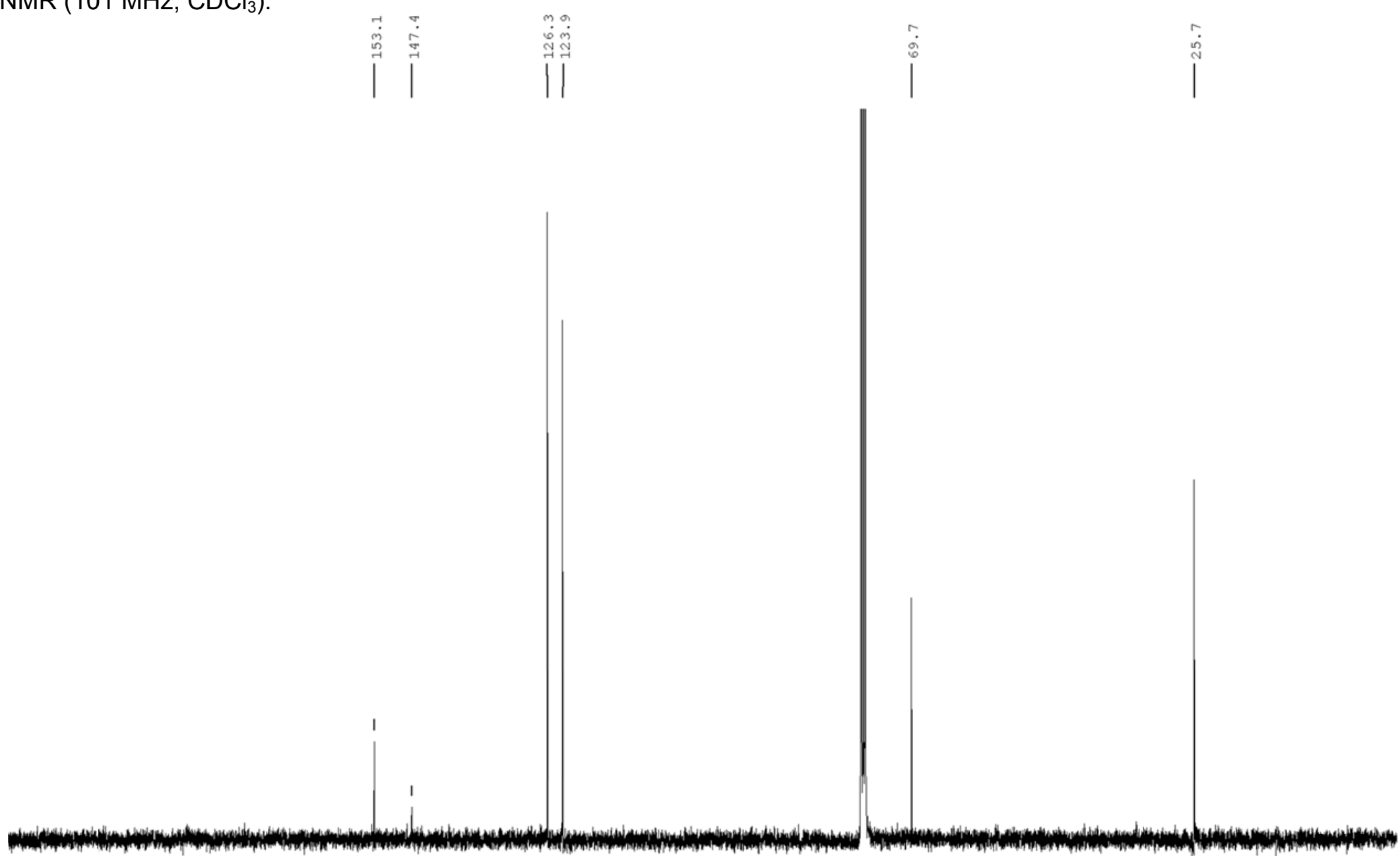
200190
$\begin{array}{llll}180 & 170 & 160 & 150\end{array}$
$\begin{array}{lll}140 \quad 130 & 120\end{array}$
110100
70

9080

60

$\begin{array}{llllll}40 & 30 & 20 & 10 & \mathrm{ppm}\end{array}$




\section{(S)-Methyl-4-(1-hydroxyethyl)benzoate [(S)-25]}

${ }^{1} \mathrm{H}$ NMR $\left(400 \mathrm{MHz}, \mathrm{CDCl}_{3}\right)$ :<smiles>CC(=O)c1ccc(C(C)O)cc1</smiles>

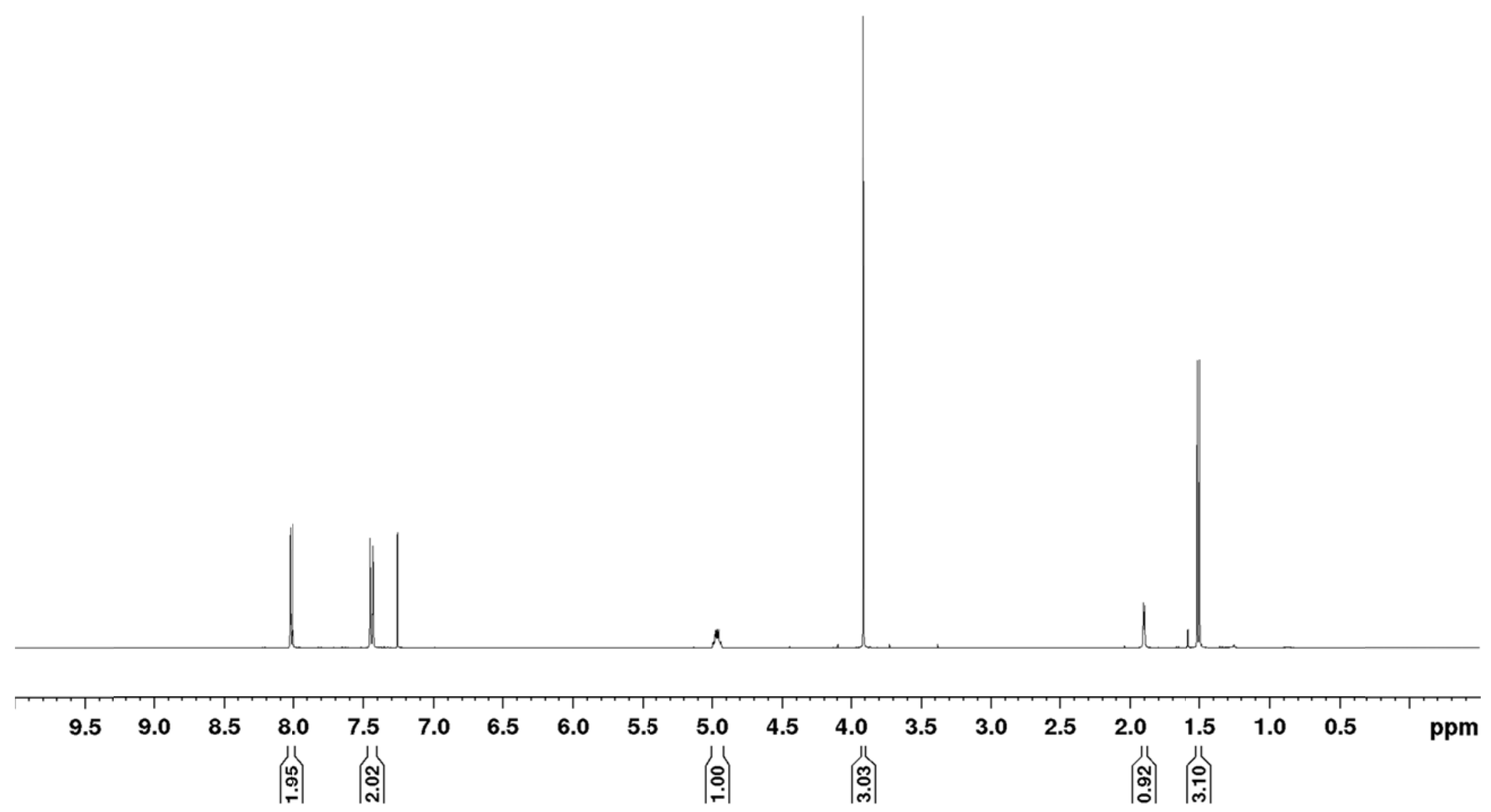


${ }^{13} \mathrm{C}\left\{{ }^{1} \mathrm{H}\right\}$ NMR $\left(101 \mathrm{MHz}, \mathrm{CDCl}_{3}\right):$
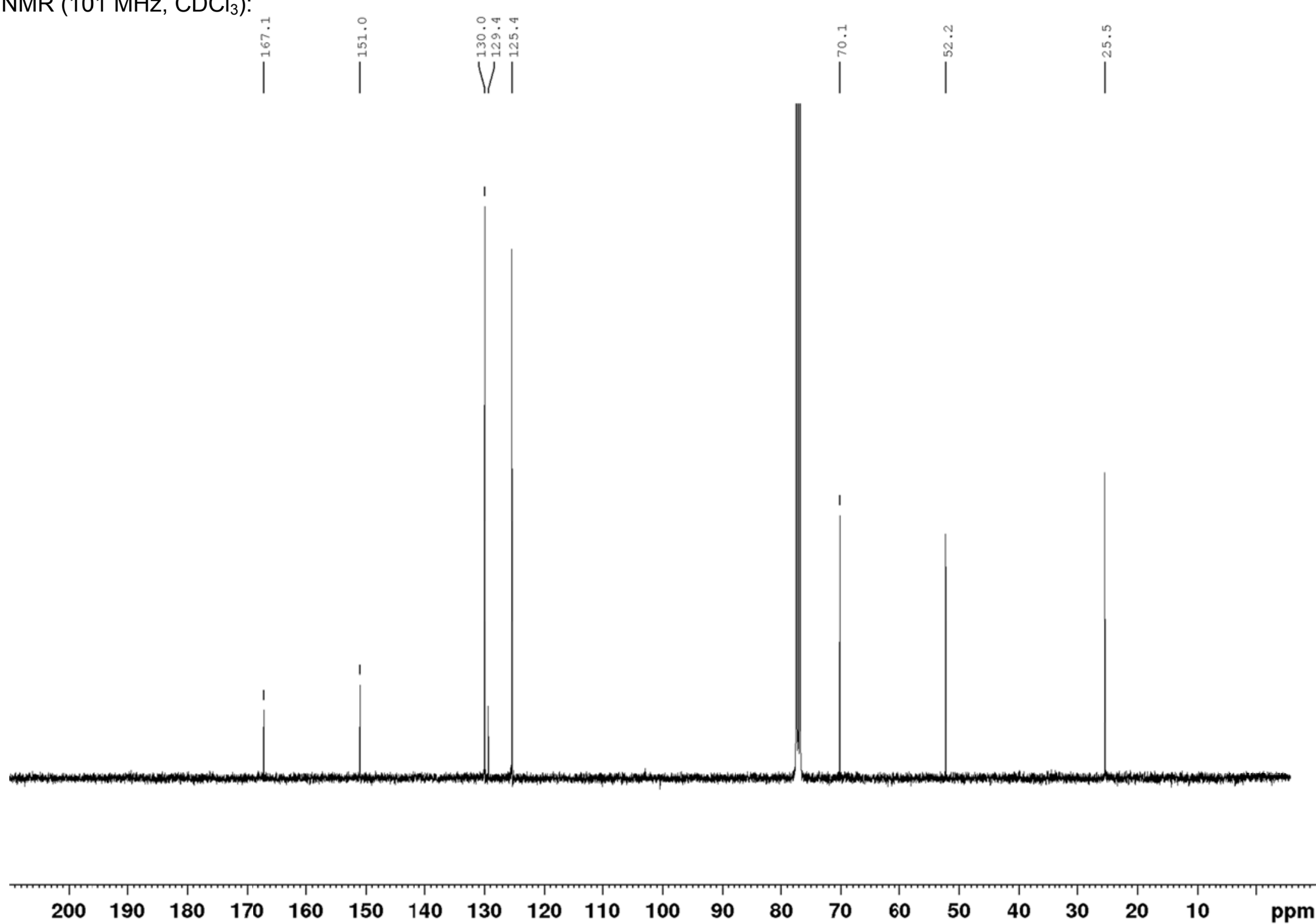


\section{(S)-1-(4'-Chlorophenyl)ethanol [(S)-26]}

${ }^{1} \mathrm{H}$ NMR (400 MHz, $\mathrm{C}_{6} \mathrm{D}_{6}$ ):
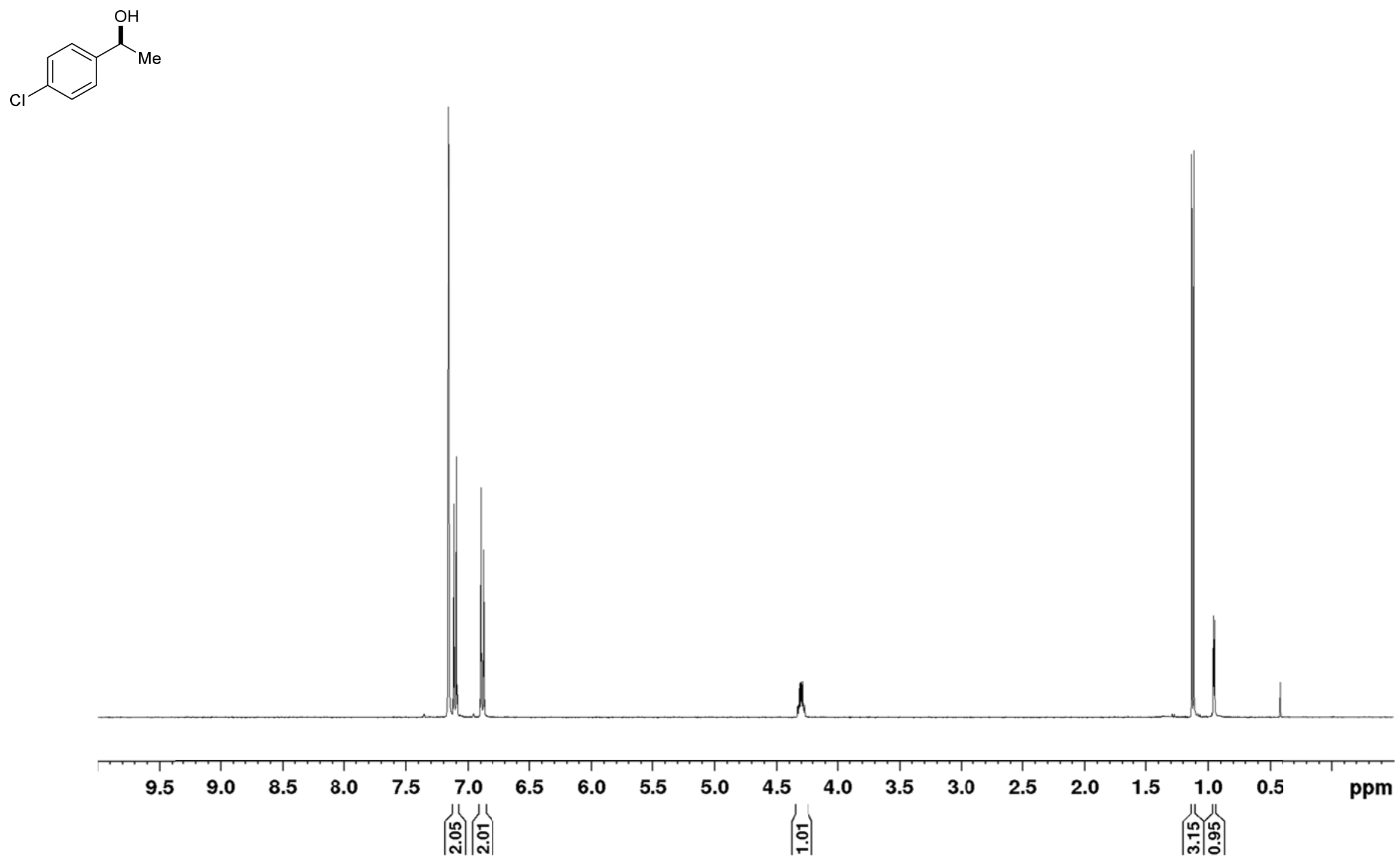
${ }^{13} \mathrm{C}\left\{{ }^{1} \mathrm{H}\right\} \operatorname{NMR}\left(101 \mathrm{MHz}, \mathrm{C}_{6} \mathrm{D}_{6}\right):$

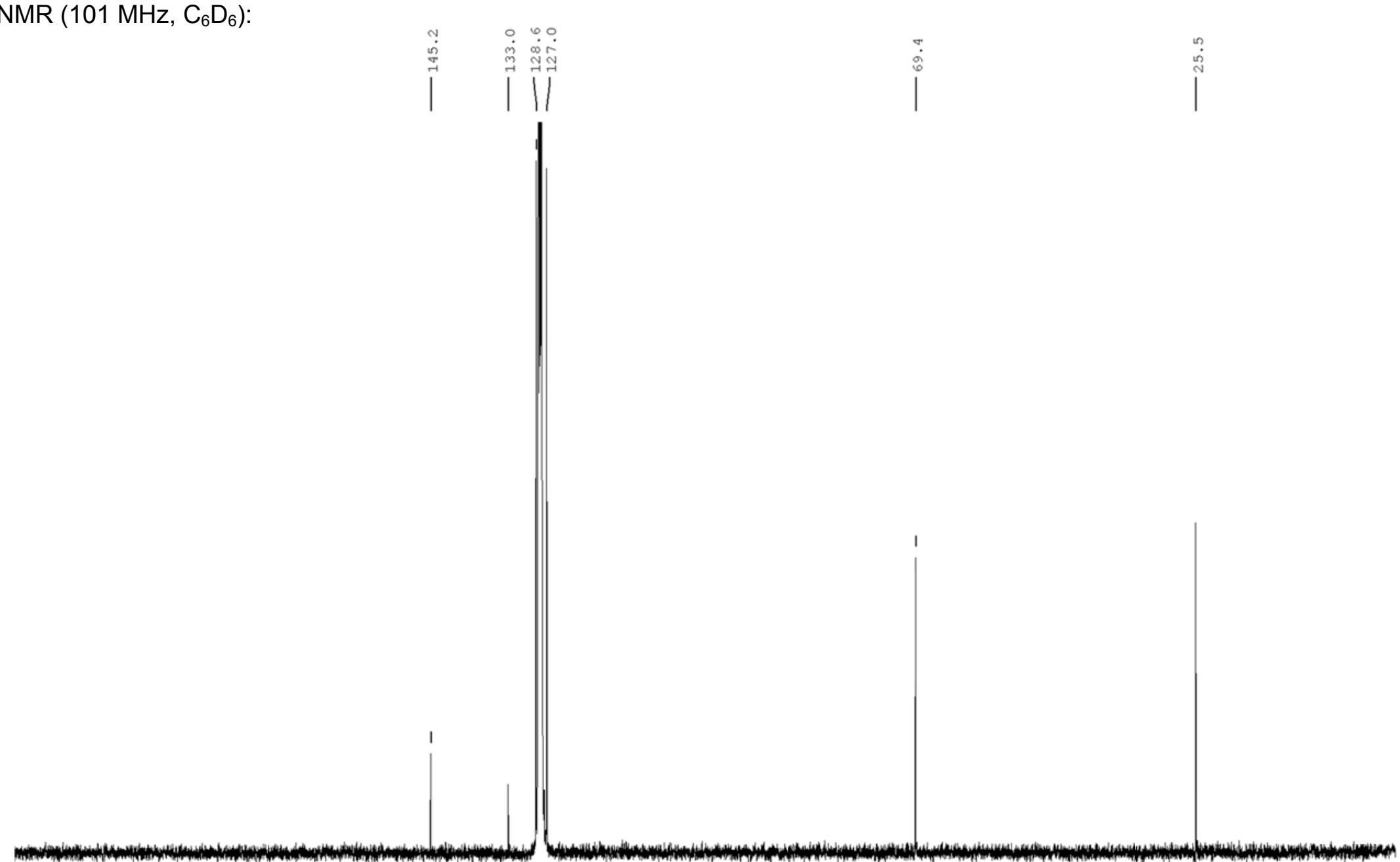

$\begin{array}{lllllllll}200 & 190 & 180 & 170 & 160 & 150 & 140 & 130 & 1\end{array}$ 


\section{(S)-1-(4'-Methylphenyl)ethanol [(S)-27]}

${ }^{1} \mathrm{H}$ NMR (400 MHz, $\left.\mathrm{CDCl}_{3}\right)$ :<smiles>Cc1ccc(C(O)O)cc1</smiles>

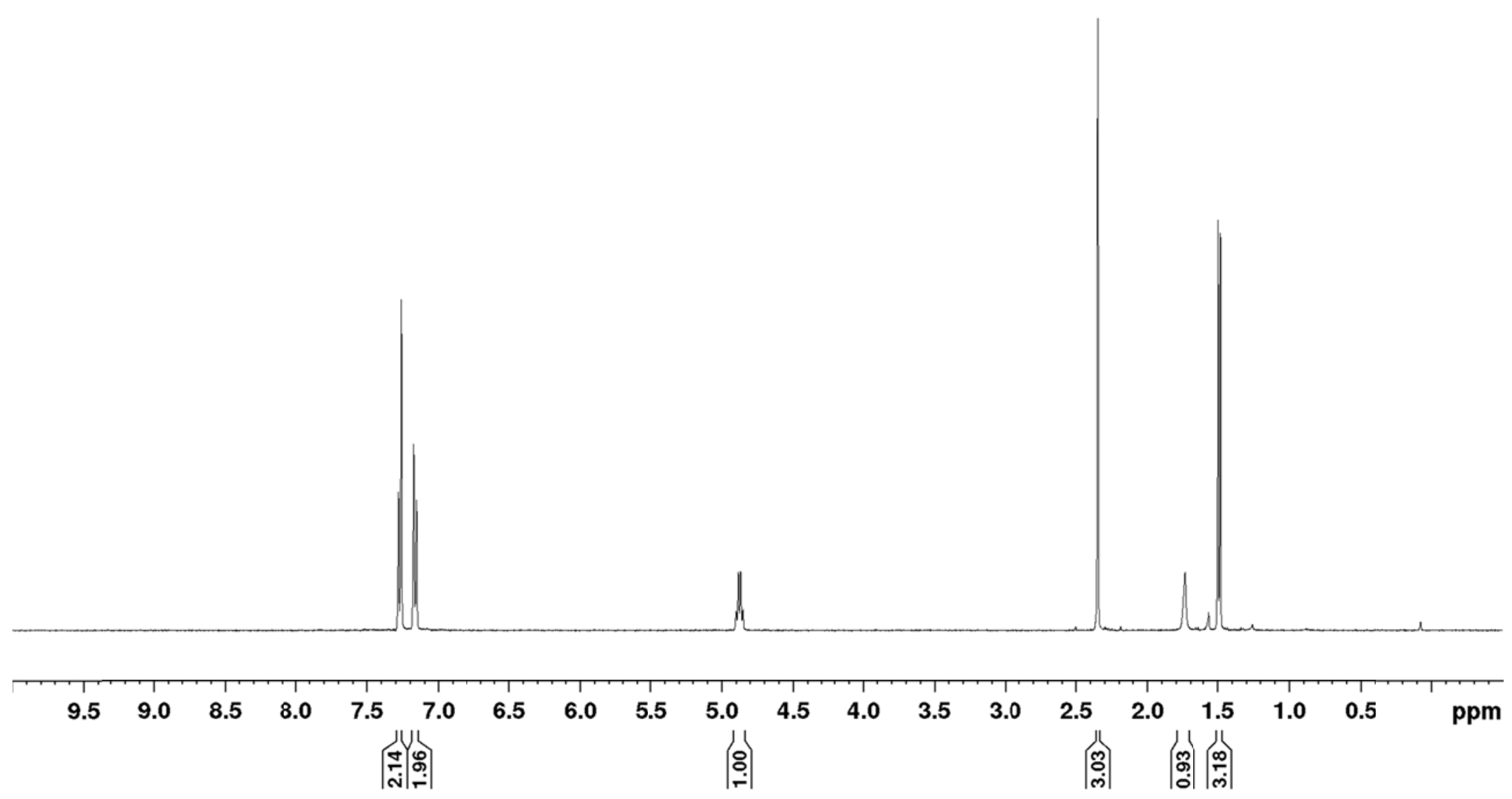


${ }^{13} \mathrm{C}\left\{{ }^{1} \mathrm{H}\right\}$ NMR $\left(101 \mathrm{MHz}, \mathrm{CDCl}_{3}\right):$
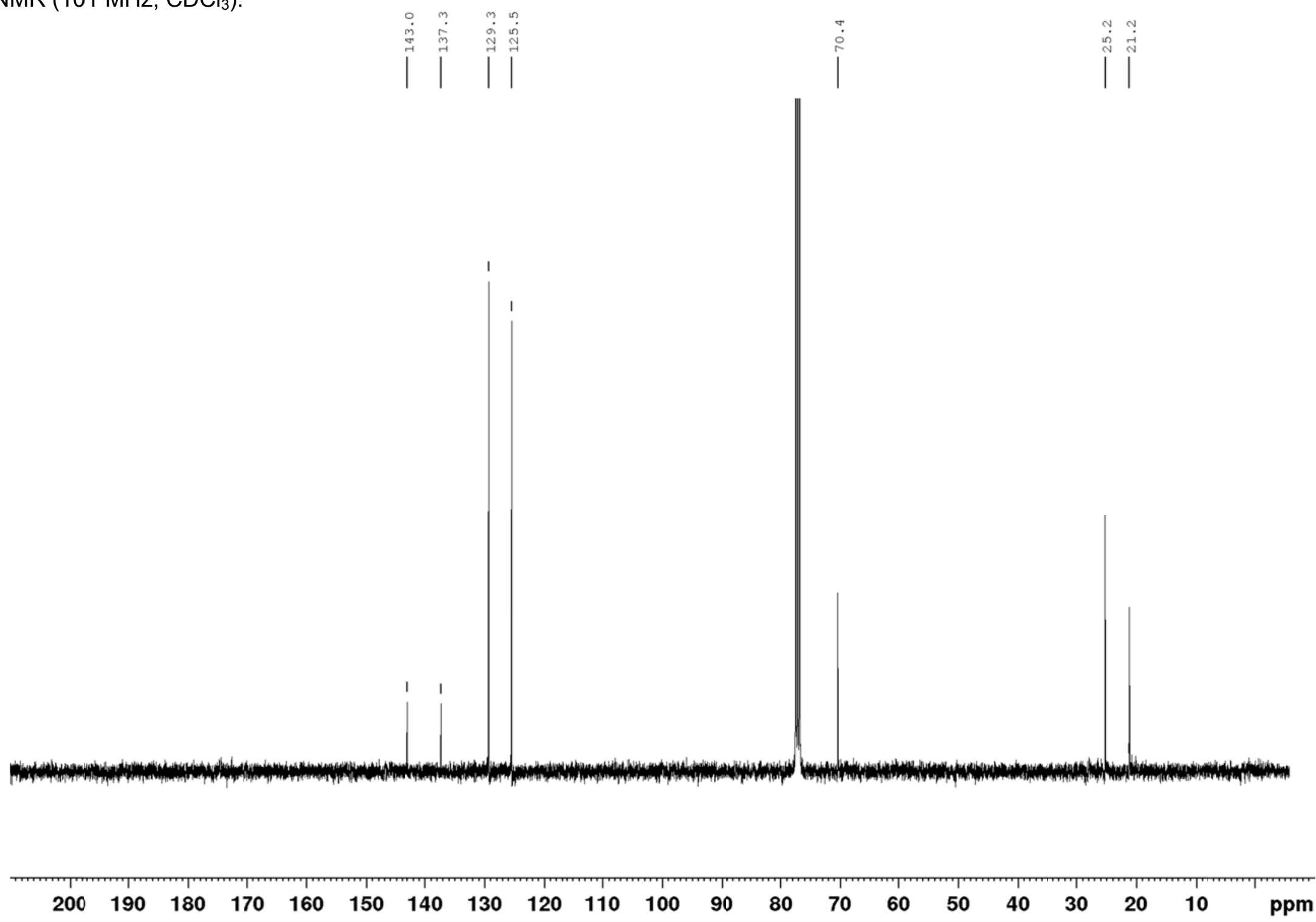


\section{(S)-1-(4-Biphenylyl)ethanol [(S)-28]}

${ }^{1} \mathrm{H}$ NMR (400 MHz, $\mathrm{CDCl}_{3}$ ):<smiles>CC(O)c1ccc(-c2ccccc2)cc1</smiles>

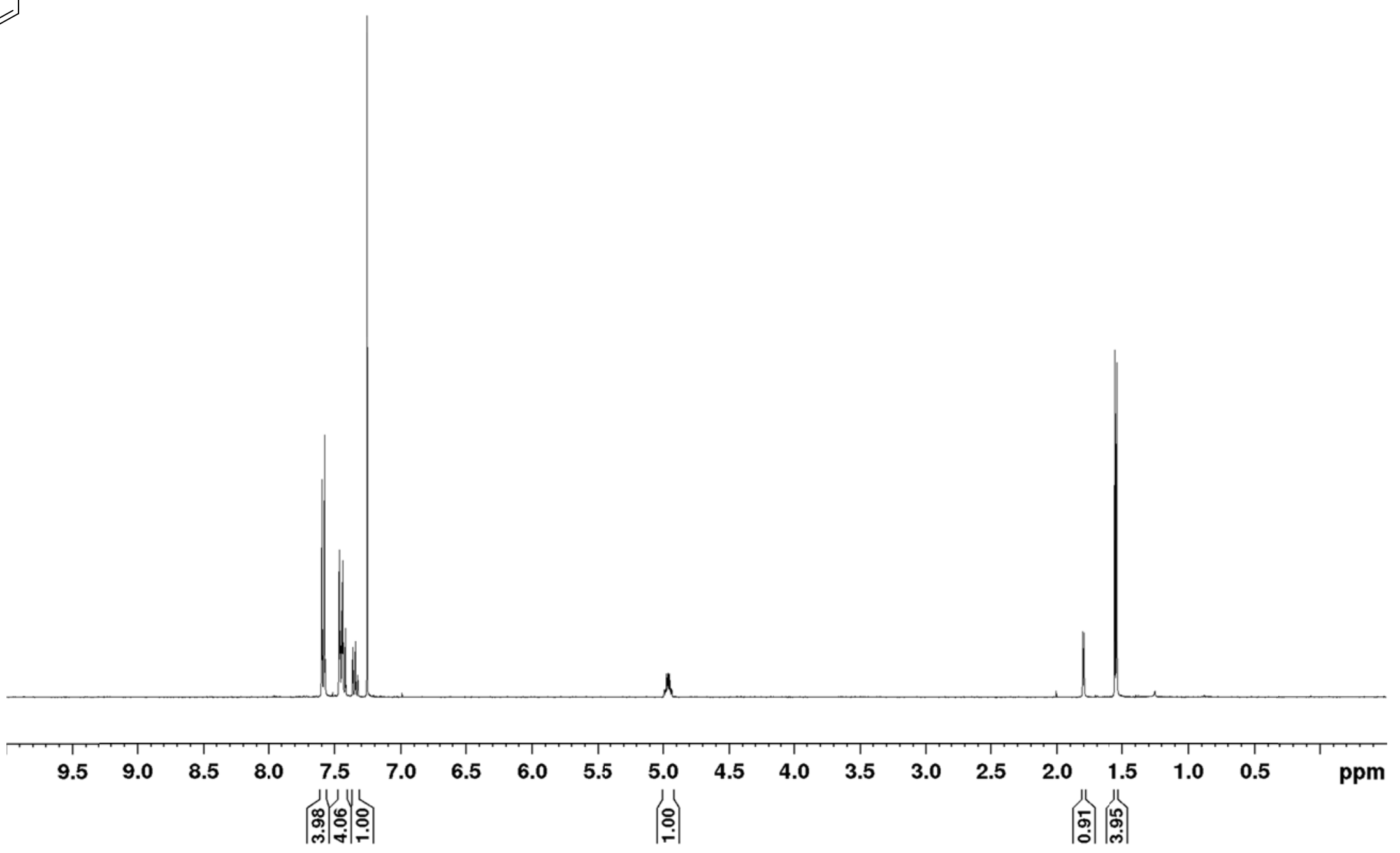


${ }^{13} \mathrm{C}\left\{{ }^{1} \mathrm{H}\right\} \operatorname{NMR}\left(101 \mathrm{MHz}, \mathrm{CDCl}_{3}\right):$

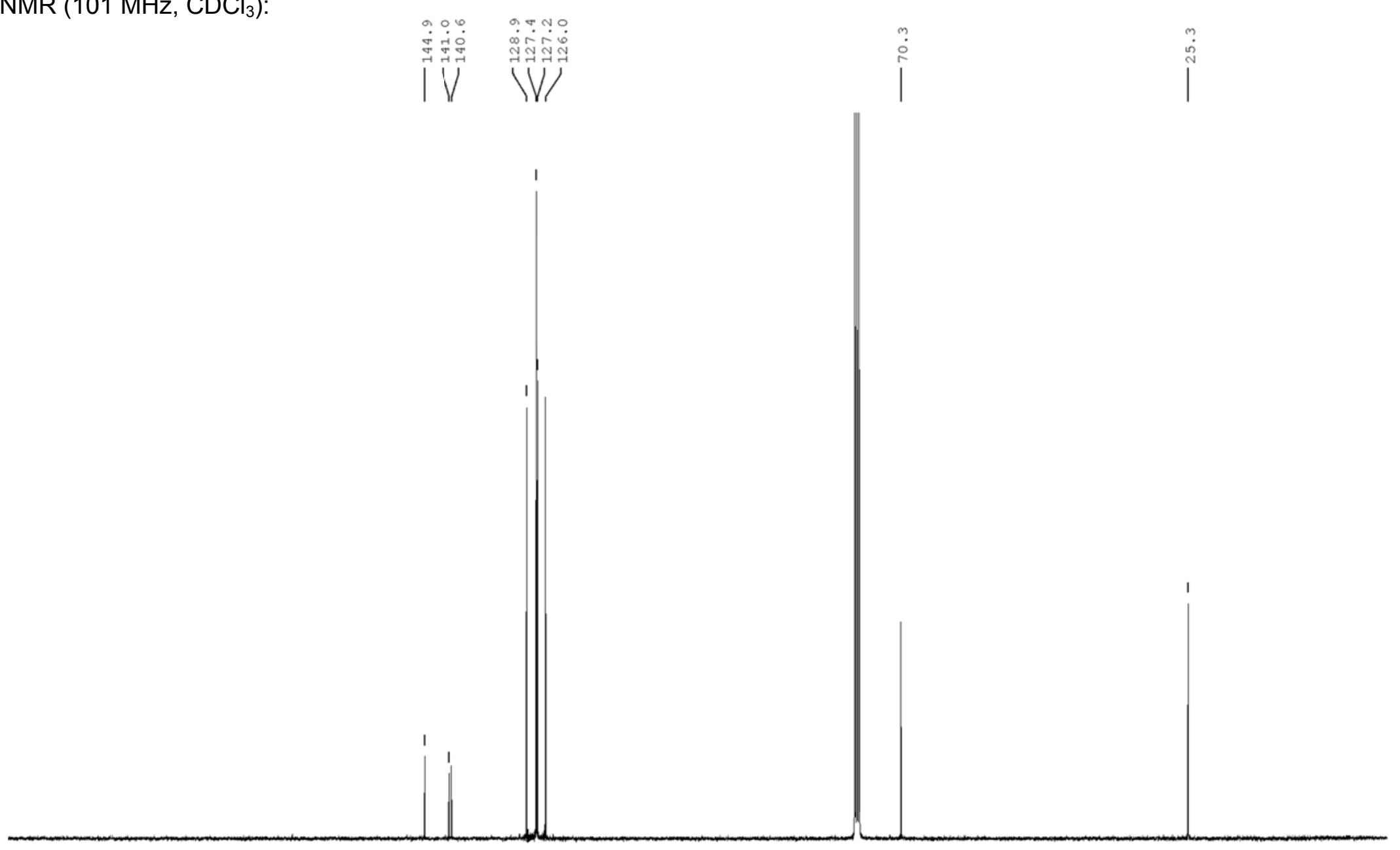

$\begin{array}{lllllllllllllllllllll}200 & 190 & 180 & 170 & 160 & 150 & 140 & 130 & 120 & 110 & 100 & 90 & 80 & 70 & 60 & 50 & 40 & 30 & 20 & 10 & \mathrm{ppm}\end{array}$ 


\section{(S)-1-(4'-Methoxyphenyl)ethanol [(S)-29]}

${ }^{1} \mathrm{H}$ NMR (400 MHz, $\left.\mathrm{CDCl}_{3}\right)$ :<smiles>COc1ccc(C(C)O)cc1</smiles>

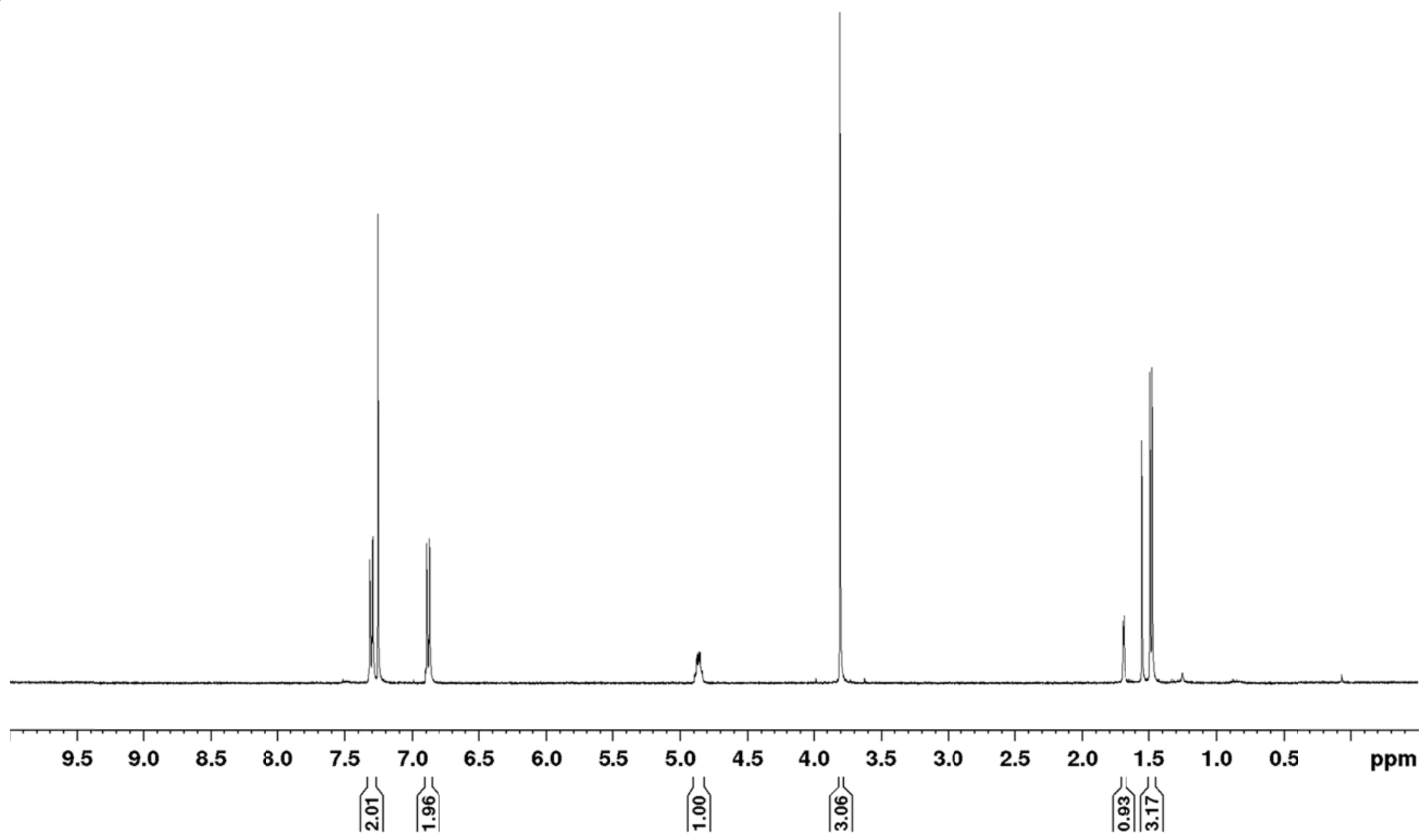


${ }^{13} \mathrm{C}\left\{{ }^{1} \mathrm{H}\right\} \operatorname{NMR}\left(101 \mathrm{MHz}, \mathrm{CDCl}_{3}\right):$

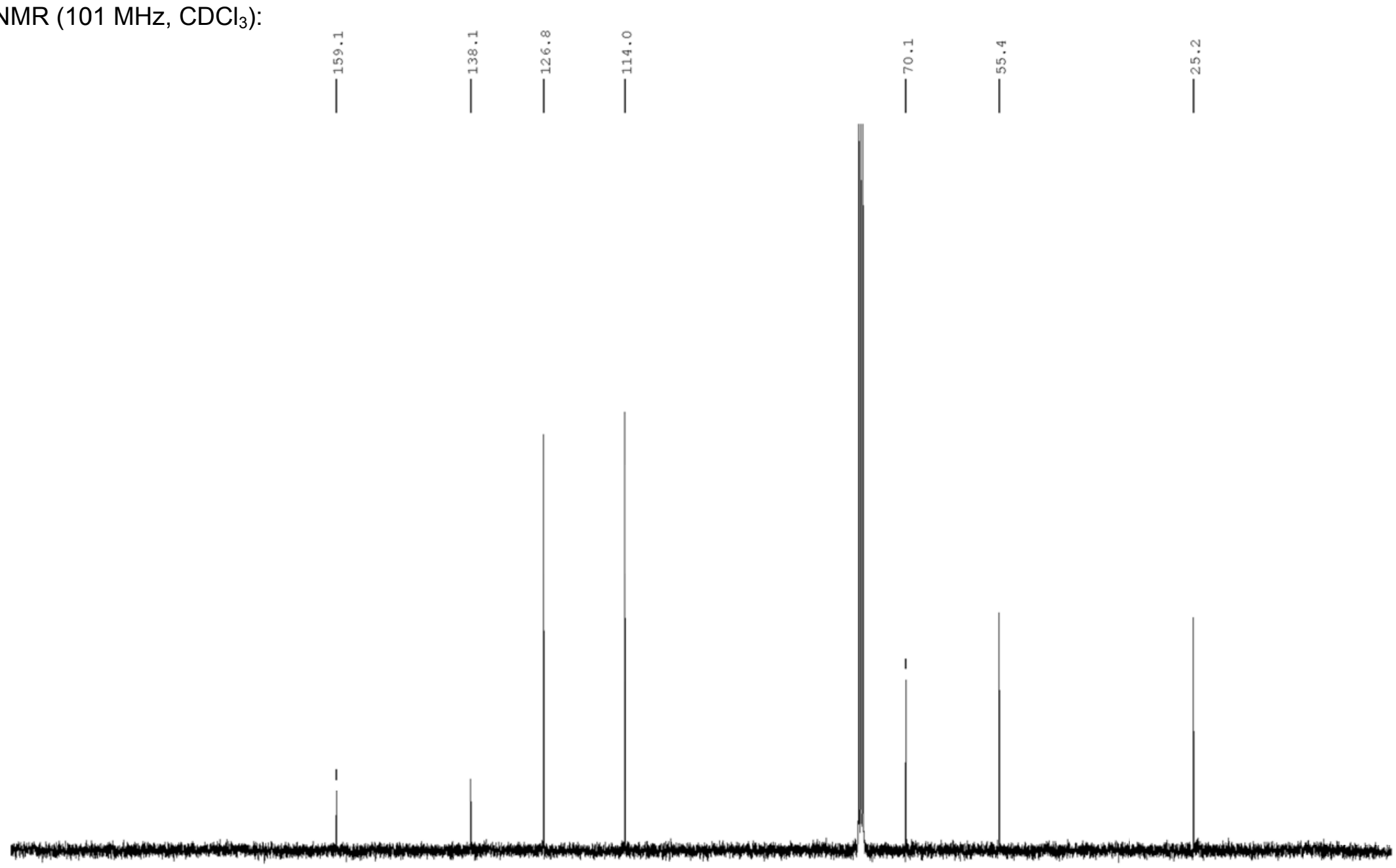

$\begin{array}{lllllllllllllllllllll}200 & 190 & 180 & 170 & 160 & 150 & 140 & 130 & 120 & 110 & 100 & 90 & 80 & 70 & 60 & 50 & 40 & 30 & 20 & 10 & \mathrm{ppm}\end{array}$ 


\section{(S)-1-(2',4',6'-Trimethylphenyl)ethanol [(S)-30]}

${ }^{1} \mathrm{H}$ NMR $\left(400 \mathrm{MHz}, \mathrm{CDCl}_{3}\right)$ :

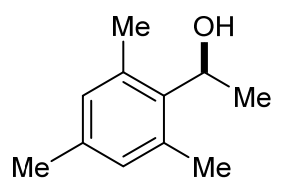$$
\mathrm{Me}
$$

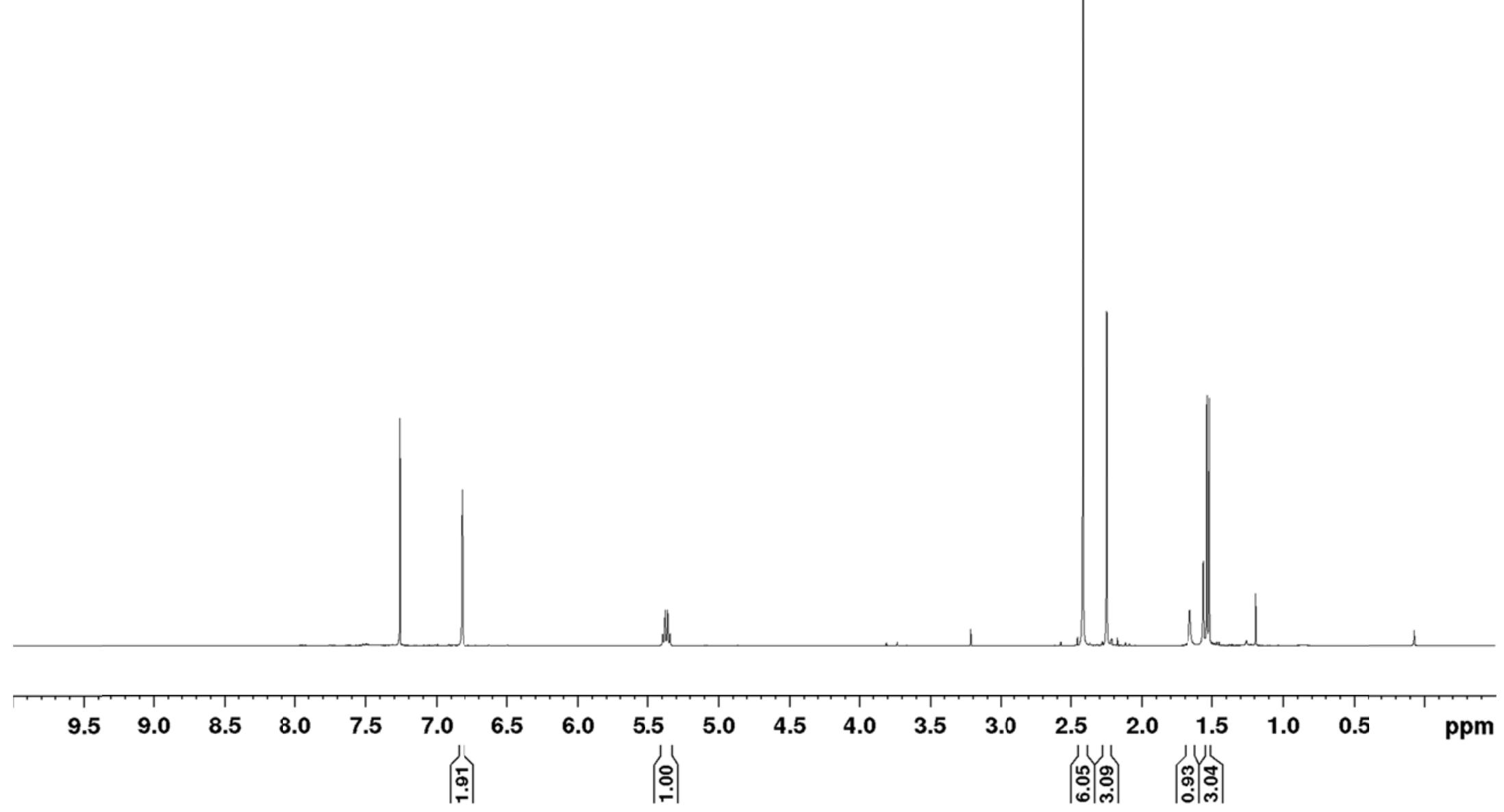


${ }^{13} \mathrm{C}\left\{{ }^{1} \mathrm{H}\right\} \operatorname{NMR}\left(101 \mathrm{MHz}, \mathrm{CDCl}_{3}\right):$
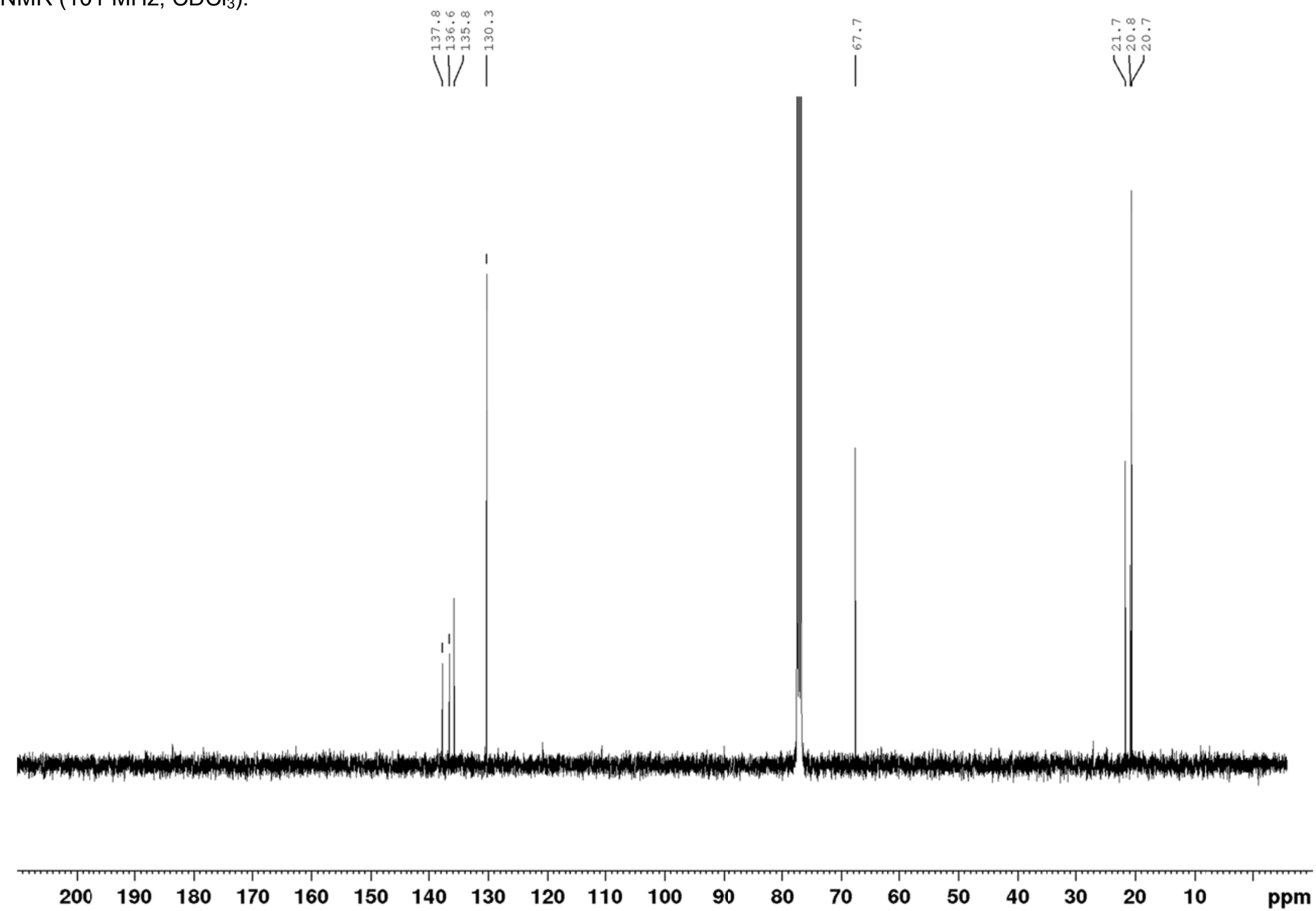


\section{(S)-1-(4'-Bromophenyl)ethanol [(S)-31]}

${ }^{1} \mathrm{H}$ NMR (400 MHz, $\left.\mathrm{C}_{6} \mathrm{D}_{6}\right)$ :<smiles>C[C@H](O)c1ccc(Br)cc1</smiles>

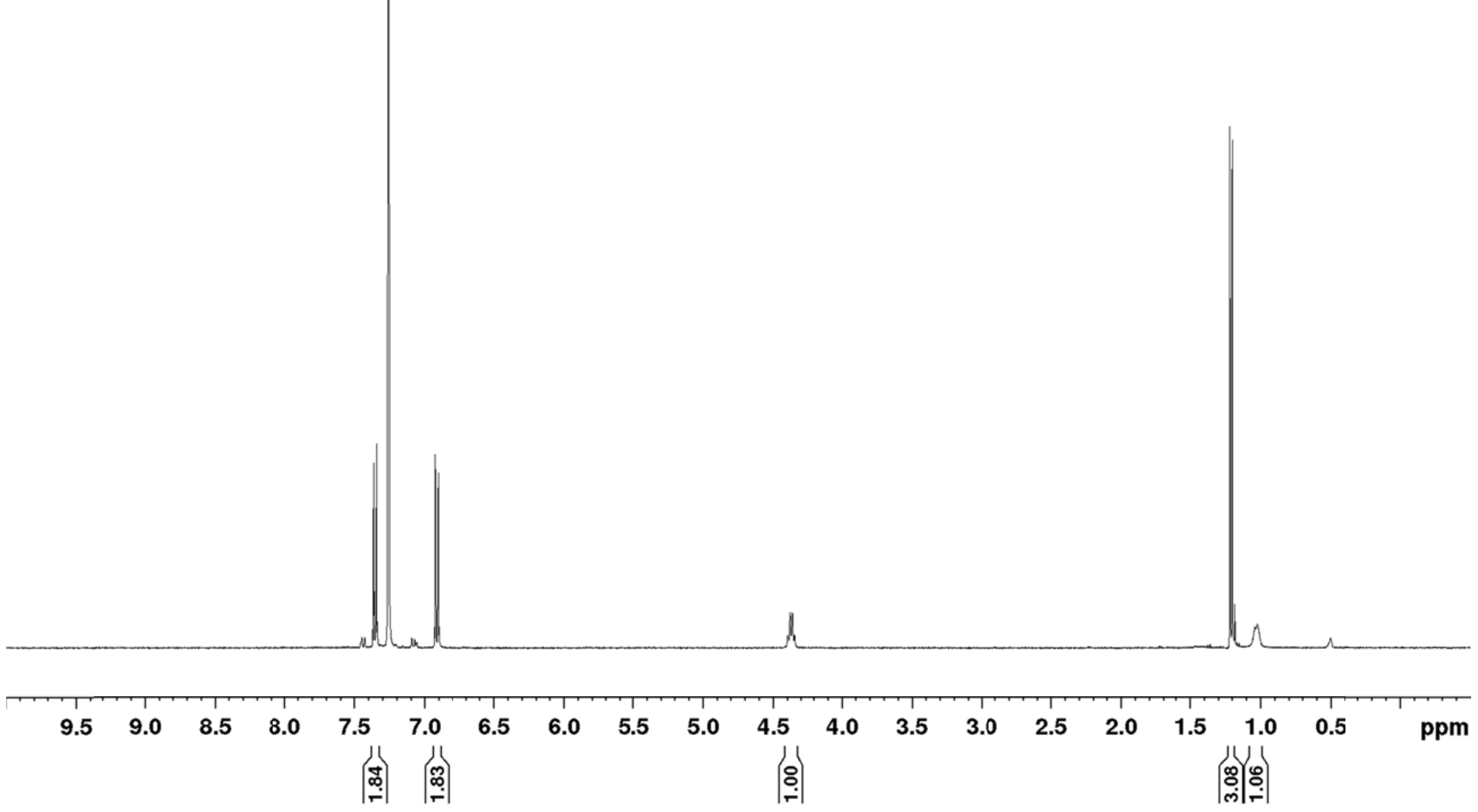


${ }^{13} \mathrm{C}\left\{{ }^{1} \mathrm{H}\right\} \operatorname{NMR}\left(101 \mathrm{MHz}, \mathrm{C}_{6} \mathrm{D}_{6}\right)$ :

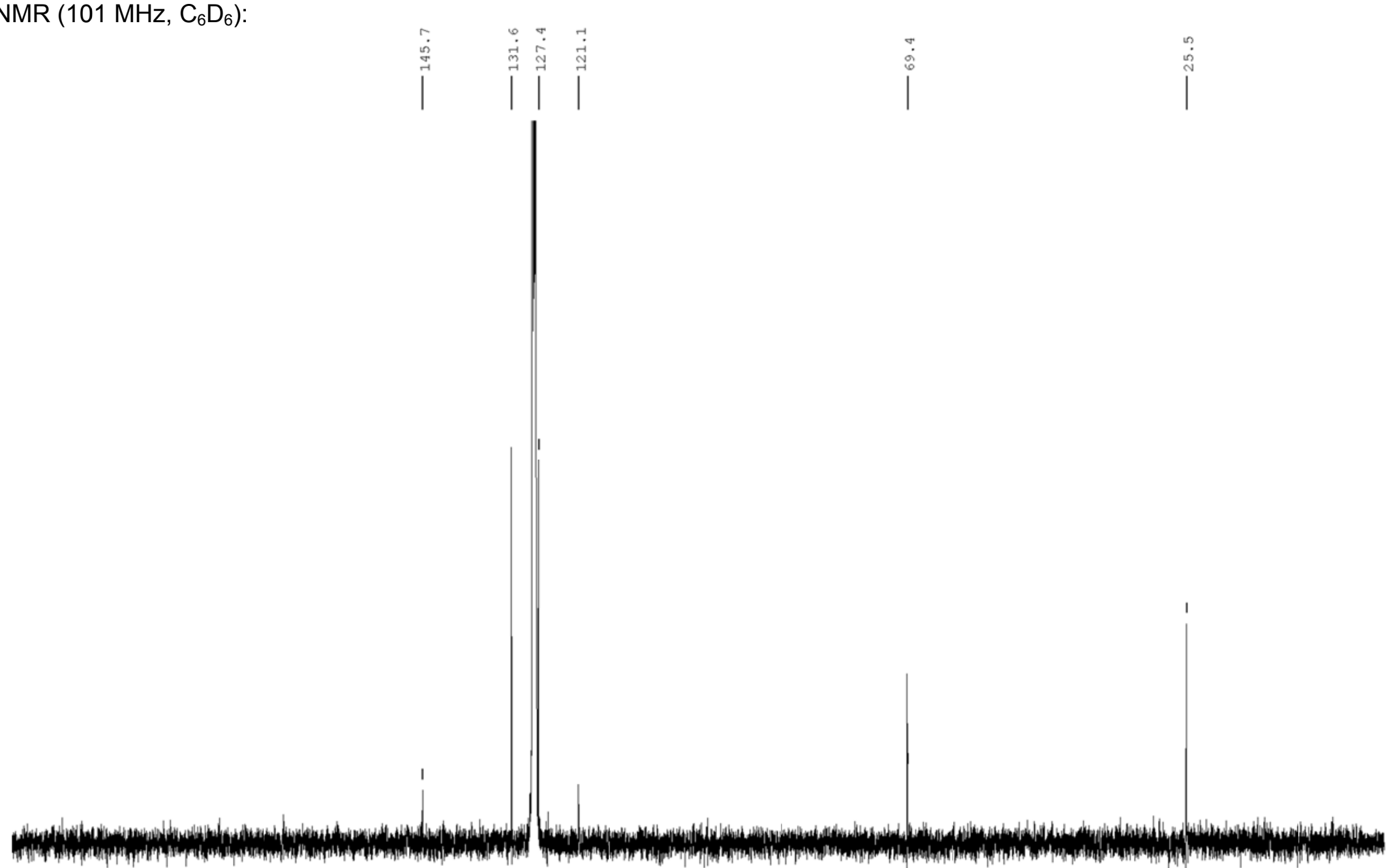

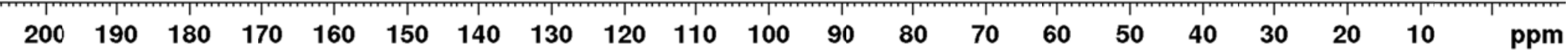




\section{(S)-1-(3'-Bromophenyl)ethanol [(S)-32]}

${ }^{1} \mathrm{H}$ NMR (400 MHz, $\left.\mathrm{C}_{6} \mathrm{D}_{6}\right)$ :<smiles>C[C@H](O)c1cccc(Br)c1</smiles>

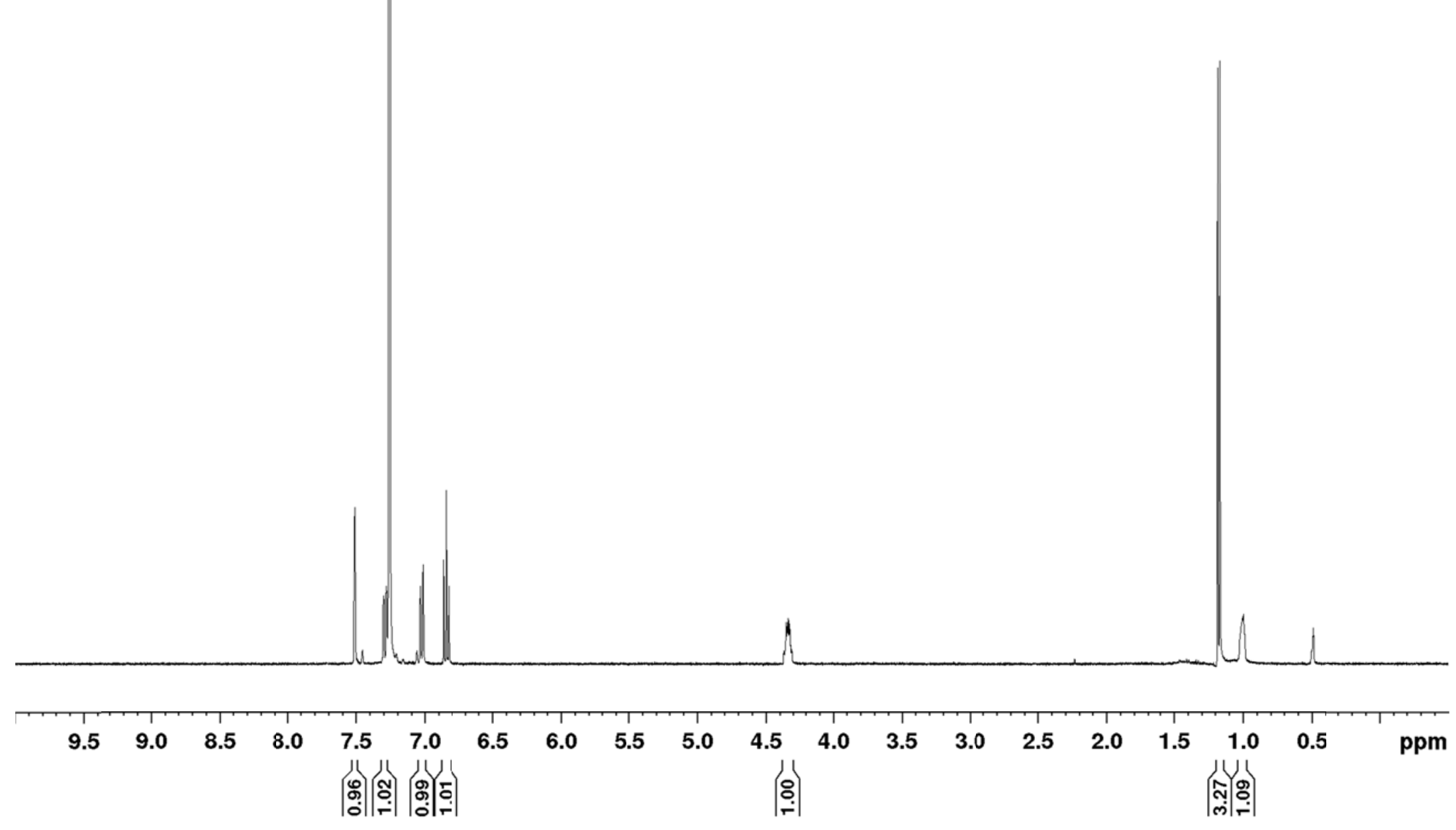


${ }^{13} \mathrm{C}\left\{{ }^{1} \mathrm{H}\right\} \operatorname{NMR}\left(101 \mathrm{MHz}, \mathrm{C}_{6} \mathrm{D}_{6}\right):$

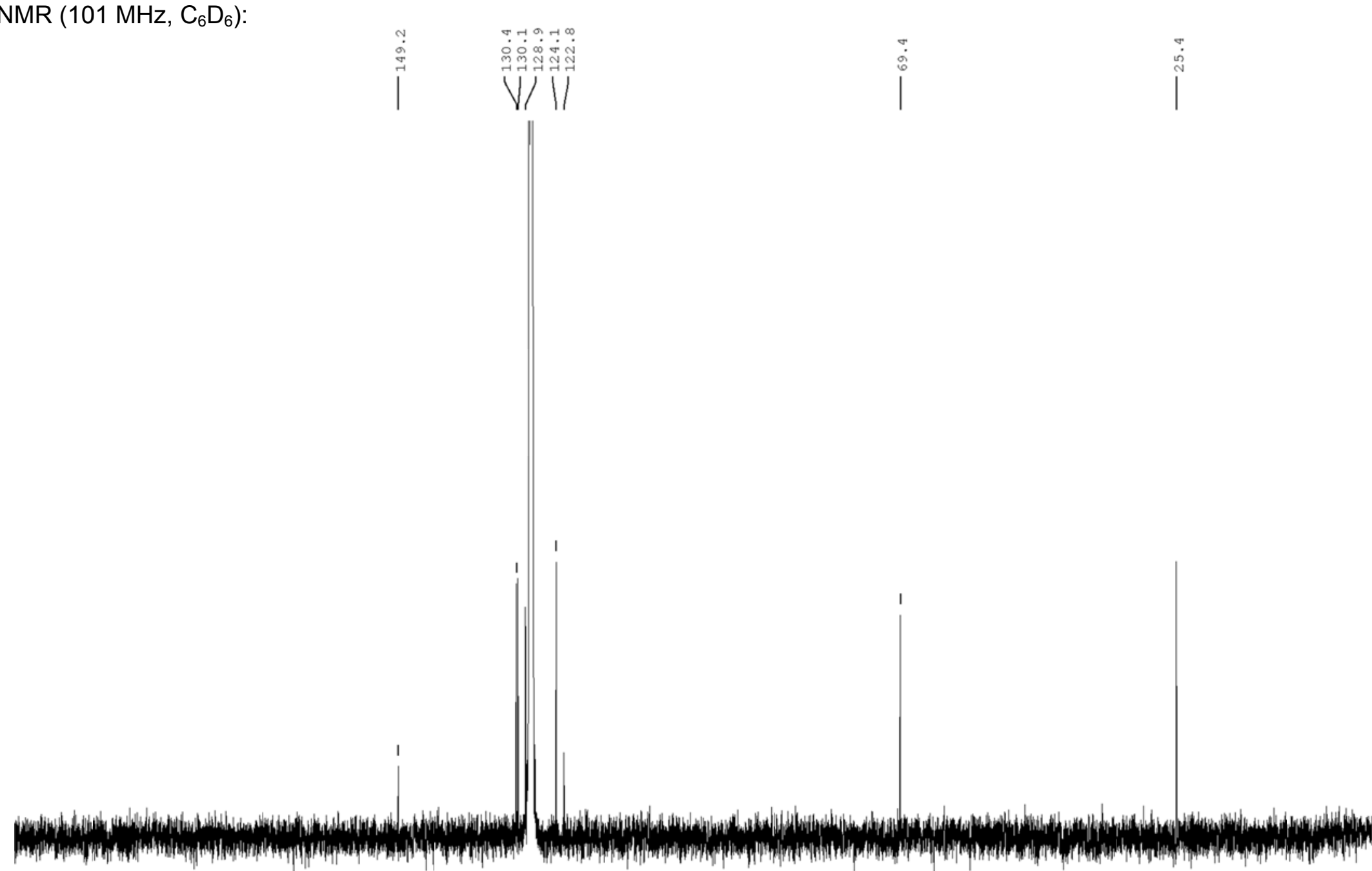

$\begin{array}{lllllllllll}200 & 190 & 180 & 170 & 160 & 150 & 140 & 130 & 120 & 110 & 100\end{array}$

80

$70 \quad 60$

$\begin{array}{lllllll}50 & 40 & 30 & 20 & 10 & \text { ppm }\end{array}$




\section{(S)-1-(2'-Bromophenyl)ethanol [(S)-33]}

${ }^{1} \mathrm{H}$ NMR (500 MHz, $\mathrm{CDCl}_{3}$ ):<smiles>CC(O)c1ccccc1Br</smiles>

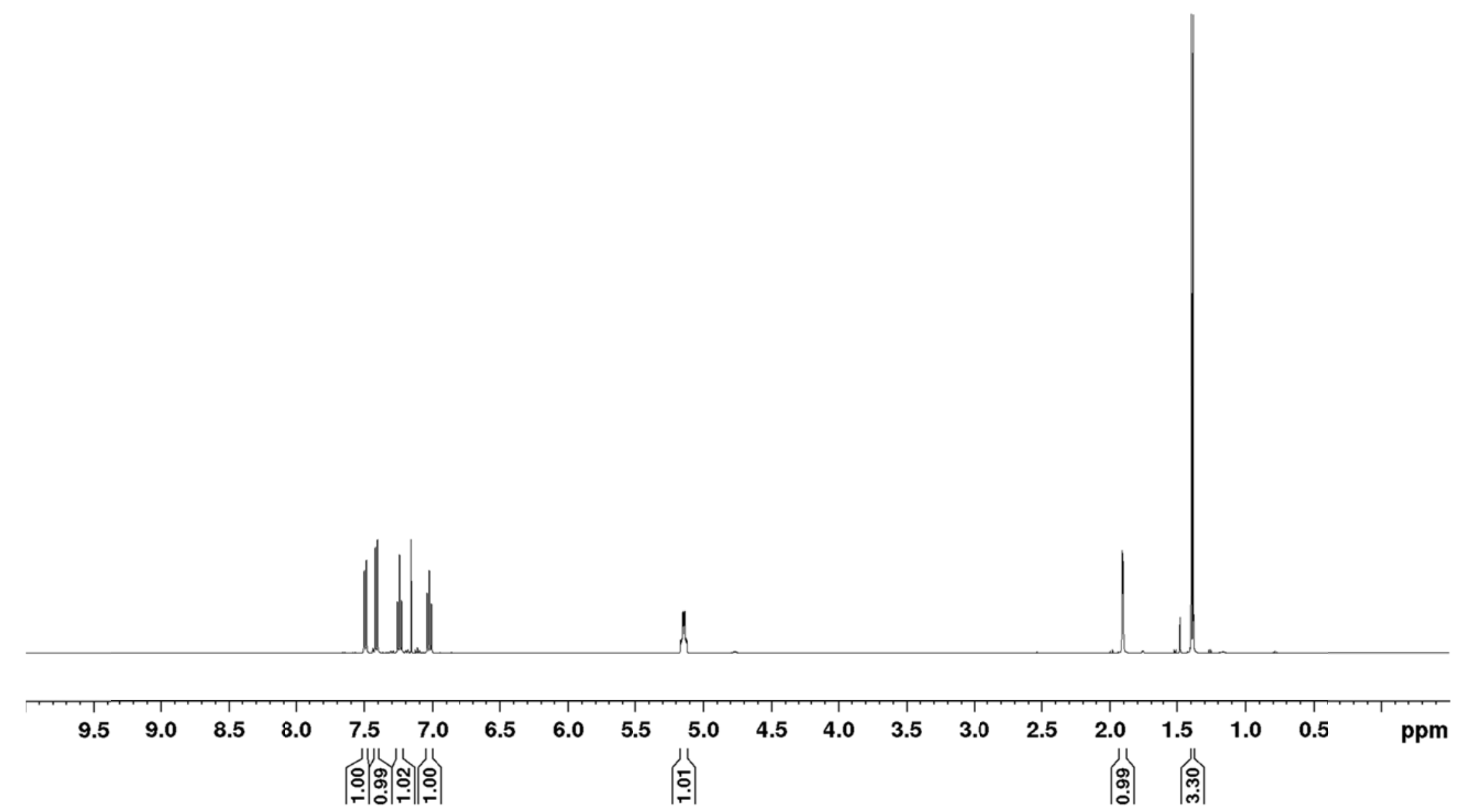


$\left.{ }^{13} \mathrm{C}\left\{{ }^{1} \mathrm{H}\right\} \operatorname{NMR}(126 \mathrm{MHz}, \mathrm{CDCl})_{3}\right)$

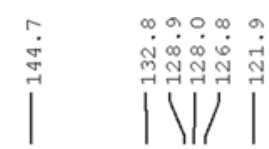

$\stackrel{\square}{\circ}$

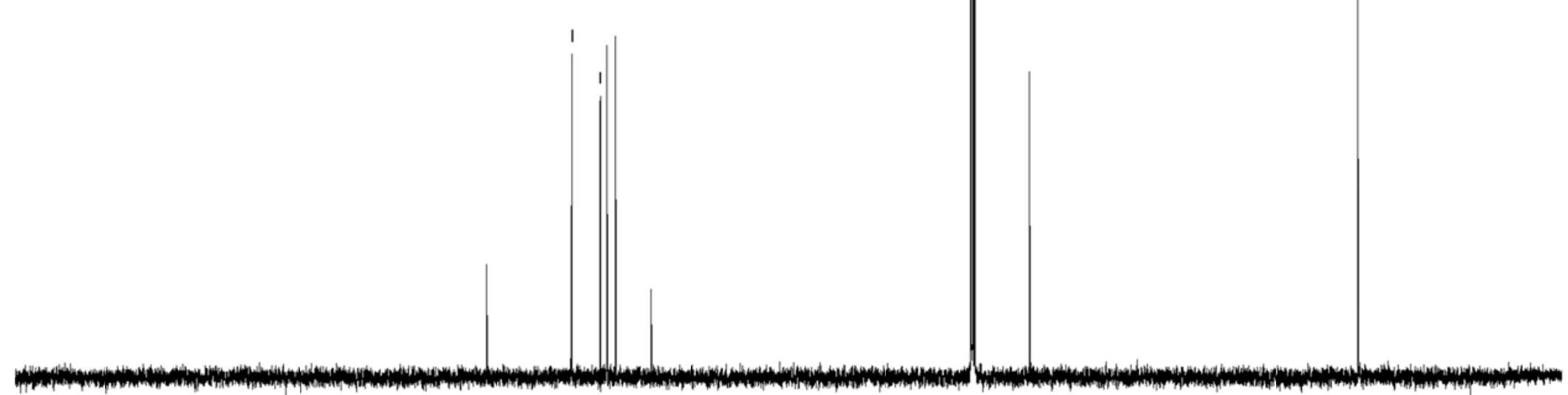

$\begin{array}{lllllllllllllllllllll}200 & 190 & 180 & 170 & 160 & 150 & 140 & 130 & 120 & 110 & 100 & 90 & 80 & 70 & 60 & 50 & 40 & 30 & 20 & 10 & \mathrm{ppm}\end{array}$ 


\section{(S)-1,2-Diphenylethanol [(S)-34]}

${ }^{1} \mathrm{H}$ NMR $\left(400 \mathrm{MHz}, \mathrm{CDCl}_{3}\right)$ :
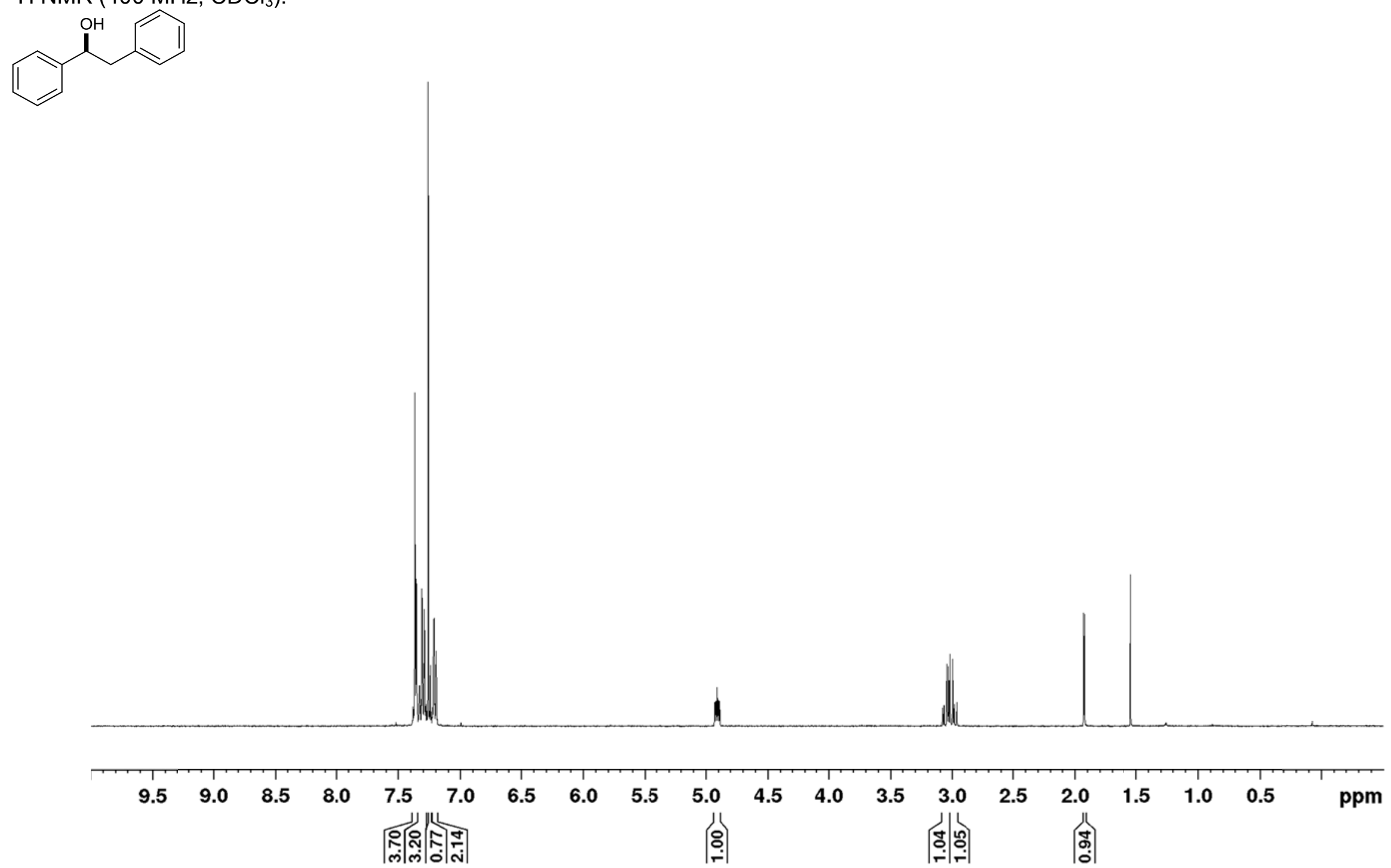
${ }^{13} \mathrm{C}\left\{{ }^{1} \mathrm{H}\right\} \operatorname{NMR}\left(101 \mathrm{MHz}, \mathrm{CDCl}_{3}\right)$ :
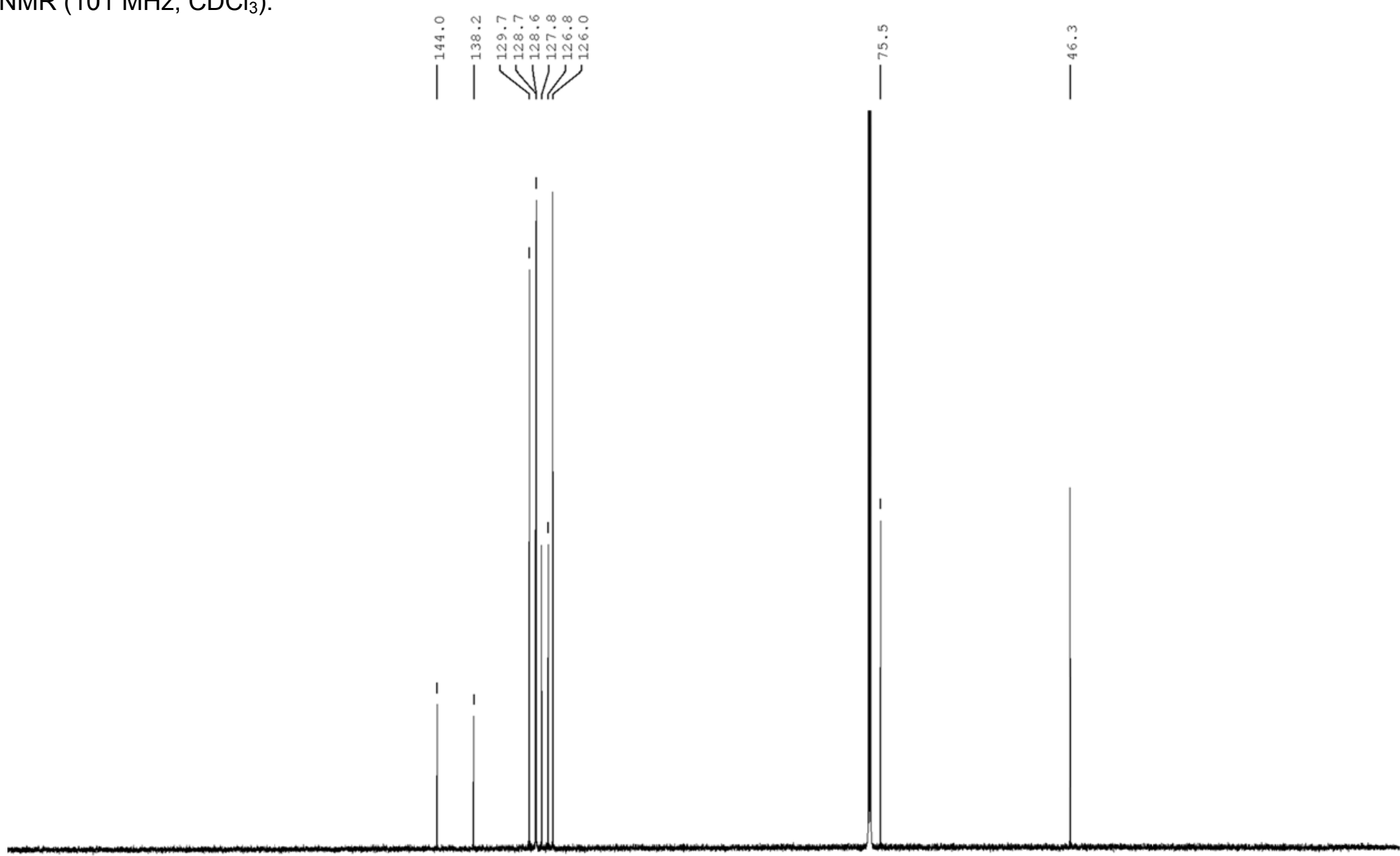

$\begin{array}{llllllllllllllllllllllll}200 & 190 & 180 & 170 & 160 & 150 & 140 & 130 & 120 & 110 & 100 & 90 & 80 & 70 & 60 & 50 & 40 & 30 & 20 & 10 & \text { ppm }\end{array}$ 
Supporting Information for the Journal of the American Chemical Society

S67

(S)-Cyclohexyl(phenyl)methanol [(S)-35]

${ }^{1} \mathrm{H}$ NMR (400 MHz, $\left.\mathrm{CDCl}_{3}\right)$ :<smiles>OC(c1ccccc1)C1CCCCC1</smiles>

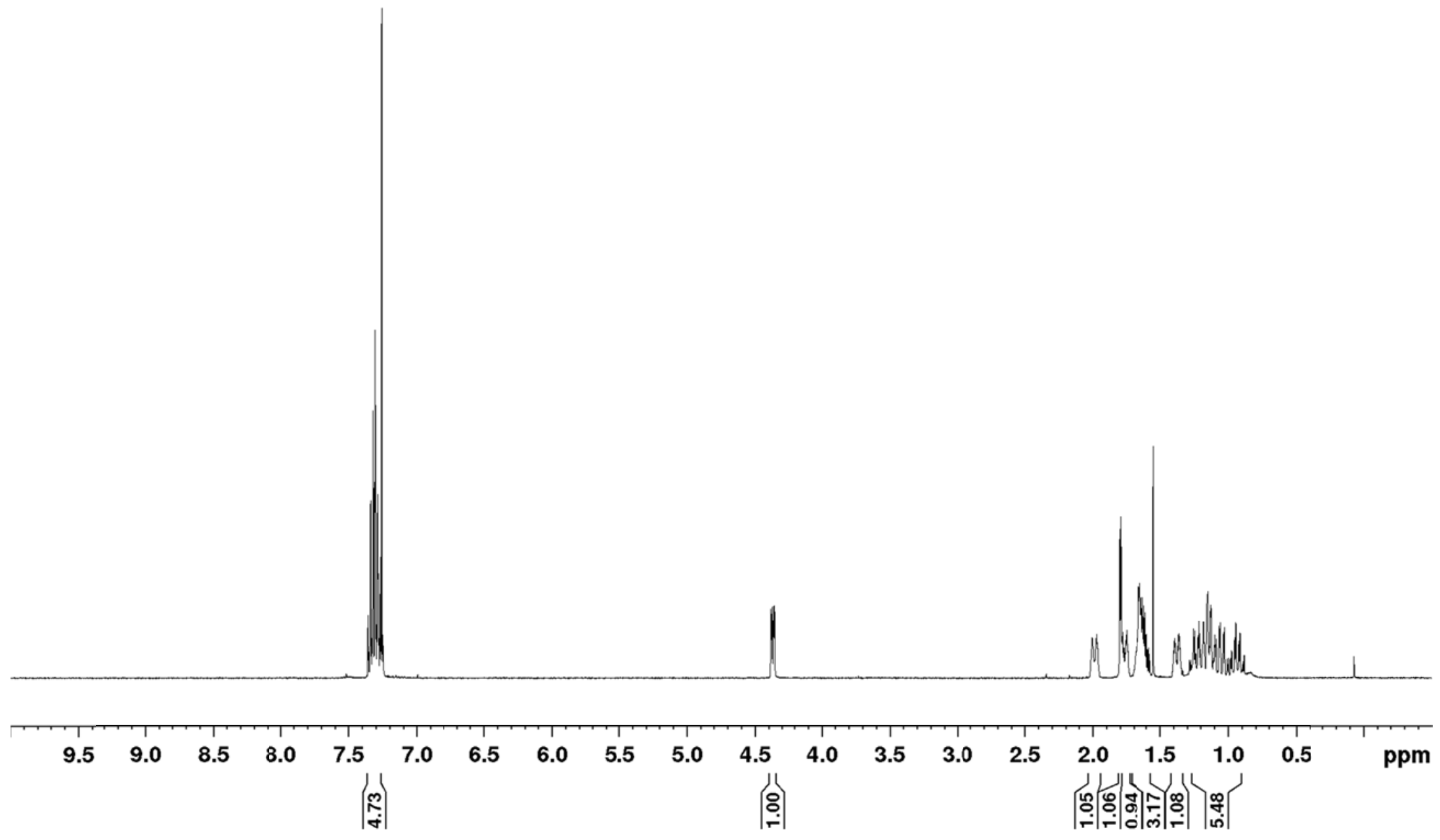


${ }^{13} \mathrm{C}\left\{{ }^{1} \mathrm{H}\right\}$ NMR $\left(101 \mathrm{MHz}, \mathrm{CDCl}_{3}\right):$

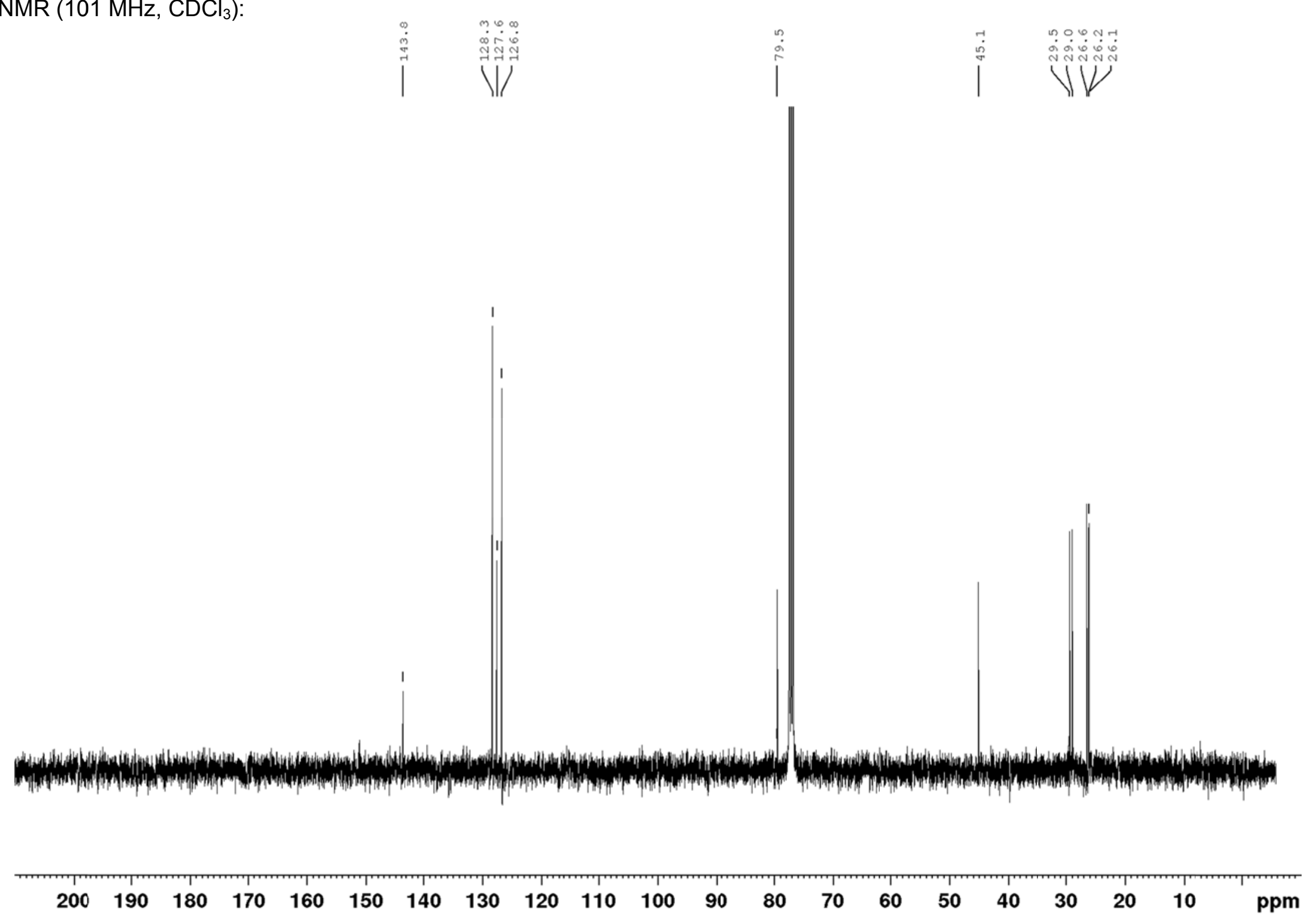




\section{(S)-1-(2'-Naphthyl)ethanol [(S)-36]}

${ }^{1} \mathrm{H}$ NMR (400 MHz, $\mathrm{CDCl}_{3}$ ):

${ }_{M e}^{\mathrm{OH}}$
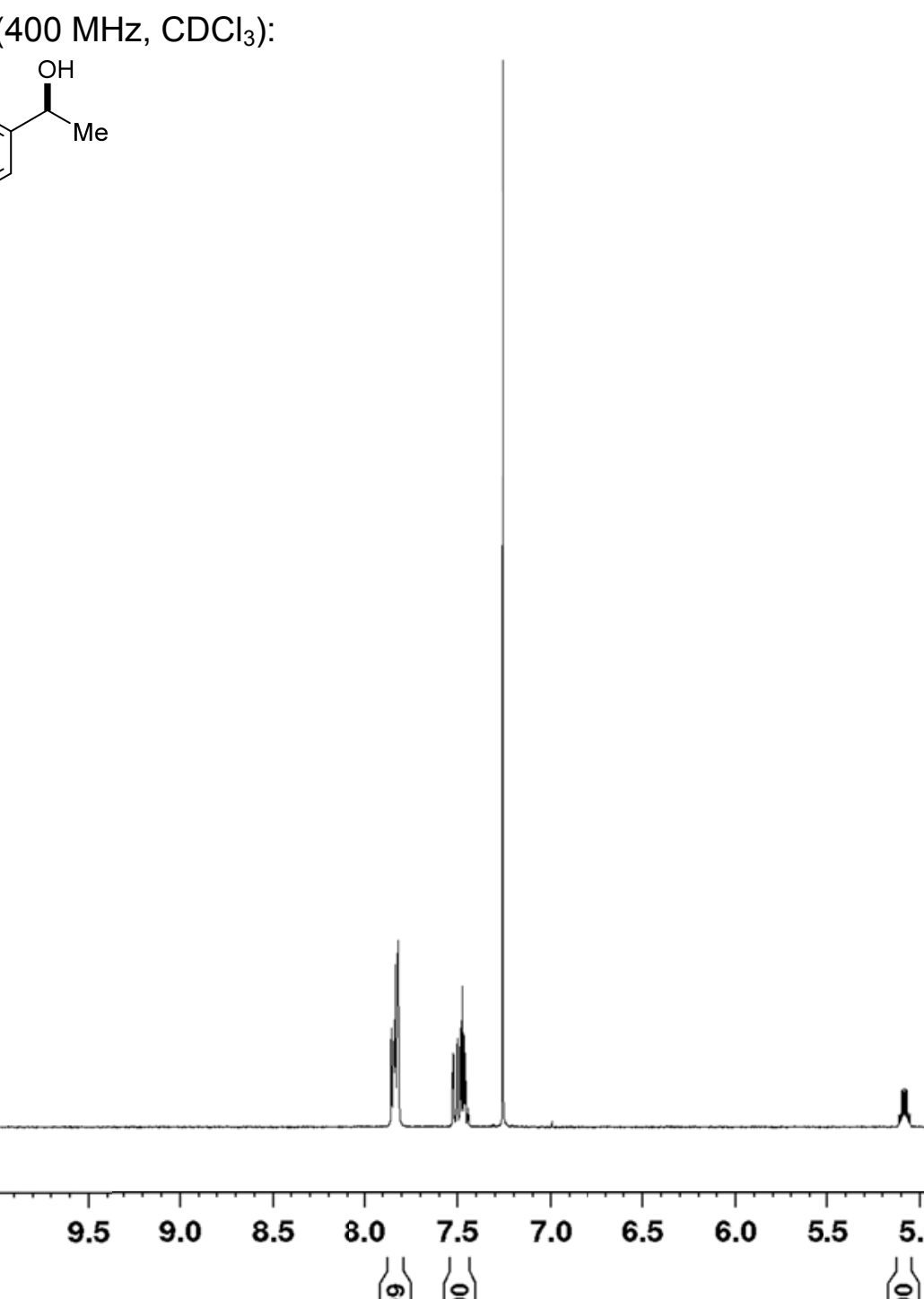

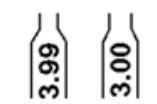

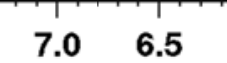

5.55

|

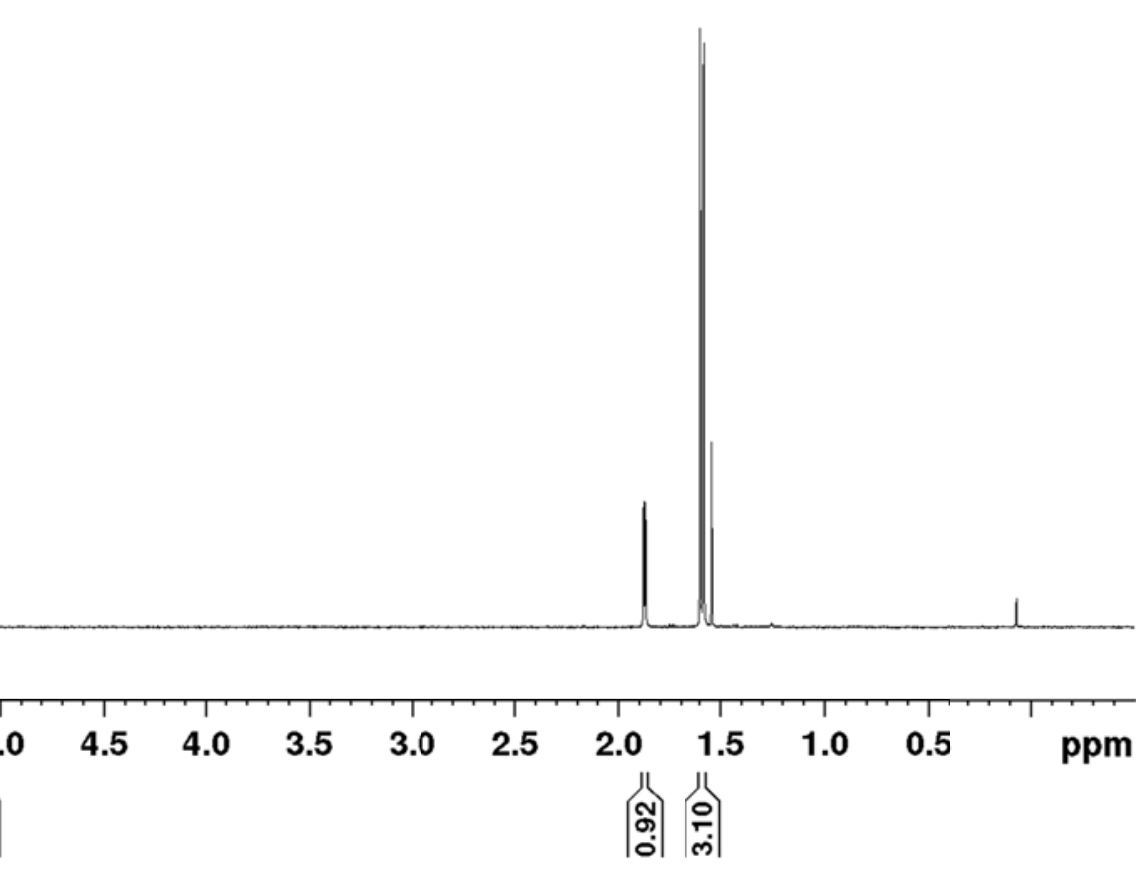


${ }^{13} \mathrm{C}\left\{{ }^{1} \mathrm{H}\right\}$ NMR $\left(101 \mathrm{MHz}, \mathrm{CDCl}_{3}\right):$

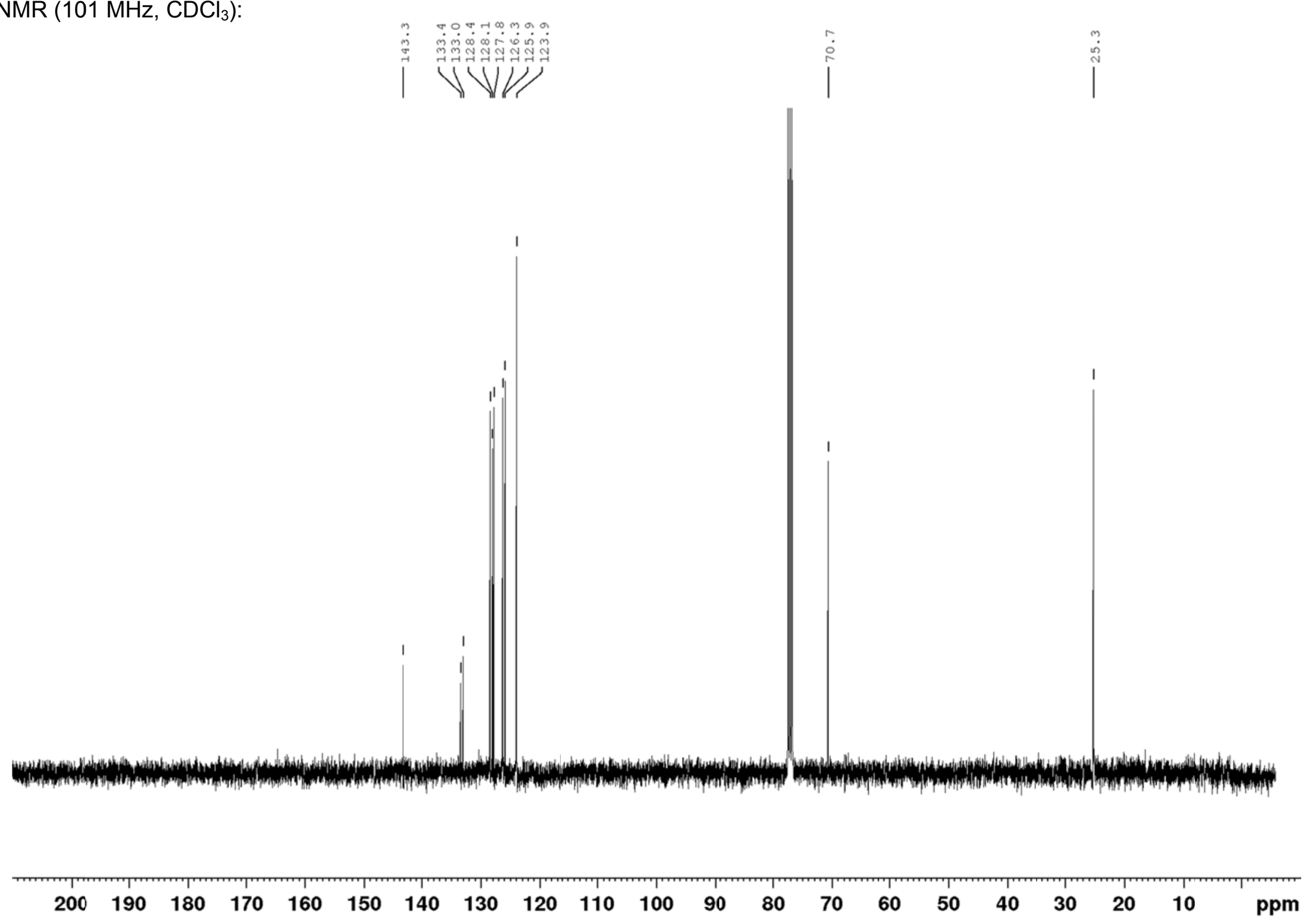




\section{(S)-4'-Bromophenyl(phenyl)methanol [(S)-37]}

${ }^{1} \mathrm{H}$ NMR (400 MHz, $\left.\mathrm{CDCl}_{3}\right)$ :<smiles>OC(c1ccccc1)c1ccc(Br)cc1</smiles>

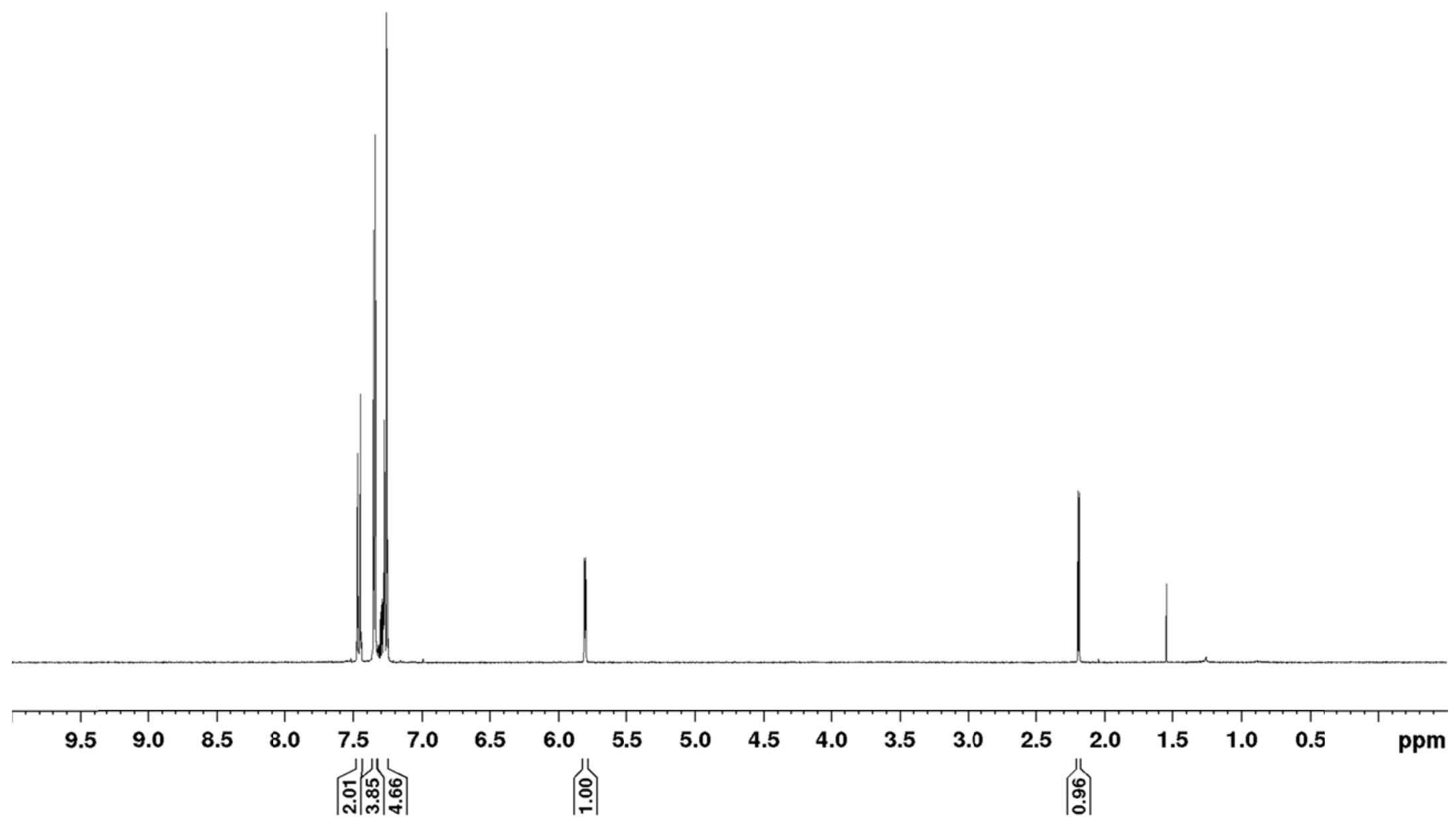


${ }^{13} \mathrm{C}\left\{{ }^{1} \mathrm{H}\right\}$ NMR $\left(101 \mathrm{MHz}, \mathrm{CDCl}_{3}\right):$
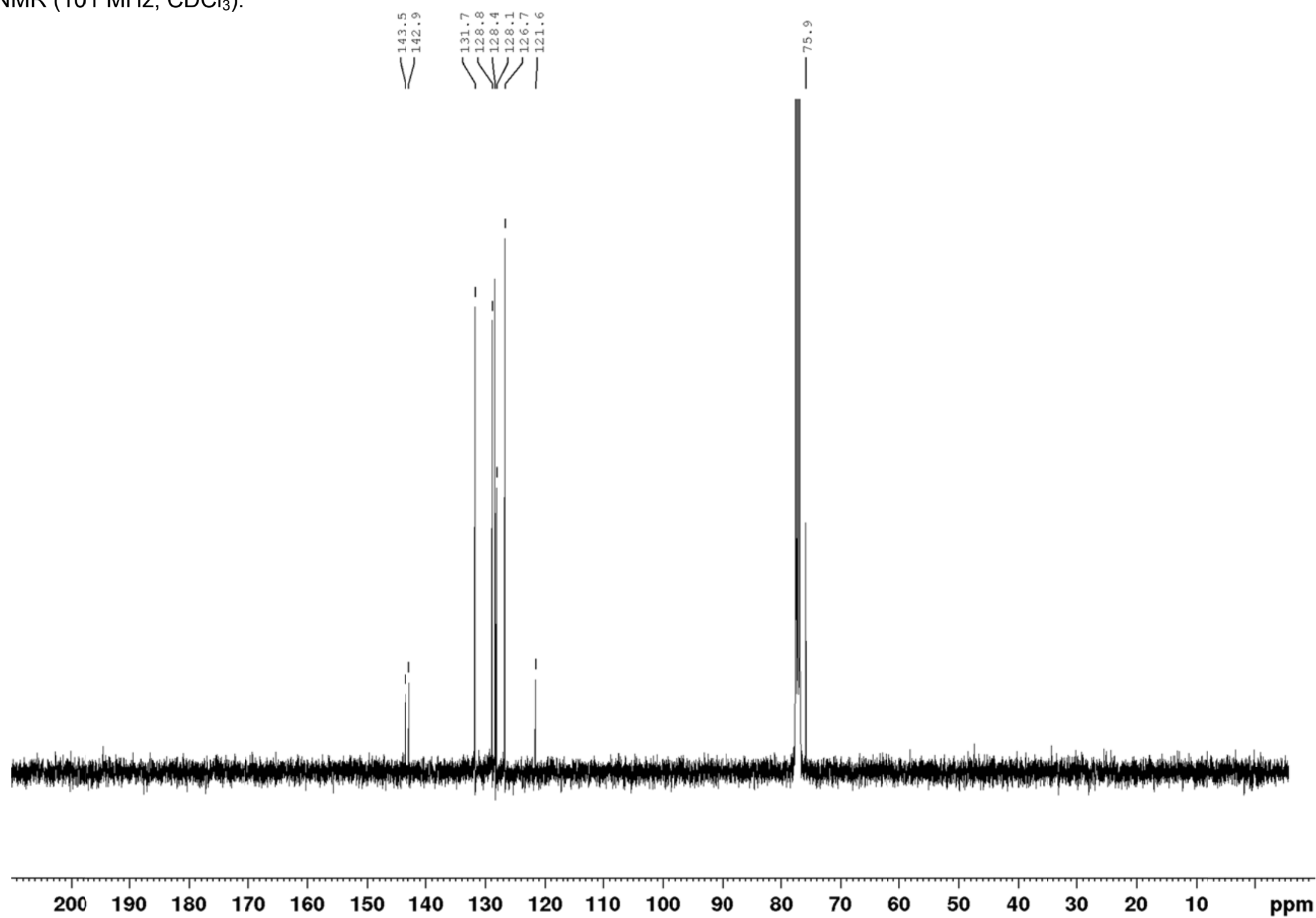


\section{HPLC Traces of Alcohols 7 and 23-37}

\section{(S)-1-Phenylethanol [(S)-7]}

The enantiomeric excess was determined by HPLC analysis on a chiral stationary phase (Daicel Chiralcel OJ-H column, column temperature $20^{\circ} \mathrm{C}$, solvent $n$-heptane:iso-propanol $=$ 98:2, flow rate $0.8 \mathrm{~mL} / \mathrm{min}, \lambda=210 \mathrm{~nm}): t_{\mathrm{R}}=25.8 \mathrm{~min}$ for $(S)-7, t_{\mathrm{R}}=30.8 \mathrm{~min}$ for $(R)-7$.

rac-1-Phenylethanol (rac-7, purchased from Sigma Aldrich)

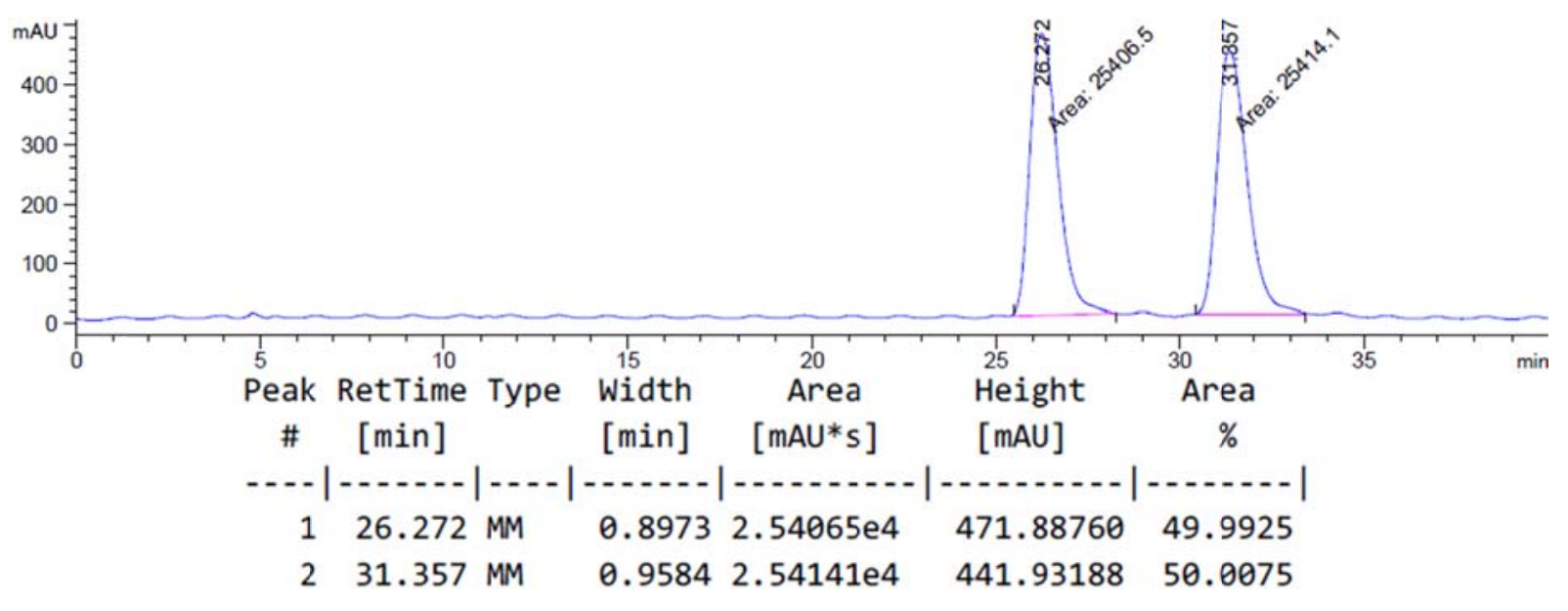

(S)-1-Phenylethanol [(S)-7, 93\% ee]

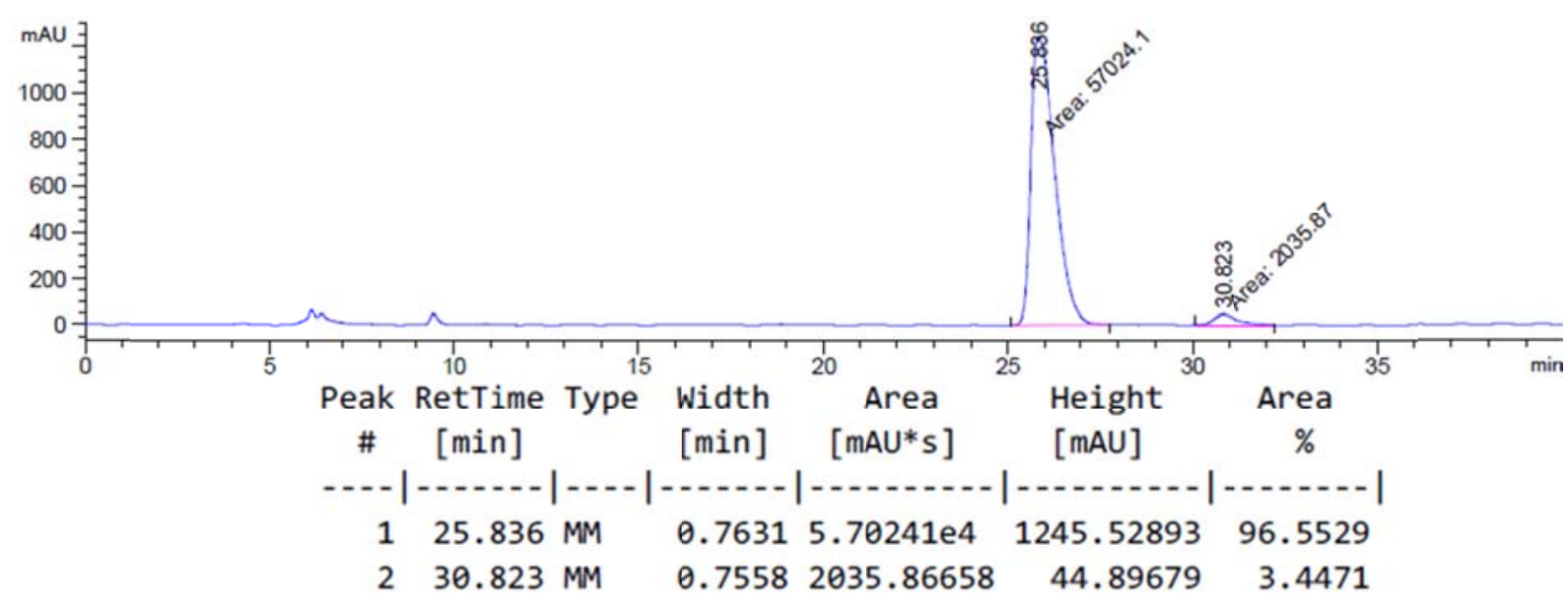




\section{(S)-1-(4'-Trifluoromethylphenyl)ethanol [(S)-23]}

The enantiomeric excess was determined by HPLC analysis on a chiral stationary phase (Daicel Chiralcel AD-H column, column temperature $20^{\circ} \mathrm{C}$, solvent $n$-heptane:iso-propanol = 99.7:0.3, flow rate $0.8 \mathrm{~mL} / \mathrm{min}, \lambda=210 \mathrm{~nm}): t_{\mathrm{R}}=71.7 \mathrm{~min}$ for $(S)-23, t_{\mathrm{R}}=77.7 \mathrm{~min}$ for $(R)-23$.

rac-1-(4'-Trifluoromethylphenyl)ethanol (rac-23, synthesized according to GP 3)

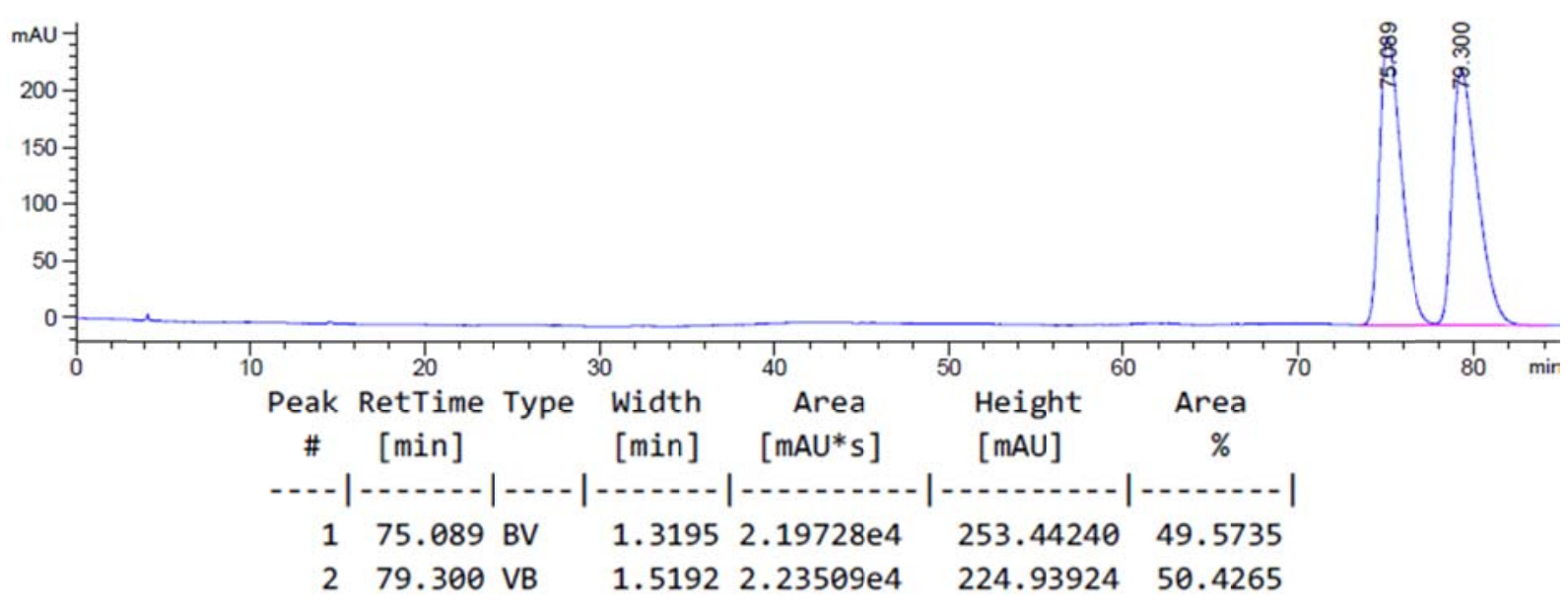

(S)-1-(4'-Trifluoromethylphenyl)ethanol [(S)-23, 82\% ee]

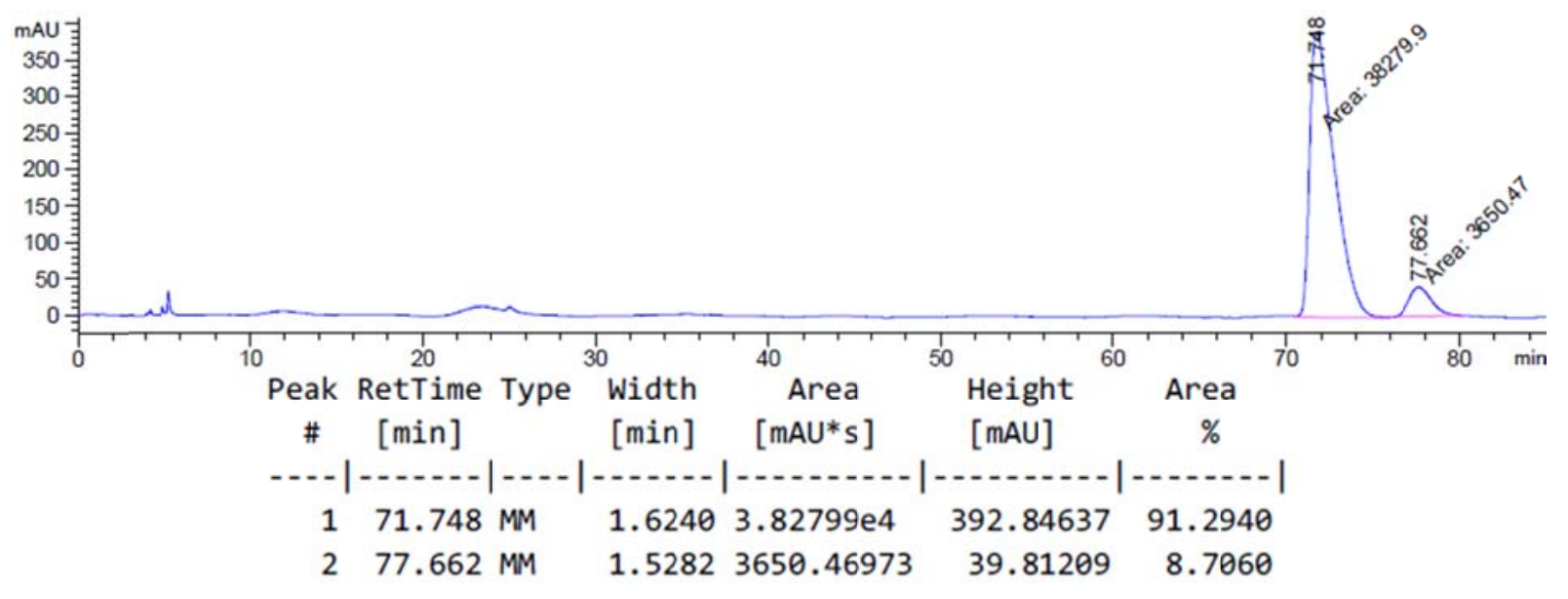




\section{(S)-1-(4'-Nitrophenyl)ethanol [(S)-24]}

The enantiomeric excess was determined by HPLC analysis on a chiral stationary phase (Daicel Chiralcel OJ-H column, column temperature $20^{\circ} \mathrm{C}$, solvent $n$-heptane:iso-propanol = 95:5, flow rate $0.8 \mathrm{~mL} / \mathrm{min}, \lambda=210 \mathrm{~nm}): t_{\mathrm{R}}=40.6 \mathrm{~min}$ for $(S)-24, t_{\mathrm{R}}=44.8 \mathrm{~min}$ for $(R)-24$.

rac-1-(4'-Nitrophenyl)ethanol (rac-24, synthesized according to GP 3)

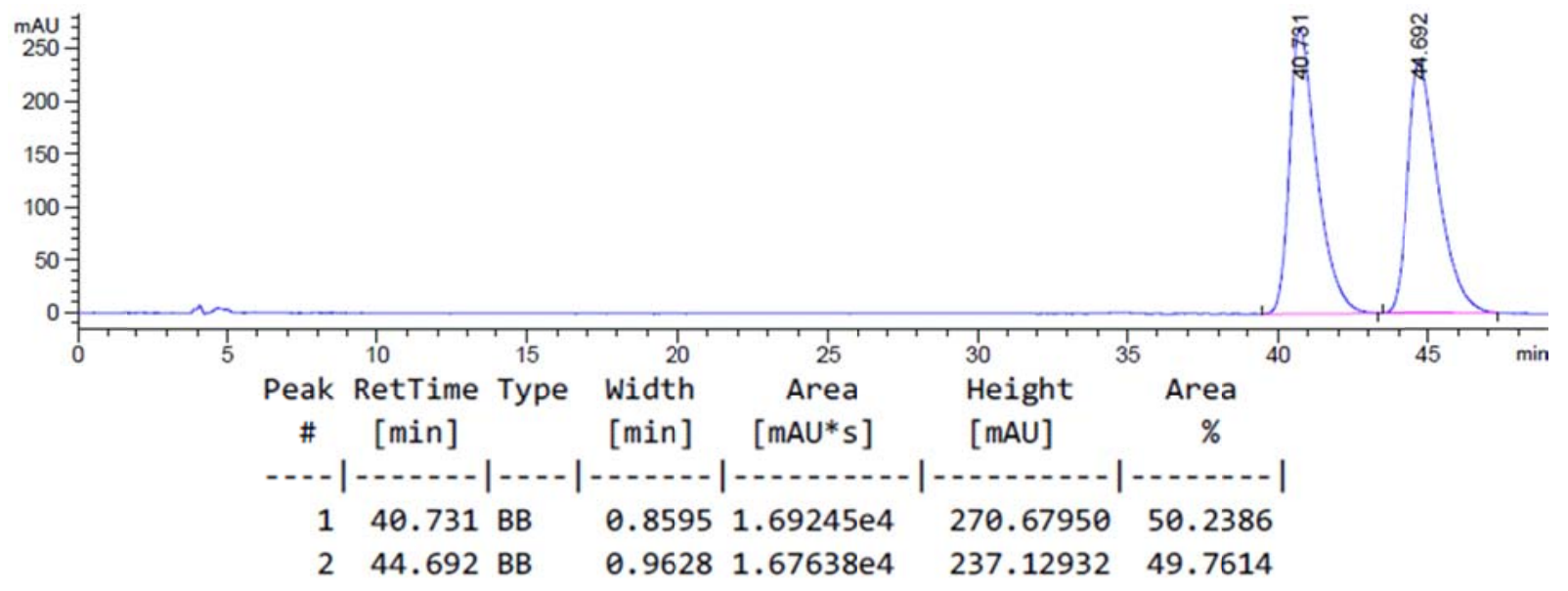

(S)-1-(4'-Nitrophenyl)ethanol [(S)-24, 80\% ee]

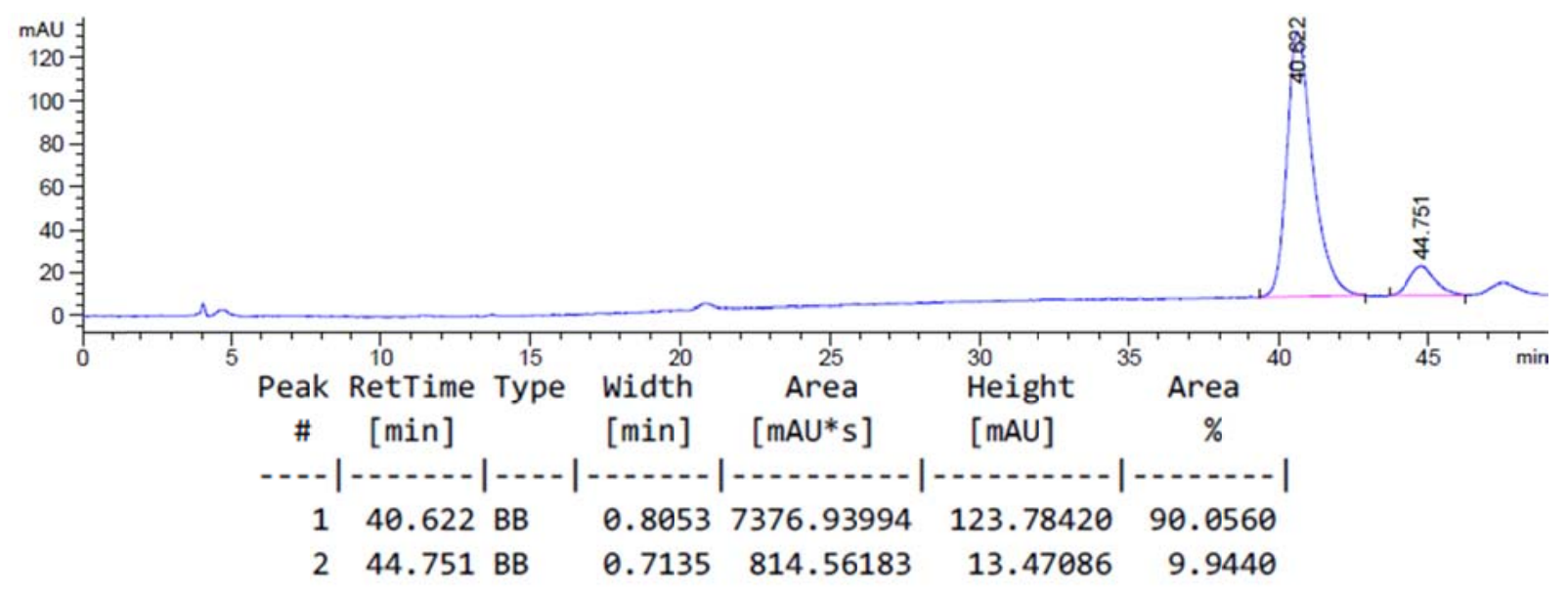




\section{(S)-Methyl-4-(1-hydroxyethyl)benzoate [(S)-25]}

The enantiomeric excess was determined by HPLC analysis on a chiral stationary phase (Daicel Chiralcel OJ-H column, column temperature $20^{\circ} \mathrm{C}$, solvent $n$-heptane:iso-propanol = $97: 3$, flow rate $0.8 \mathrm{~mL} / \mathrm{min}, \lambda=210 \mathrm{~nm}): t_{\mathrm{R}}=50.3 \mathrm{~min}$ for $(S)-25, t_{\mathrm{R}}=54.8 \mathrm{~min}$ for $(R)-25$.

rac-Methyl-4-(1-hydroxyethyl)benzoate (rac-25, synthesized according to GP 3)

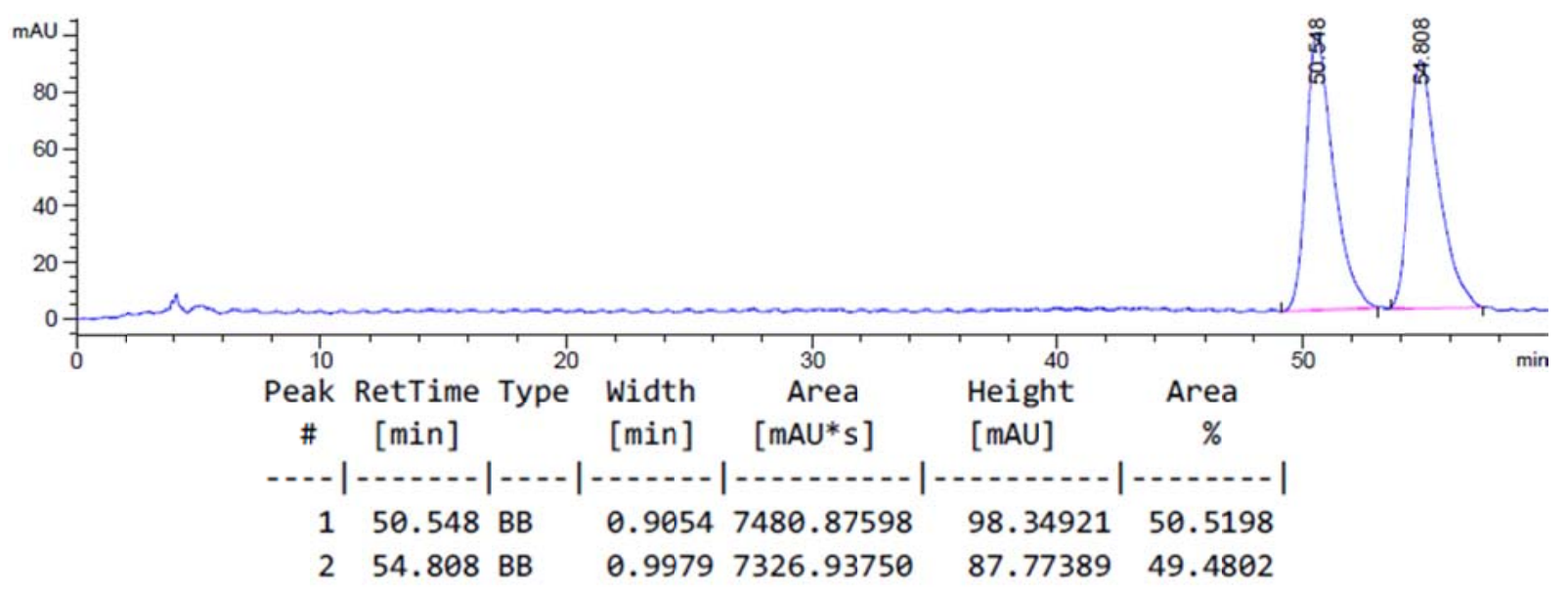

(S)-Methyl-4-(1-hydroxyethyl)benzoate [(S)-25, 98\% ee]

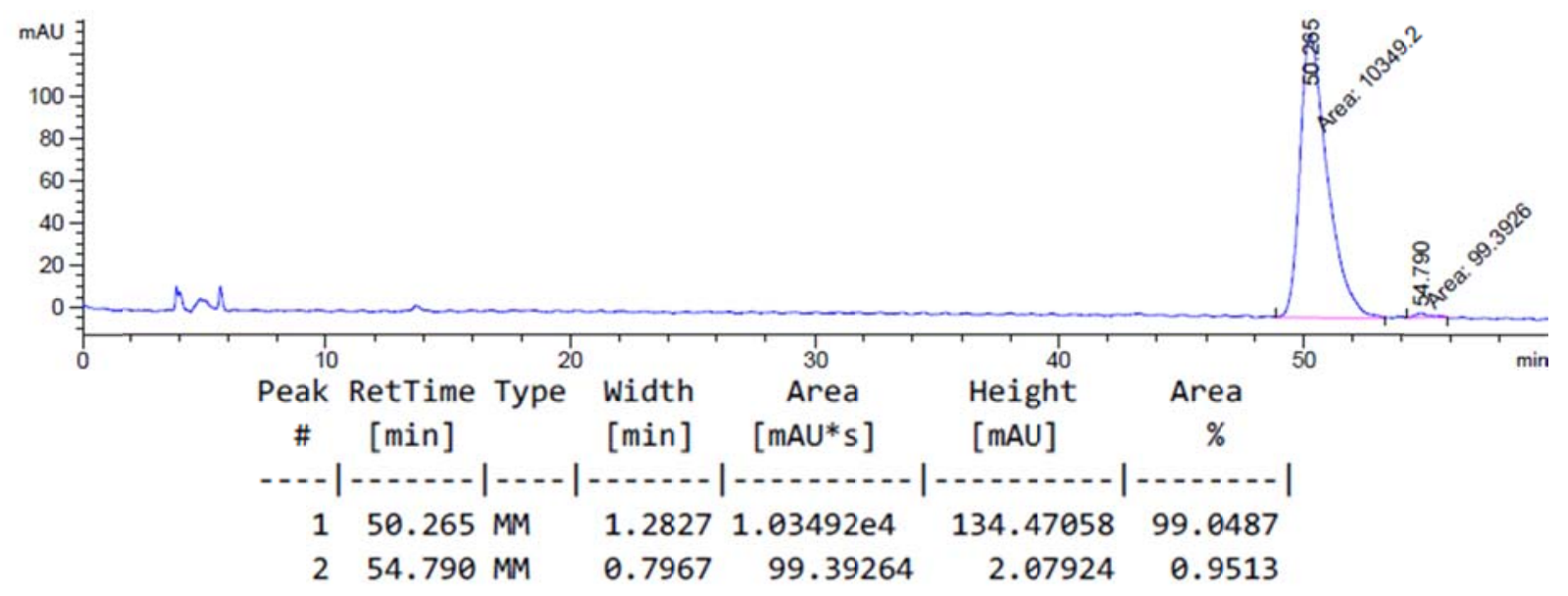




\section{(S)-1-(4'-Chlorophenyl)ethanol [(S)-26]}

The enantiomeric excess was determined by HPLC analysis on a chiral stationary phase (Daicel Chiralcel OJ-H column, column temperature $20^{\circ} \mathrm{C}$, solvent $n$-heptane:iso-propanol = 98:2, flow rate $0.8 \mathrm{~mL} / \mathrm{min}, \lambda=210 \mathrm{~nm}): t_{\mathrm{R}}=25.5 \mathrm{~min}$ for $(S)-26, t_{\mathrm{R}}=28.4 \mathrm{~min}$ for $(R)-26$.

rac-1-(4'-Chlorophenyl)ethanol (rac-26, synthesized according to GP 3)

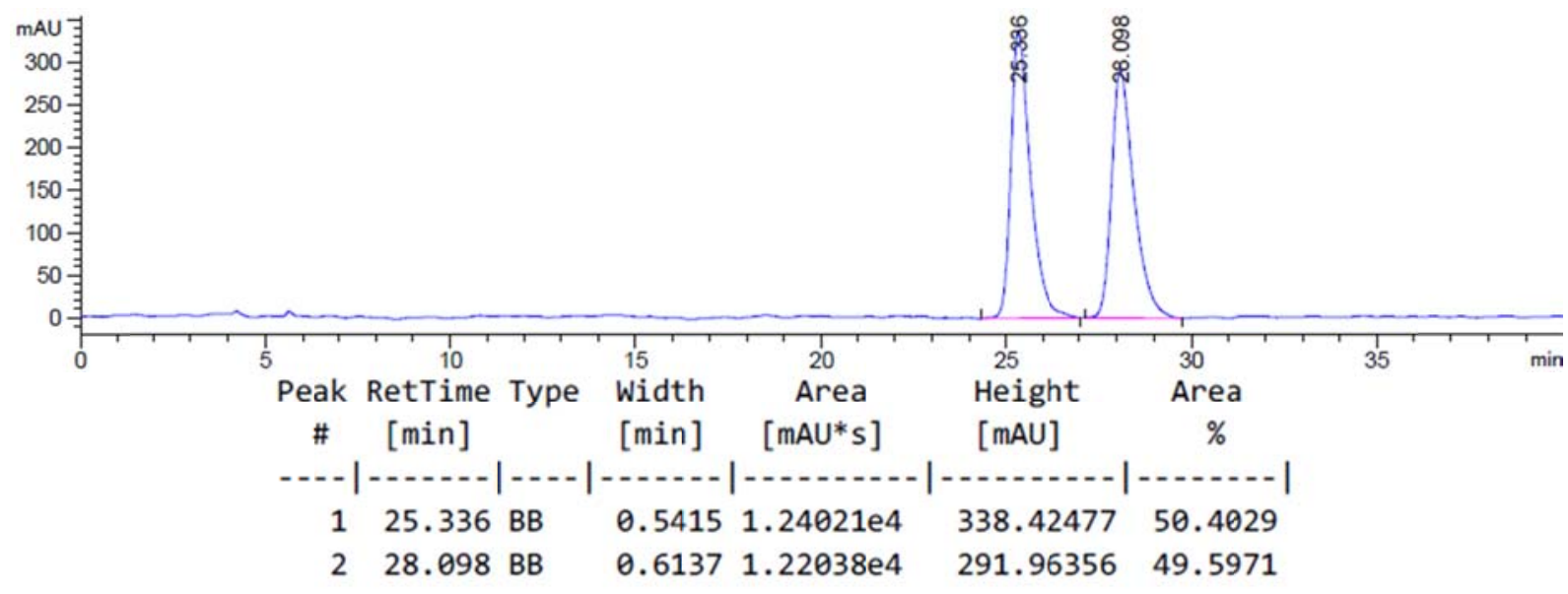

(S)-1-(4'-Chlorophenyl)ethanol [(S)-26, 95\% ee]

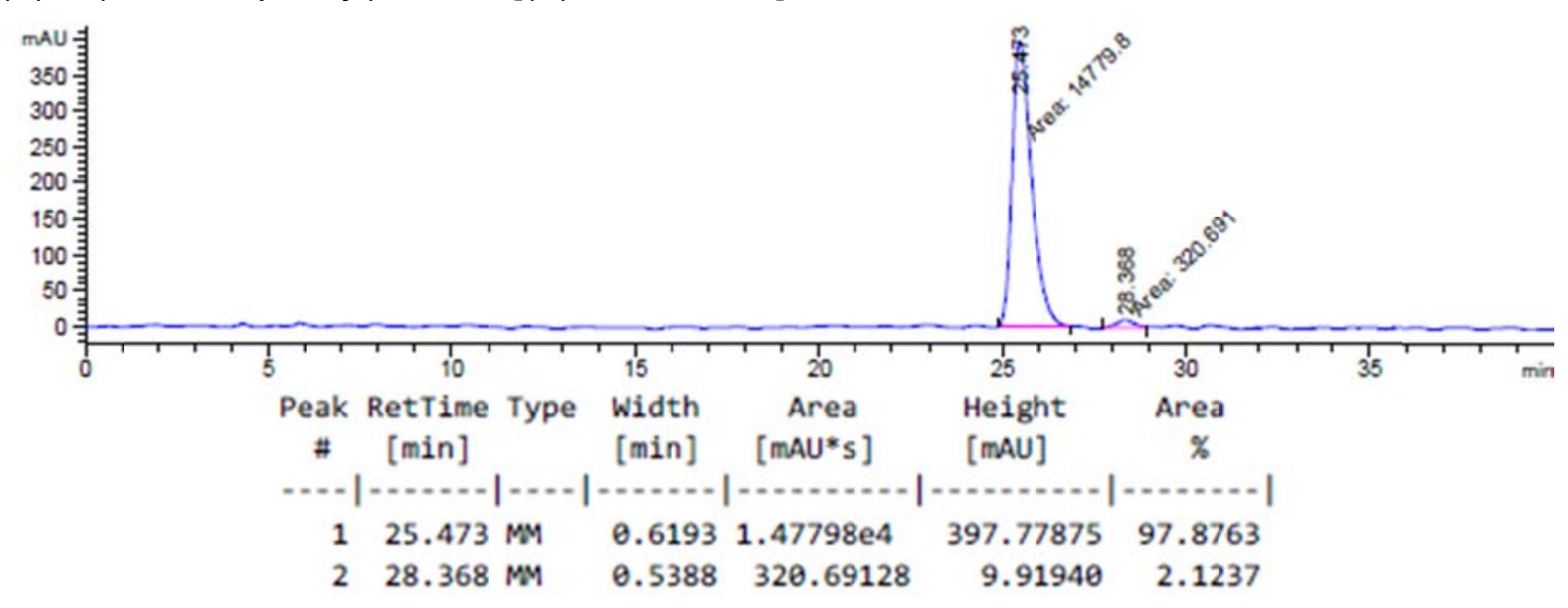




\section{(S)-1-(4'-Methylphenyl)ethanol [(S)-27]}

The enantiomeric excess was determined by HPLC analysis on a chiral stationary phase (Daicel Chiralcel OJ-H column, column temperature $20^{\circ} \mathrm{C}$, solvent $n$-heptane:iso-propanol = 98:2, flow rate $0.8 \mathrm{~mL} / \mathrm{min}, \lambda=210 \mathrm{~nm}): t_{\mathrm{R}}=26.0 \mathrm{~min}$ for $(S)-27, t_{\mathrm{R}}=30.7 \mathrm{~min}$ for $(R)-27$.

rac-1-(4'-Methylphenyl)ethanol (rac-27, purchased from TCl)

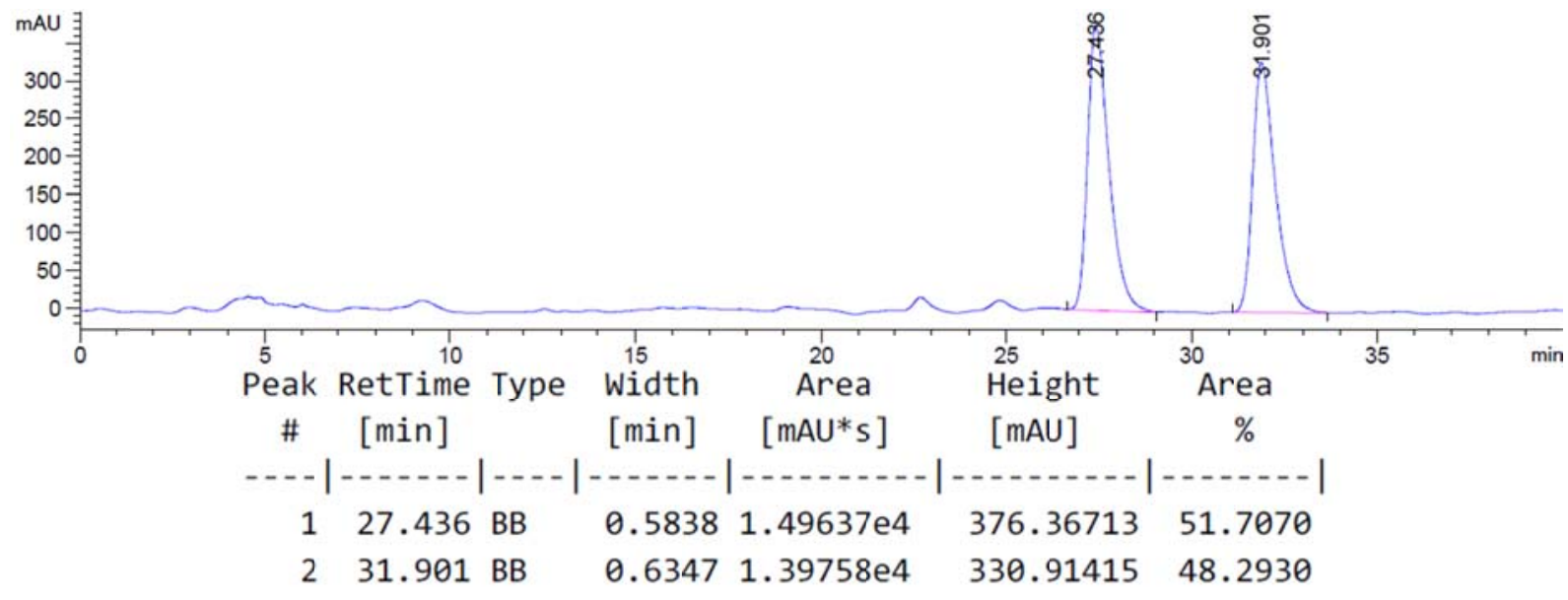

(S)-1-(4'-Methylphenyl)ethanol [(S)-27, 85\% ee]

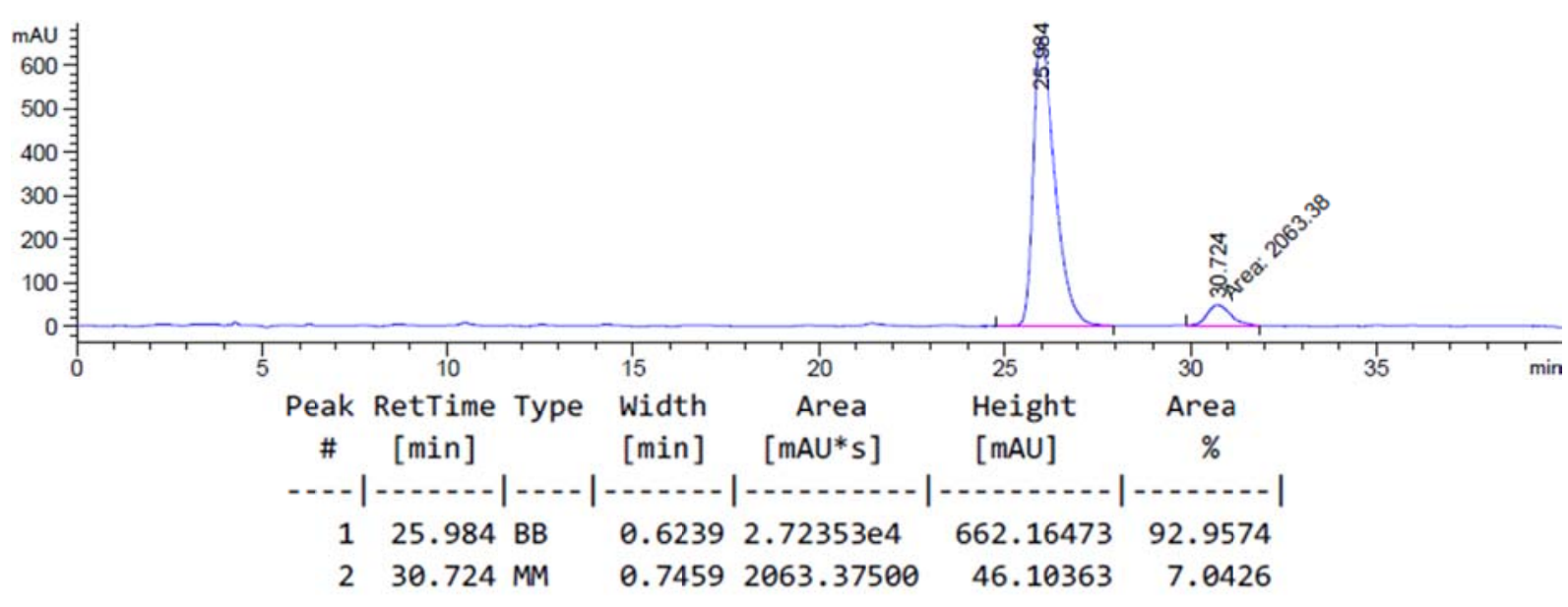




\section{(S)-1-(4-Biphenylyl)ethanol [(S)-28]}

The enantiomeric excess was determined by HPLC analysis on a chiral stationary phase (Daicel Chiralcel OD-RH column, column temperature $20^{\circ} \mathrm{C}$, solvent acetonitrile:water = 40:60, flow rate $0.4 \mathrm{~mL} / \mathrm{min}, \lambda=210 \mathrm{~nm}$ ): $t_{\mathrm{R}}=38.7 \mathrm{~min}$ for $(S)-28, t_{\mathrm{R}}=42.8 \mathrm{~min}$ for $(R)-28$.

rac-1-(4-Biphenylyl)ethanol (rac-28, synthesized according to GP 3)

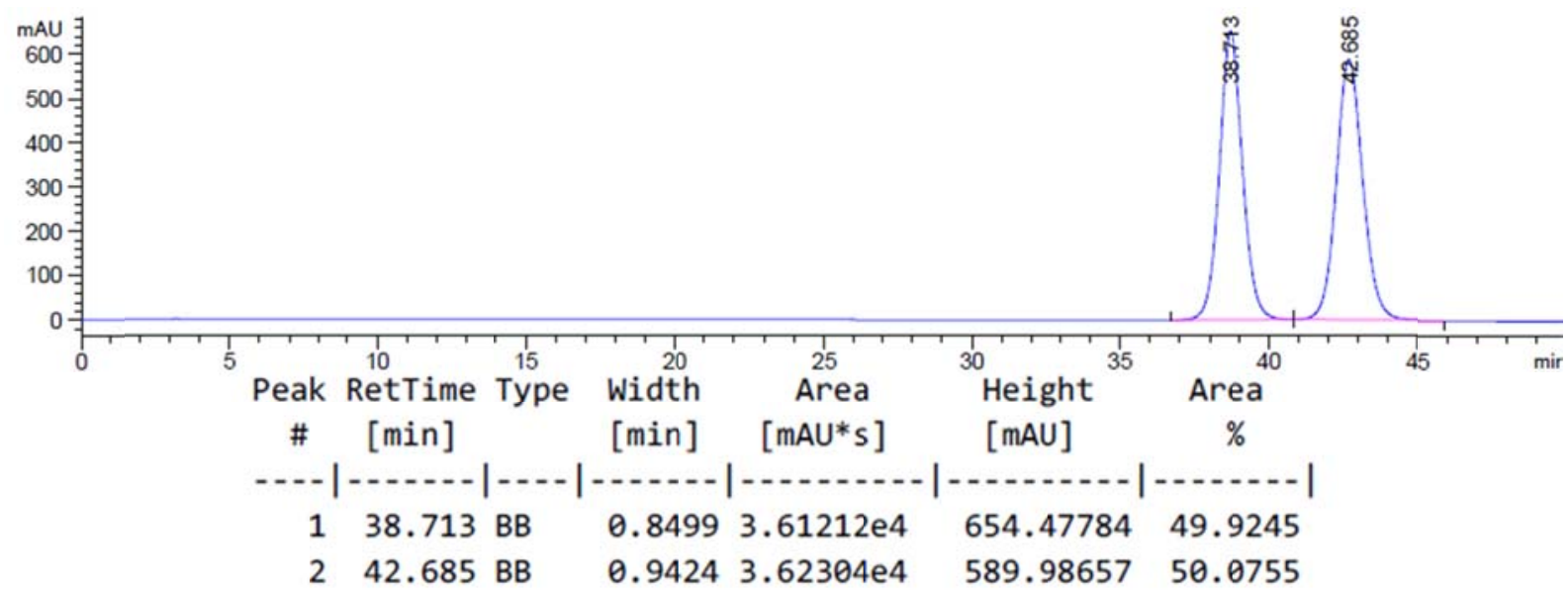

(S)-1-(4-Biphenylyl)ethanol [(S)-28, 70\% ee]

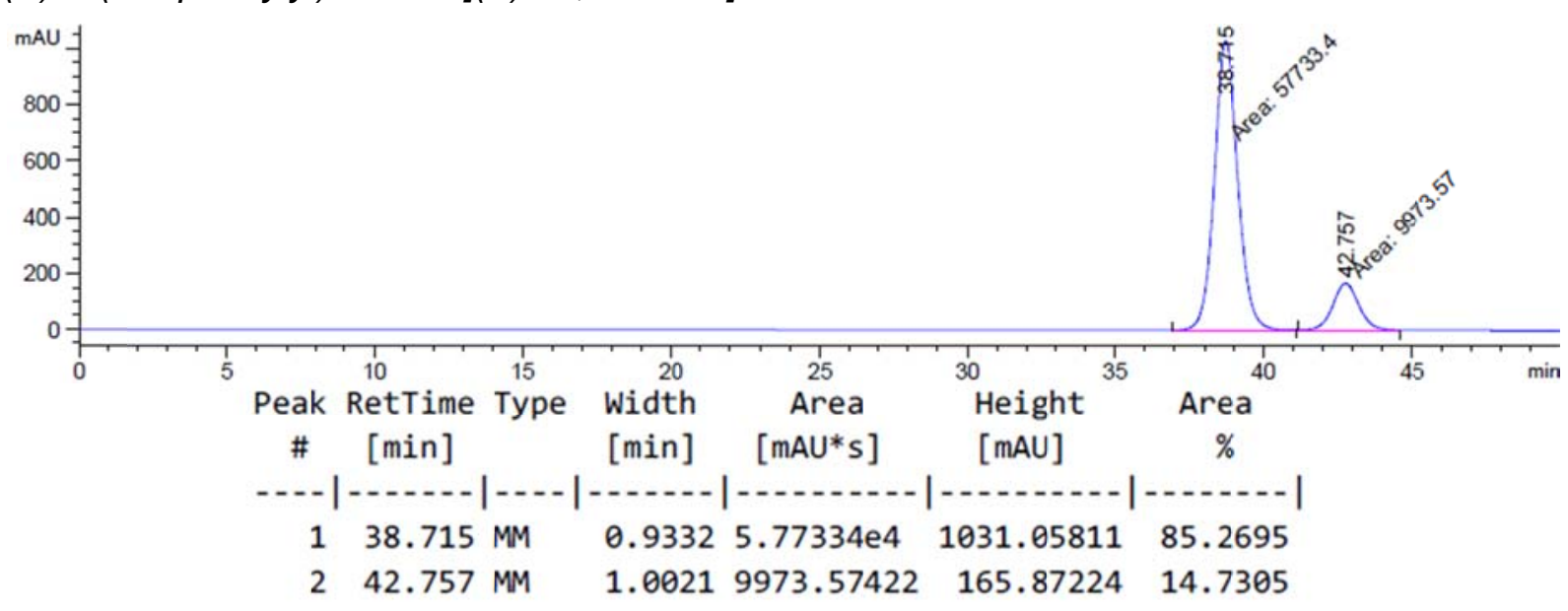




\section{(S)-1-(4'-Methoxyphenyl)ethanol [(S)-29]}

The enantiomeric excess was determined by HPLC analysis on a chiral stationary phase (Daicel Chiralcel OD-H column, column temperature $20^{\circ} \mathrm{C}$, solvent $n$-hepttane:iso-propanol = 98:2, flow rate $0.8 \mathrm{~mL} / \mathrm{min}, \lambda=210 \mathrm{~nm}): t_{\mathrm{R}}=30.6 \mathrm{~min}$ for $(R)-29, t_{\mathrm{R}}=33.6 \mathrm{~min}$ for $(S)-29$.

rac-1-(4'-Methoxyphenyl)ethanol (rac-29, synthesized according to GP 3)

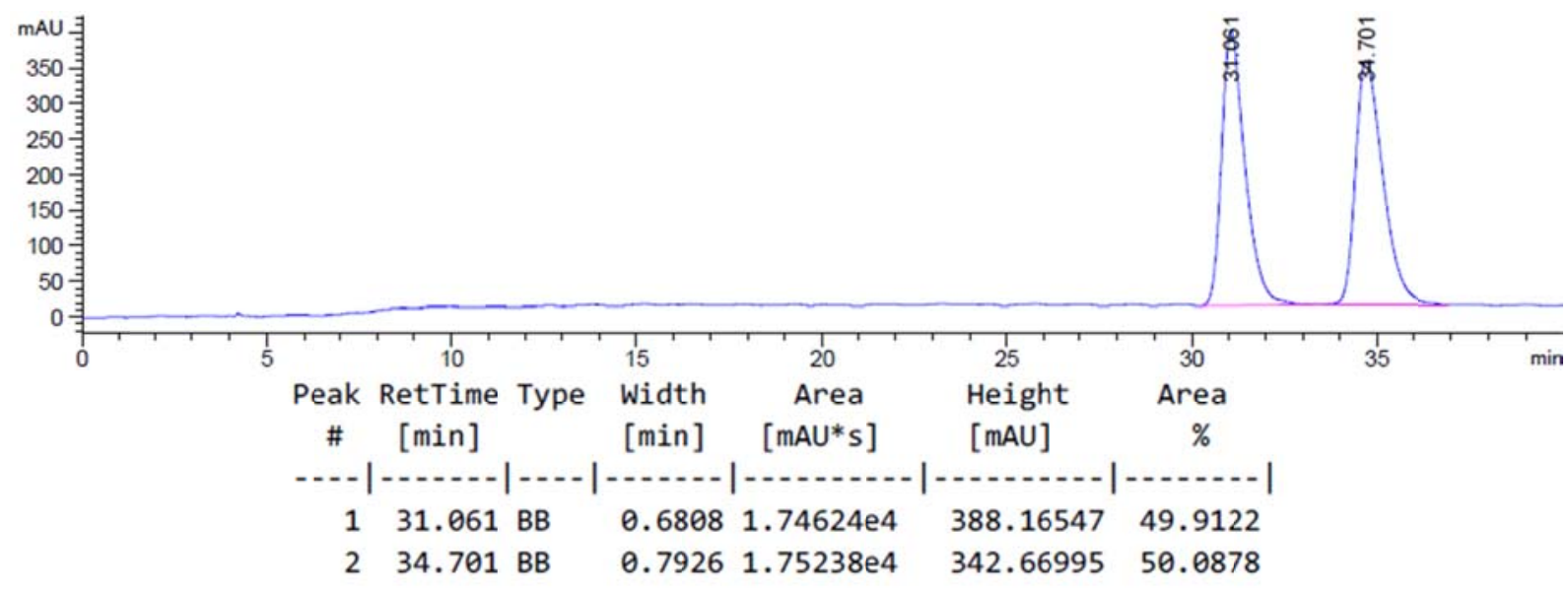

(S)-1-(4'-Methoxyphenyl)ethanol [(S)-29, 28\% ee]

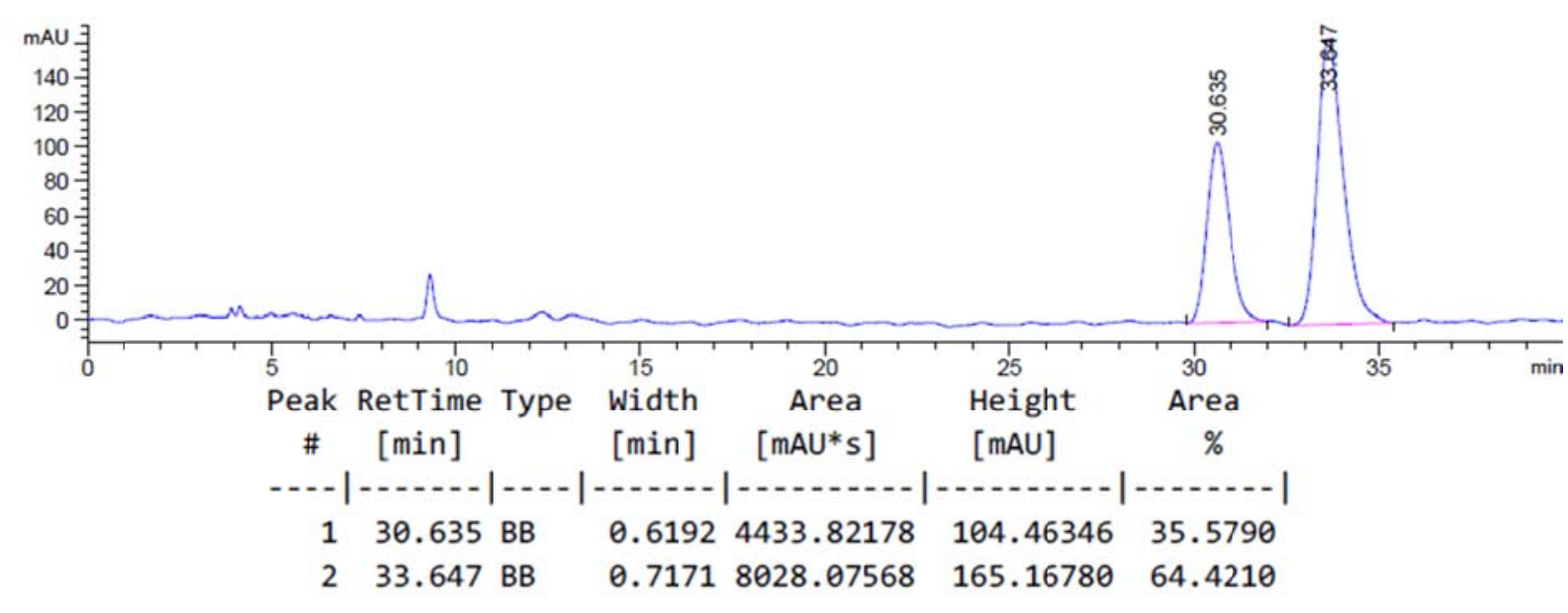




\section{(S)-1-(2',4',6'-Trimethylphenyl)ethanol [(S)-30]}

The enantiomeric excess was determined by HPLC analysis on a chiral stationary phase (Daicel Chiralcel OD-H column, column temperature $20^{\circ} \mathrm{C}$, solvent $n$-hepttane:iso-propanol = 99:1, flow rate $0.8 \mathrm{~mL} / \mathrm{min}, \lambda=210 \mathrm{~nm}): t_{\mathrm{R}}=22.9 \mathrm{~min}$ for $(S)-30, t_{\mathrm{R}}=25.9 \mathrm{~min}$ for $(R)-30$.

rac-1-(2',4',6'-Trimethylphenyl)ethanol (rac-30, synthesized according to GP 3)

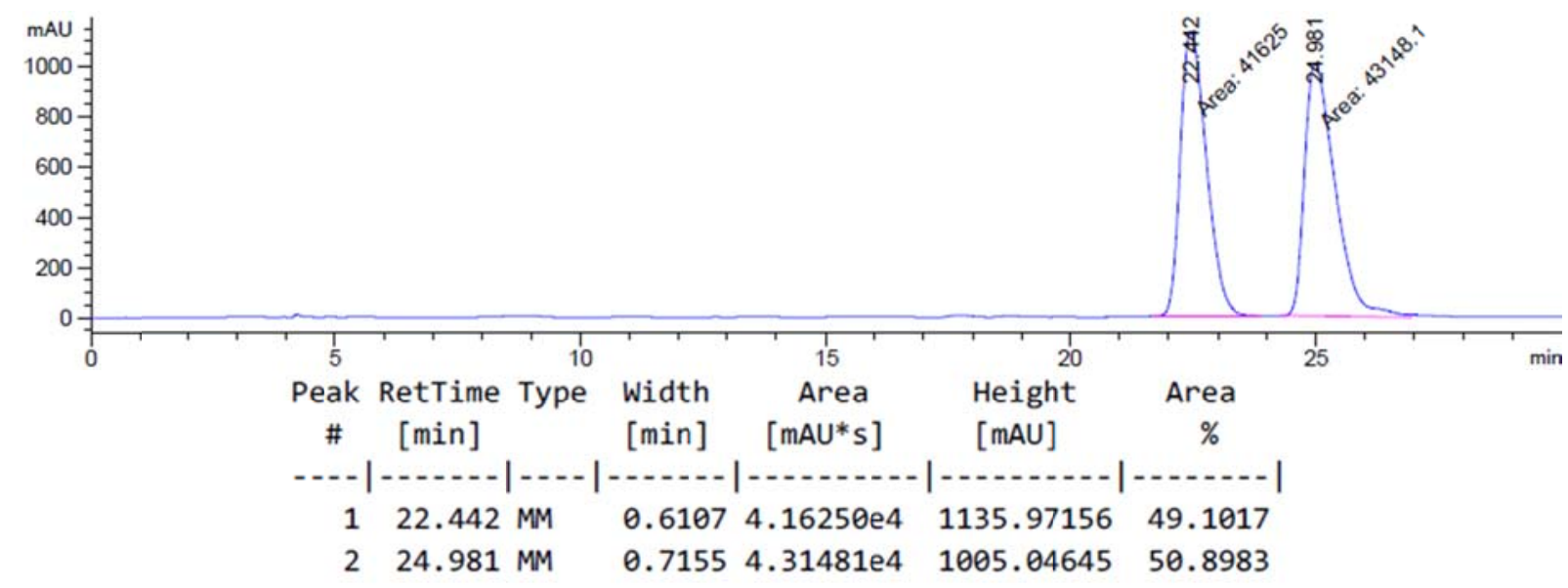

(S)-1-(2', 4', 6'-Trimethylphenyl)ethanol [(S)-30, 17\% ee]

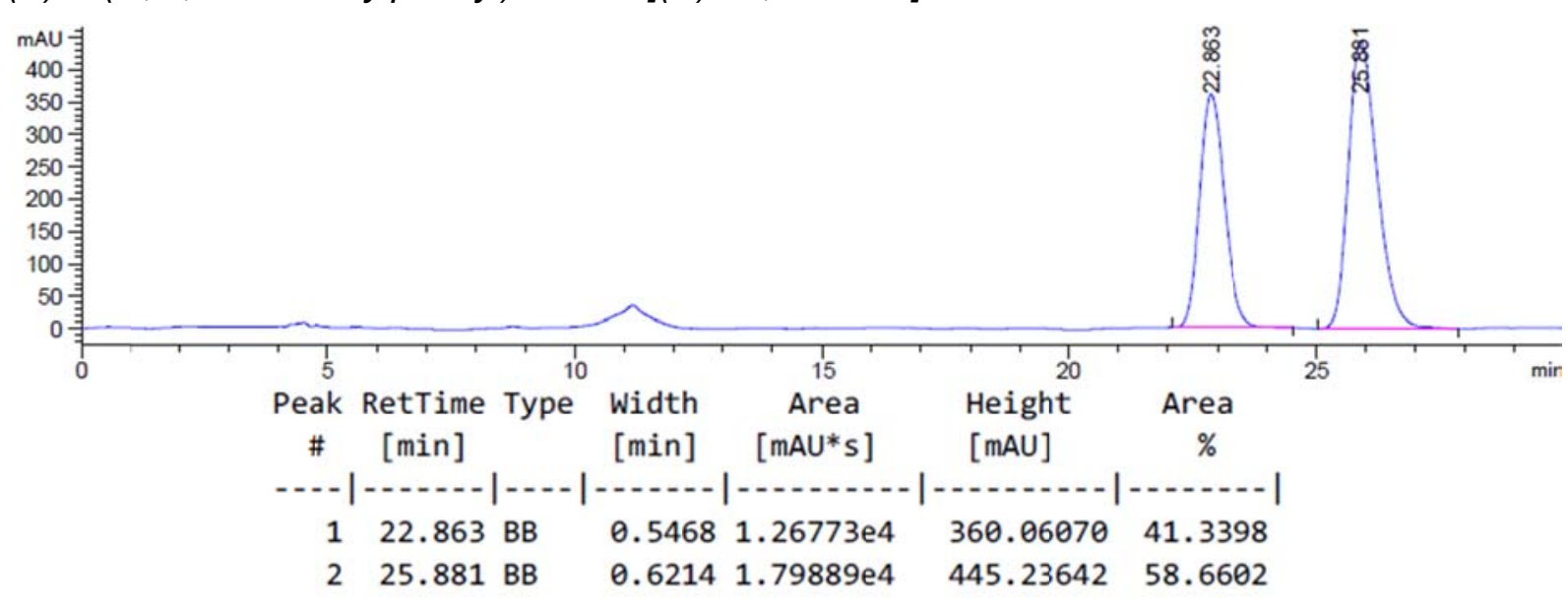




\section{(S)-1-(4'-Bromophenyl)ethanol [(S)-31]}

The enantiomeric excess was determined by HPLC analysis on a chiral stationary phase (Daicel Chiralcel OJ-H column, column temperature $20^{\circ} \mathrm{C}$, solvent $n$-heptane:iso-propanol = 98:2, flow rate $0.8 \mathrm{~mL} / \mathrm{min}, \lambda=210 \mathrm{~nm}): t_{\mathrm{R}}=28.7 \mathrm{~min}$ for $(S)-31, t_{\mathrm{R}}=32.1 \mathrm{~min}$ for $(R)-31$.

rac-1-(4'-Bromophenyl)ethanol (rac-31, synthesized according to GP 3)

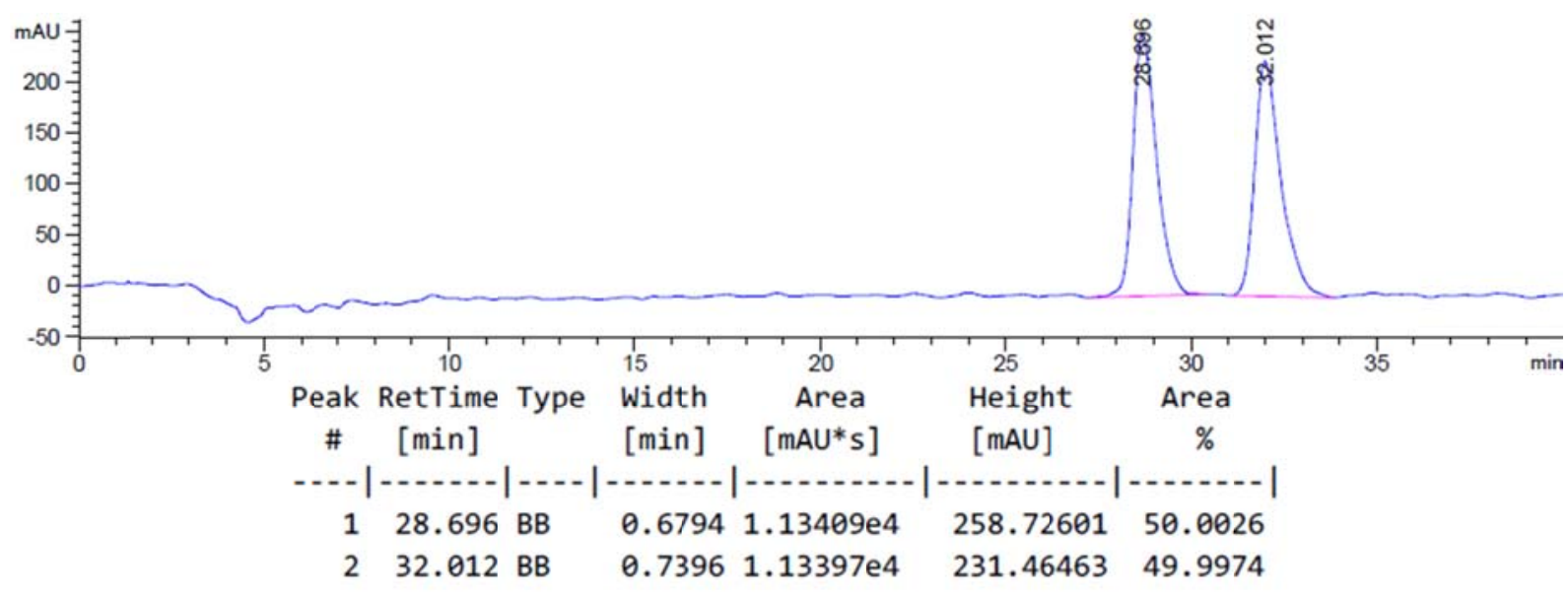

(S)-1-(4'-Bromophenyl)ethanol [(S)-31, 95\% ee]

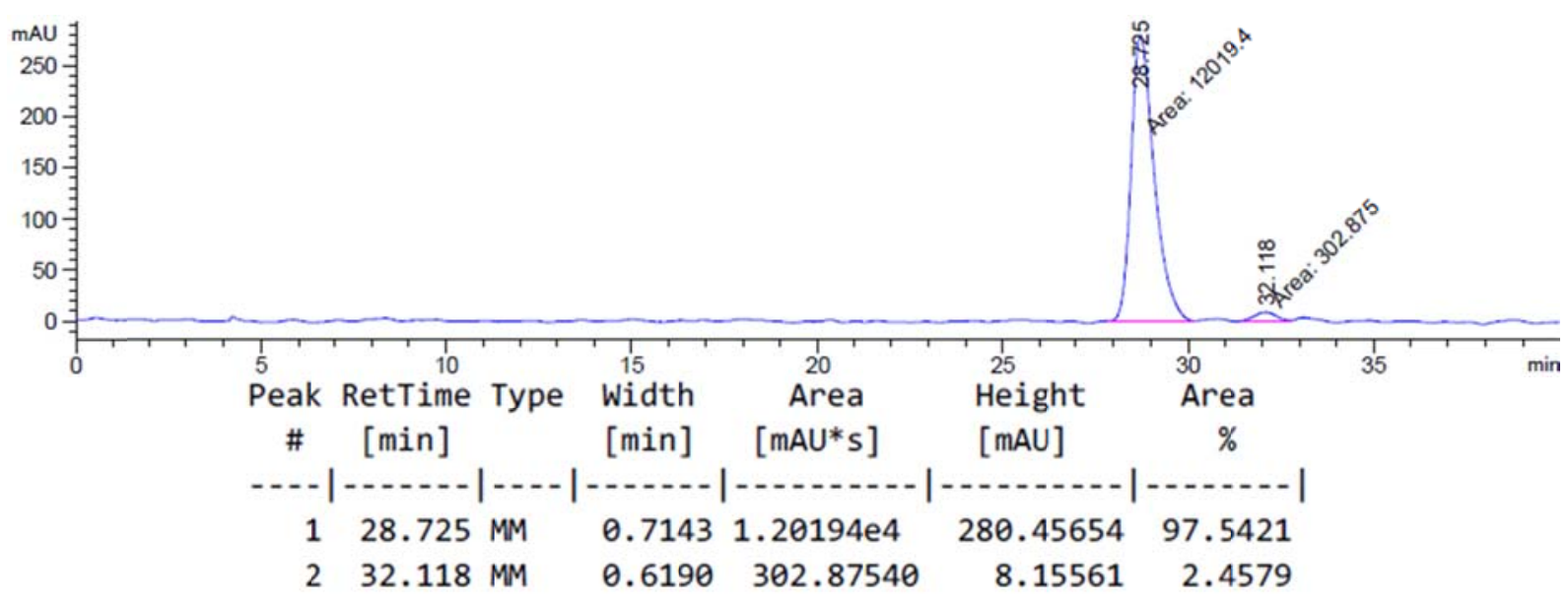




\section{(S)-1-(3'-Bromophenyl)ethanol [(S)-32]}

The enantiomeric excess was determined by HPLC analysis on a chiral stationary phase (Daicel Chiralcel OJ-H column, column temperature $20^{\circ} \mathrm{C}$, solvent $n$-heptane:iso-propanol = 98:2, flow rate $0.8 \mathrm{~mL} / \mathrm{min}, \lambda=210 \mathrm{~nm}): t_{\mathrm{R}}=25.3 \mathrm{~min}$ for $(S)-32, t_{\mathrm{R}}=30.5 \mathrm{~min}$ for $(R)-32$.

rac-1-(3'-Bromophenyl)ethanol (rac-32, synthesized according to GP 3)

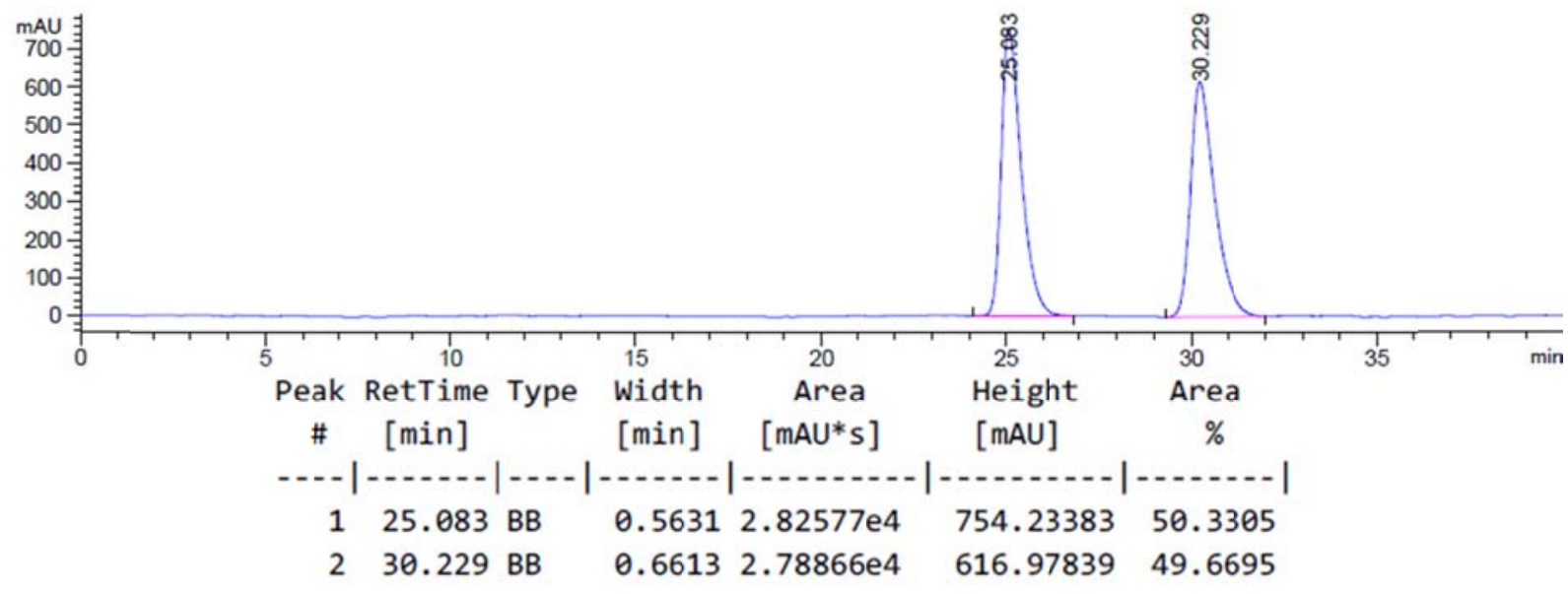

(S)-1-(3'-Bromophenyl)ethanol [(S)-32, 99\% ee]

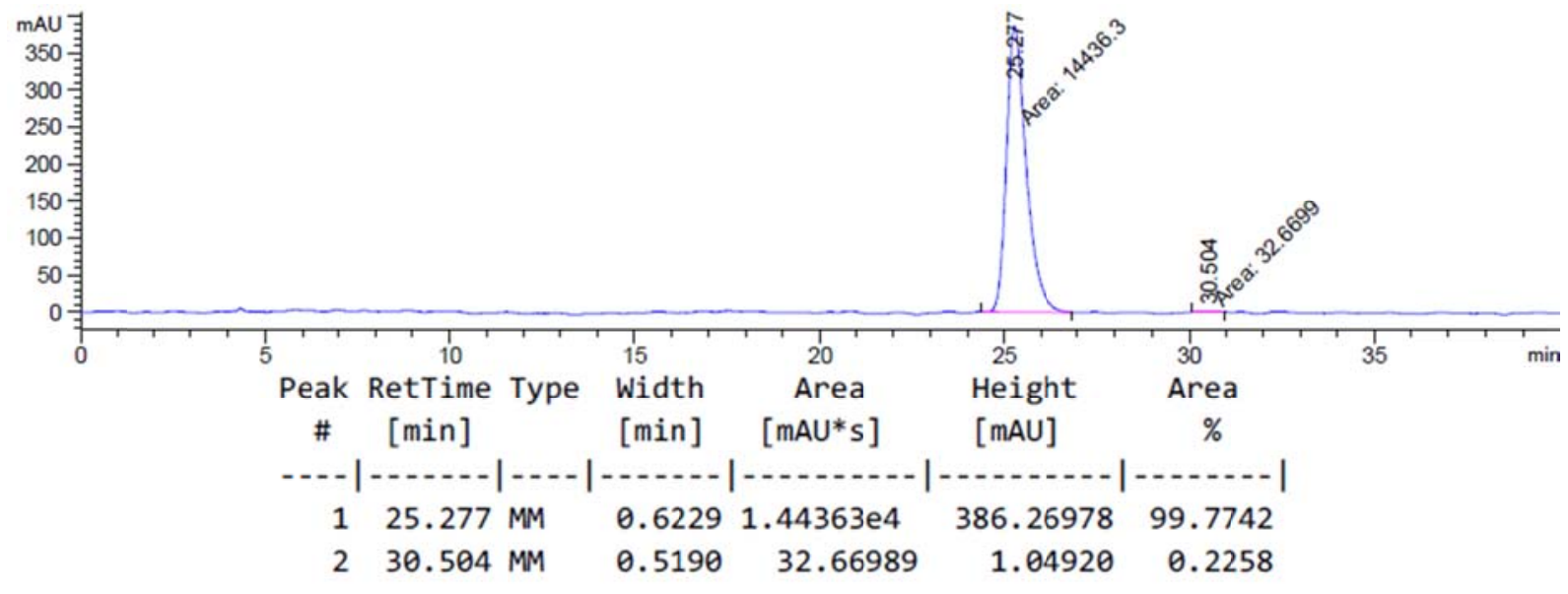




\section{(S)-1-(2'-Bromophenyl)ethanol [(S)-33]}

The enantiomeric excess was determined by HPLC analysis on a chiral stationary phase (Daicel Chiralcel OJ-H column, column temperature $20^{\circ} \mathrm{C}$, solvent $n$-heptane:iso-propanol = 98:2, flow rate $0.8 \mathrm{~mL} / \mathrm{min}, \lambda=210 \mathrm{~nm}): t_{\mathrm{R}}=20.2 \mathrm{~min}$ for $(S)-33, t_{\mathrm{R}}=22.2 \mathrm{~min}$ for $(R)-33$.

rac-1-(2'-Bromophenyl)ethanol (rac-33, synthesized according to GP 3)

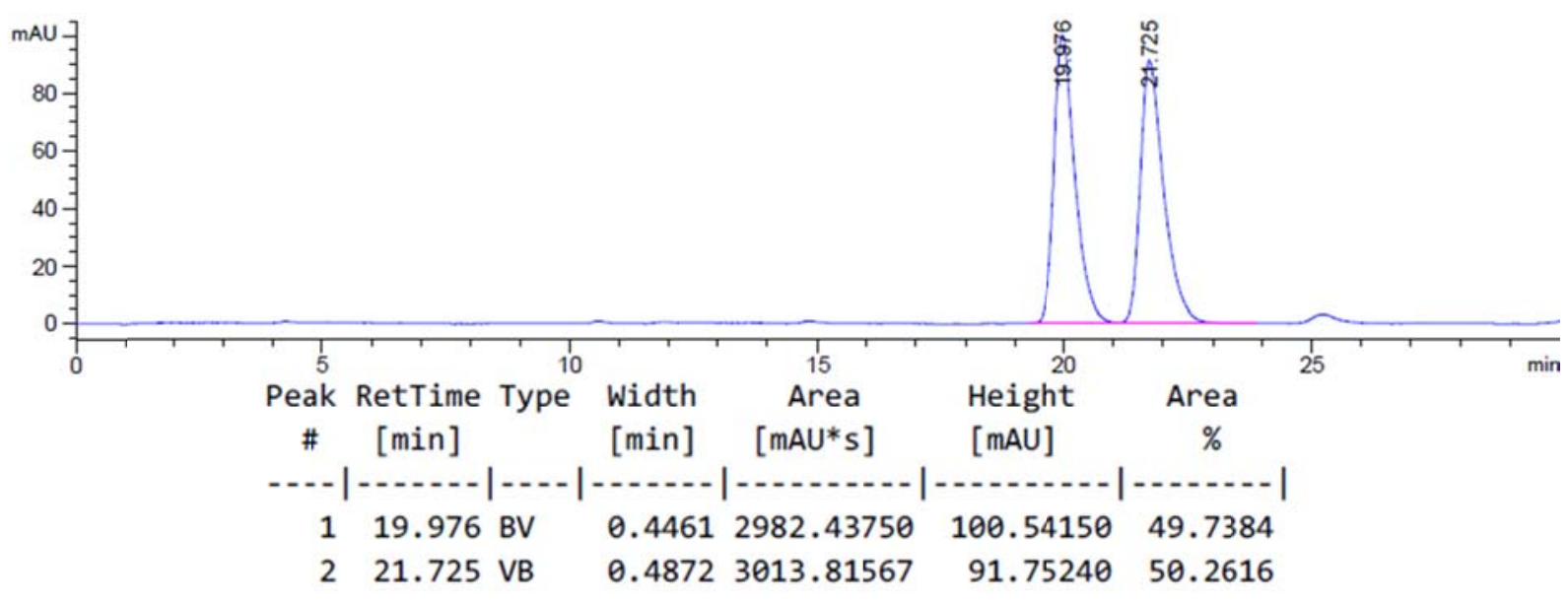

(S)-1-(2'-Bromophenyl)ethanol [(S)-33, 73\% ee]

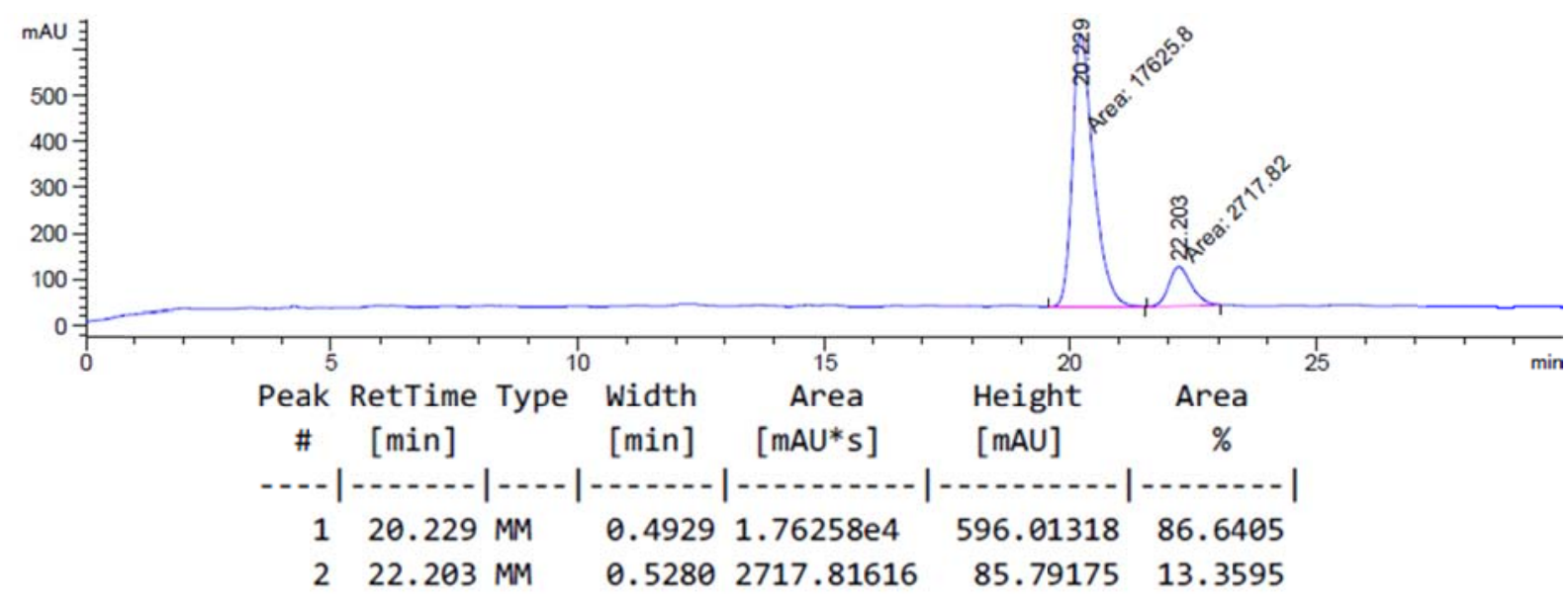




\section{(S)-1,2-Diphenylethanol [(S)-34]}

The enantiomeric excess was determined by HPLC analysis on a chiral stationary phase (Daicel Chiralcel OD-RH column, column temperature $20^{\circ} \mathrm{C}$, solvent acetonitrile:water = 40:60, flow rate $0.4 \mathrm{~mL} / \mathrm{min}, \lambda=210 \mathrm{~nm}): t_{\mathrm{R}}=6.5 \mathrm{~min}$ for $(R)-34, t_{\mathrm{R}}=11.2 \mathrm{~min}$ for $(S)-34$.

rac-1,2-Diphenylethan-1-ol (rac-34, synthesized according to GP 3)

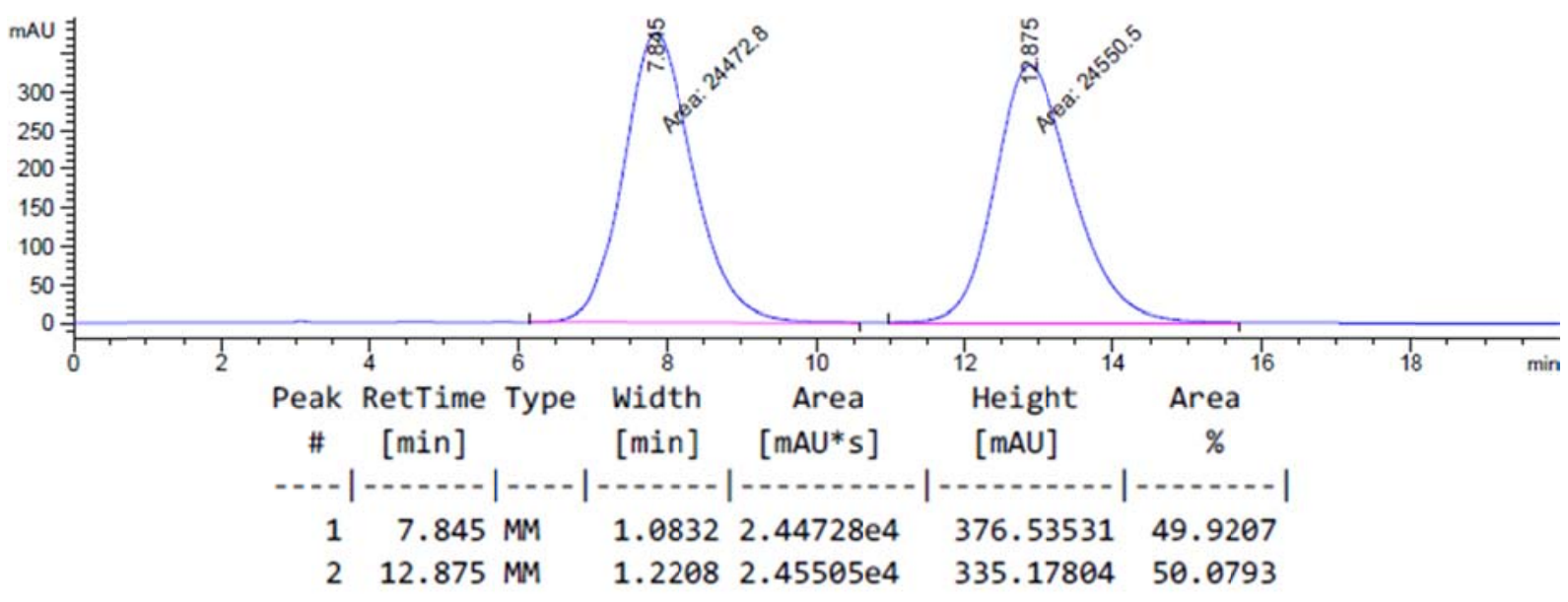

(S)-1,2-Diphenylethanol [(S)-34, 74\% ee]

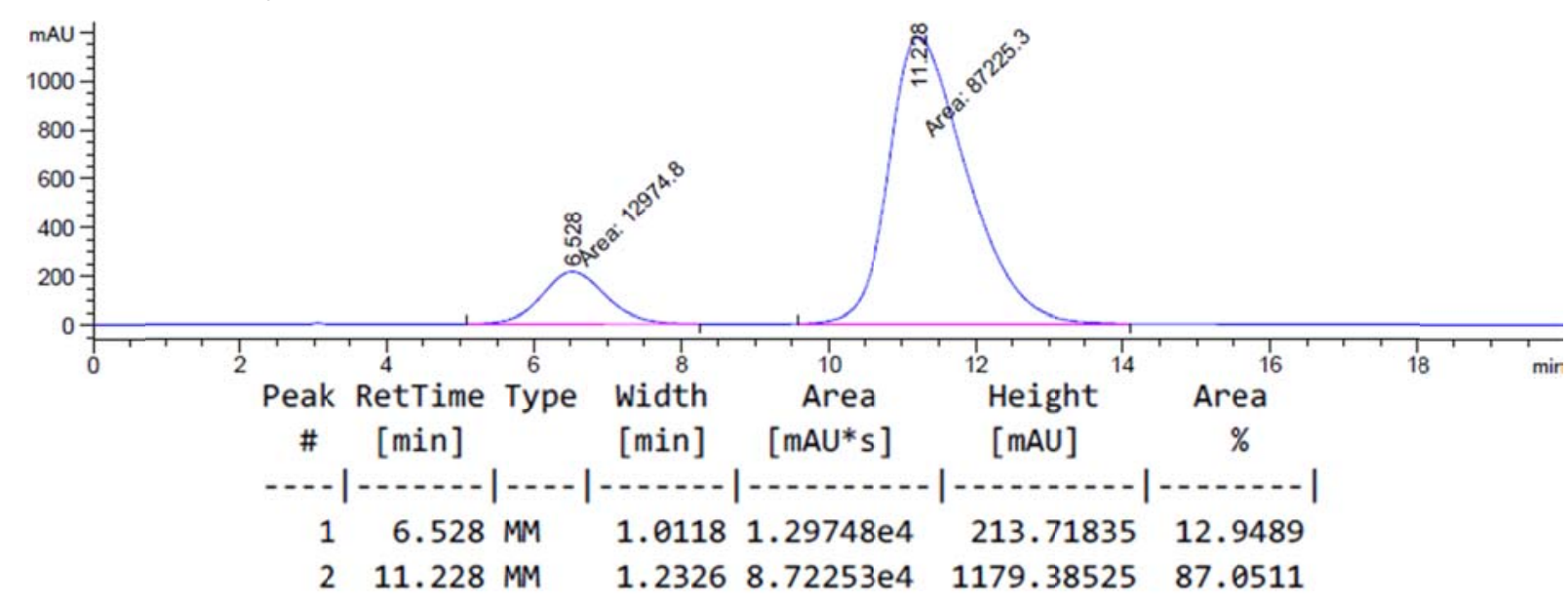




\section{(S)-Cyclohexyl(phenyl)methanol [(S)-35]}

The enantiomeric excess was determined by HPLC analysis on a chiral stationary phase (Daicel Chiralcel AD-H column, column temperature $20^{\circ} \mathrm{C}$, solvent $n$-heptane:iso-propanol = 99:1, flow rate $0.8 \mathrm{~mL} / \mathrm{min}, \lambda=210 \mathrm{~nm}): t_{\mathrm{R}}=30.8 \mathrm{~min}$ for $(S)-35, t_{\mathrm{R}}=34.1 \mathrm{~min}$ for $(R)-35$.

rac-Cyclohexyl(phenyl)methanol (rac-35, synthesized according to GP 3)

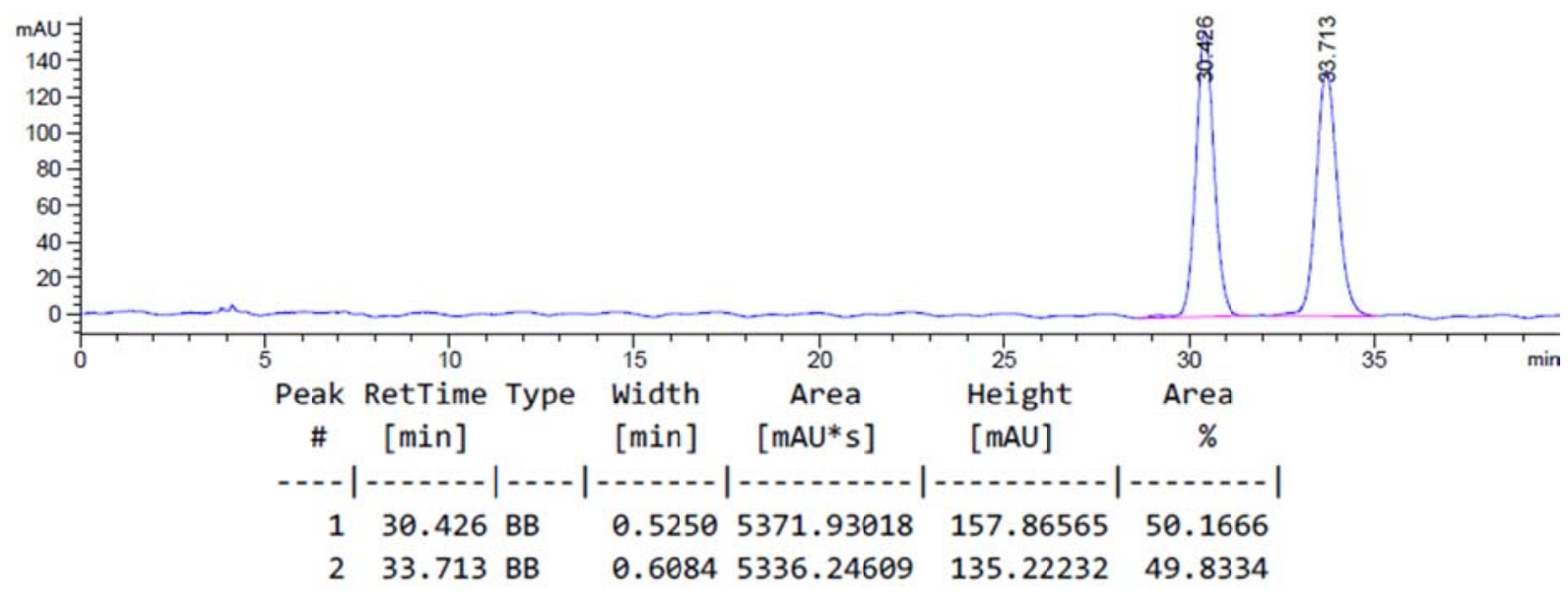

(S)-Cyclohexyl(phenyl)methanol [(S)-35, 62\% ee]

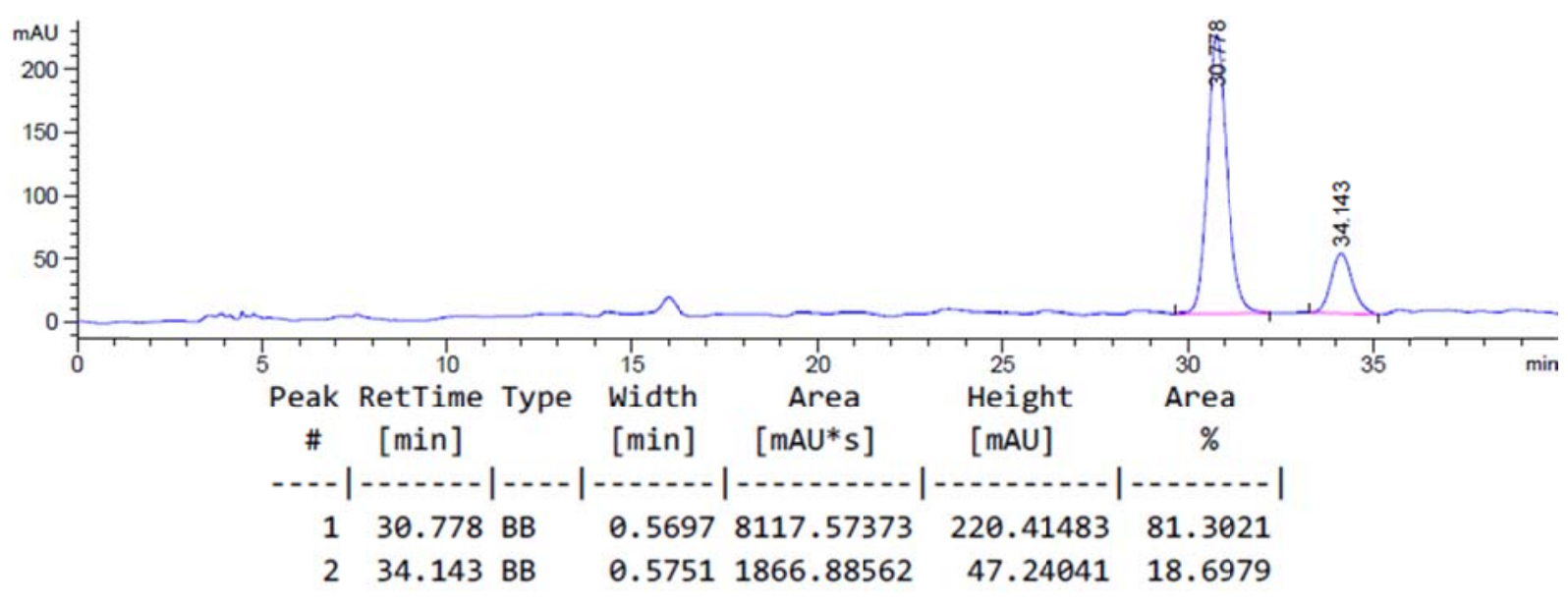




\section{(S)-1-(2'-Naphthyl)ethanol [(S)-36]}

The enantiomeric excess was determined by HPLC analysis on a chiral stationary phase (Daicel Chiralcel OJ-H column, column temperature $20^{\circ} \mathrm{C}$, solvent $n$-heptane:iso-propanol = 95:5, flow rate $0.8 \mathrm{~mL} / \mathrm{min}, \lambda=210 \mathrm{~nm}): t_{\mathrm{R}}=36.0 \mathrm{~min}$ for $(S)-36, t_{\mathrm{R}}=48.3 \mathrm{~min}$ for $(R)-36$.

rac-1-(2'-Naphthyl)ethanol (rac-36, synthesized according to GP 3)

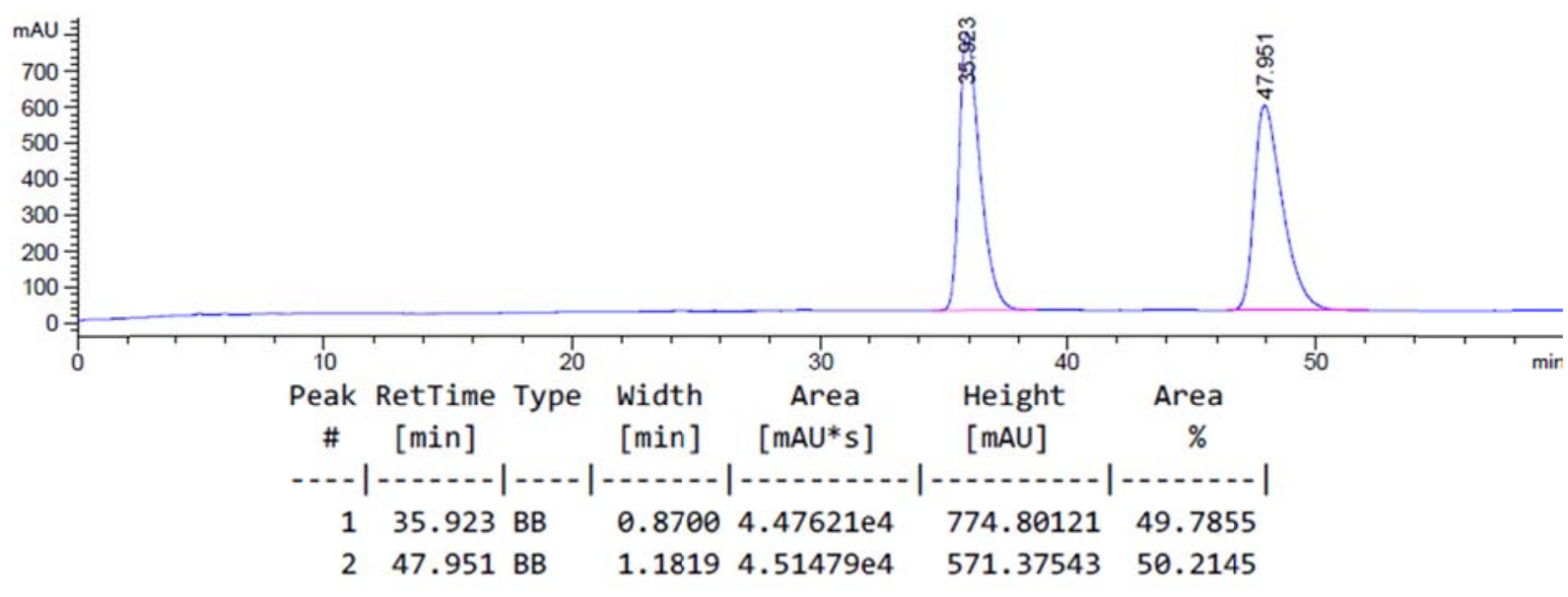

(S)-1-(2'-Naphthyl)ethanol [(S)-36, 80\% ee]

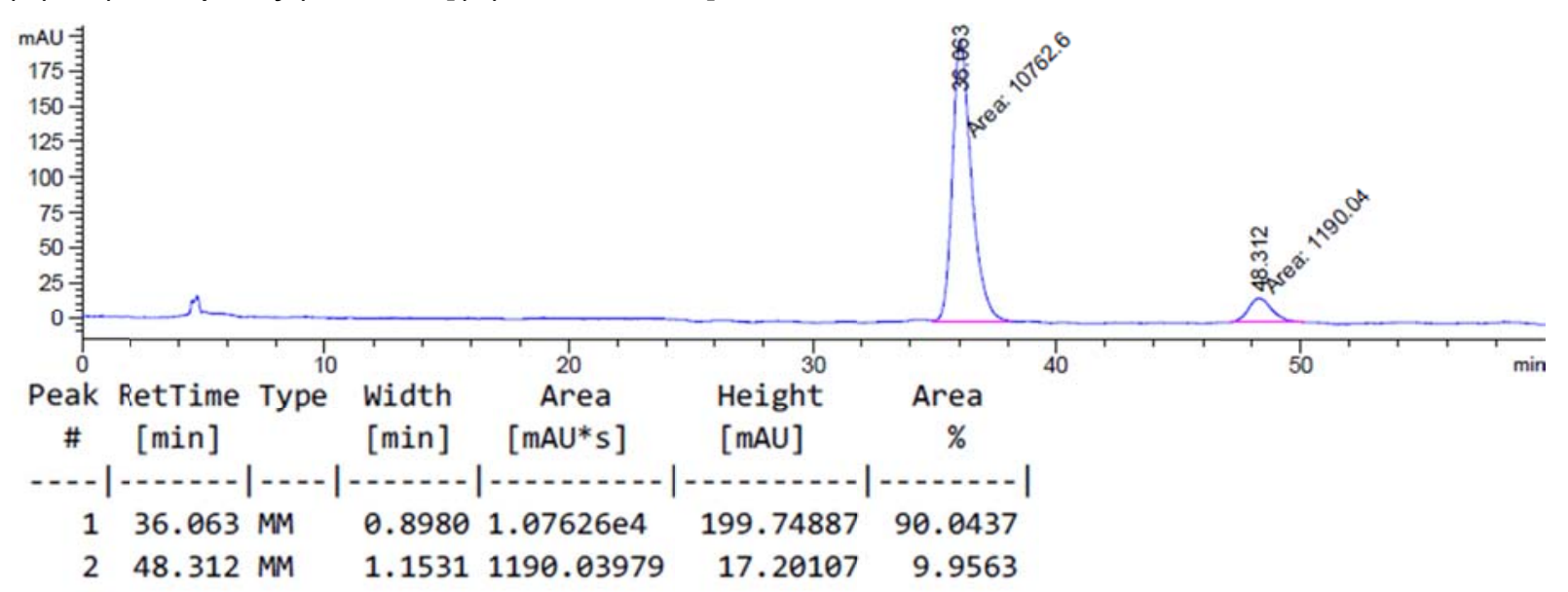




\section{(S)-4'-Bromophenyl(phenyl)methanol [(S)-37]}

The enantiomeric excess was determined by HPLC analysis on a chiral stationary phase (Daicel Chiralcel AD-H column, column temperature $20^{\circ} \mathrm{C}$, solvent $n$-heptane:iso-propanol = 95:5, flow rate $0.8 \mathrm{~mL} / \mathrm{min}, \lambda=210 \mathrm{~nm}): t_{\mathrm{R}}=18.9 \mathrm{~min}$ for $(R)-37, t_{\mathrm{R}}=21.3 \mathrm{~min}$ for $(S)-37$.

rac-4'-Bromophenyl(phenyl)methanol (rac-37, synthesized according to GP 3)

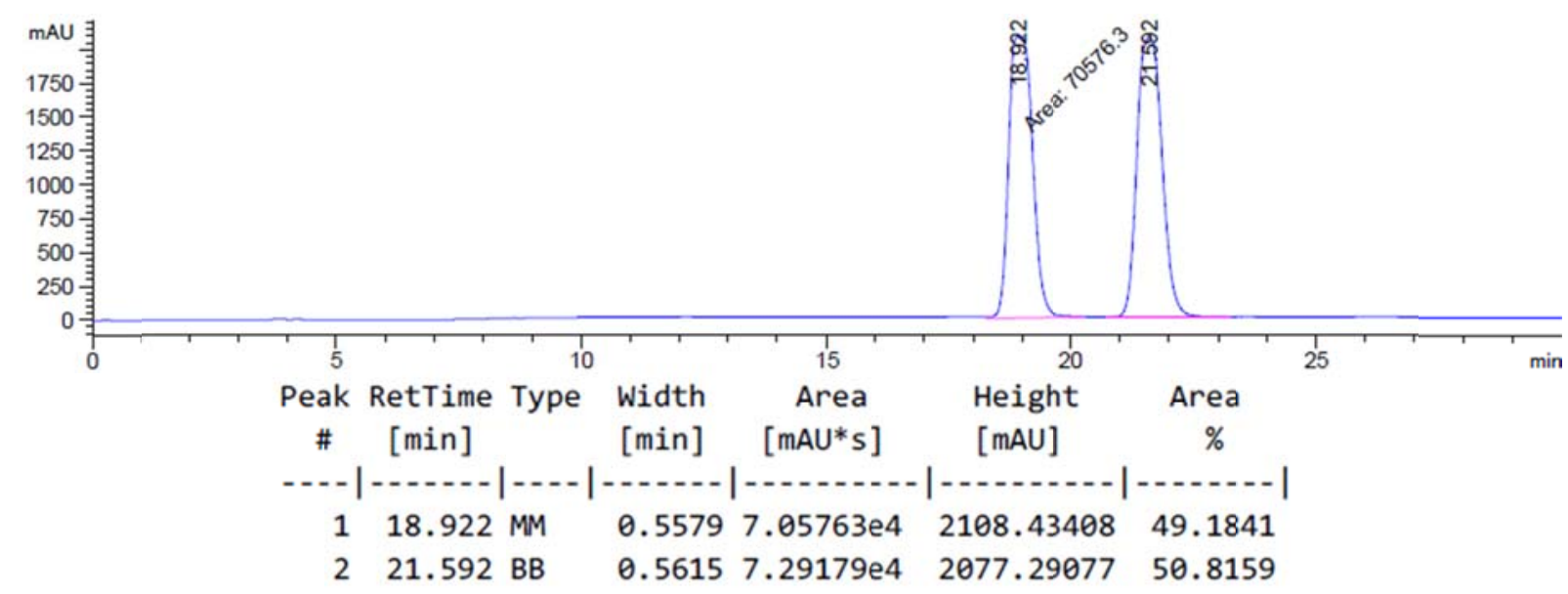

(S)-4'-Bromophenyl(phenyl)methanol [(S)-37, 14\% ee]

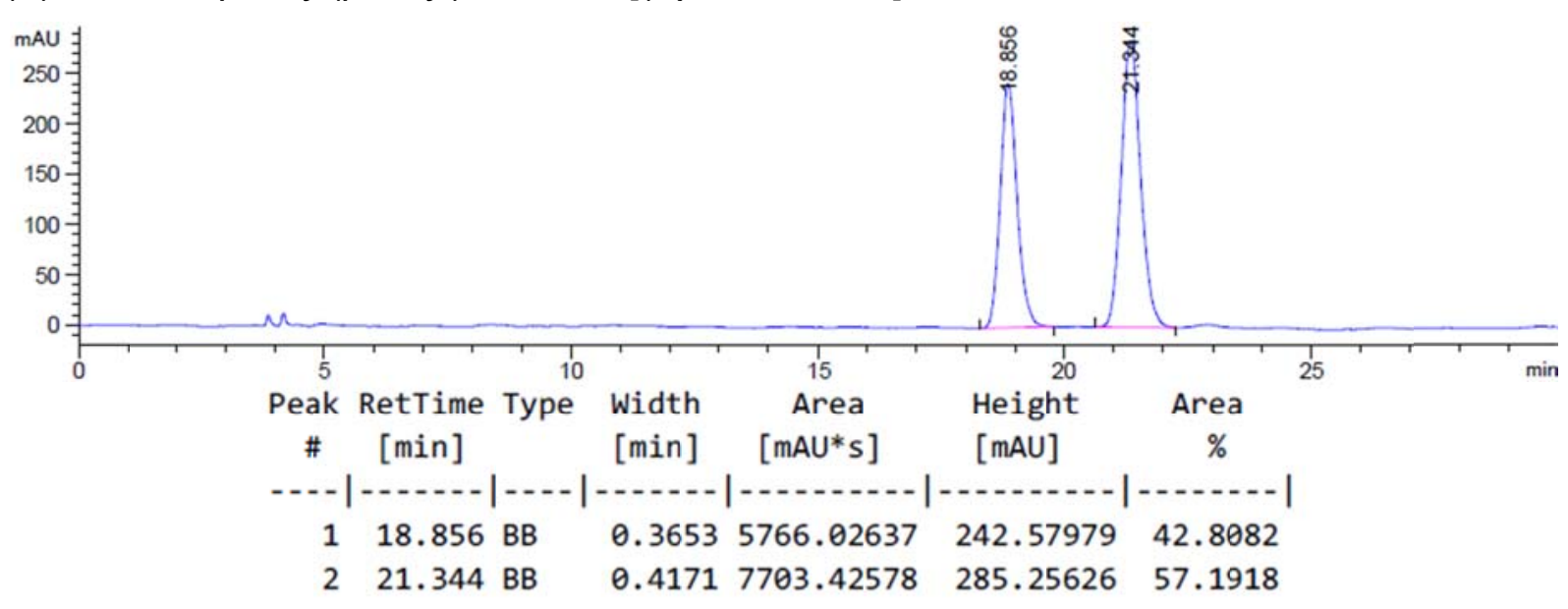




\section{References}

[S1] Hermeke, J.; Mewald, M.; Irran, E.; Oestreich, M. Organometallics 2014, 33, 50975100 .

[S2] Mewald, M.; Fröhlich, R.; Oestreich, M. Chem.-Eur. J. 2011, 17, 9406-9414.

[S3] Königs, C. D. F. ; Oestreich, M. Synthesis 2011, 2062-2065.

[S4] a) Ziegler, S. S.; Johnson, L. M.; West, R. J. Organomet. Chem. 1988, 341, 187-198. b) Fink, M. J.; Michalczyk, M. J.; Haller, K. J.; Michl, J.; West, R. Organometallics 1984, 3, 793-800. c) Söldner, M.; Šandor, M.; Schier, A.; Schmidbaur, H. Chem. Ber./Recl. 1997, 130, 1671-1676.

[S5] Agilent CrysAlis PRO, 2012, Agilent Technologies, Yarnton, UK.

[S6] Sheldrick, G. M. Acta Crystallogr., Sect. A. 1990, 46, 467-473.

[S7] Sheldrick, G. M. Acta Crystallogr., Sect. A. 2008, 64, 112-122.

[S8] Cambridge Crystallographic Data Centre:

http://www.ccdc.cam.ac.uk/Solutions/CSDSystem/Pages/Mercury.aspx.

[S9] Kantam, M. L.; Laha, S.; Yadav, J.; Likhar, P. R.; Sreedhar, B.; Jha, S.; Bhargava, S.; Udayakiran, M.; B. Jagadeesh, B. Org. Lett. 2008, 10, 2979-2982.

[S10] Rahaim, R. J.; Maleczka, R. E. Org. Lett. 2011, 13, 584-587.

[S11] Salvi, N. A.; Chattopadhyay, S. Tetrahedron 2001, 57, 2833-2839.

[S12] Ito, J.; Ujiie, S.; Nishiyama, H. Organometallics 2009, 28, 630-638.

[S13] Blakemore, P. R.; Marsden, S. P.; Vater, H. D. Org. Lett. 2006, 8, 773-776.

[S14] Driver, T. G.; Harris, J. R.; Woerpel, K. A. J. Am. Chem. Soc. 2007, 129, 3836-3837.

[S15] Glynn, D.; Shannon, J.; Woodward, S. Chem.-Eur. J. 2010, 16, 1053-1060. 